TRANSACTIONS OF THE

AMERICAN MATHEMATICAL SOCIETY

Volume 349, Number 8, August 1997, Pages 2981-3074

S 0002-9947(97)01956-9

\title{
TIME-DEPENDENT COATING FLOWS IN A STRIP, PART I: THE LINEARIZED PROBLEM
}

\author{
AVNER FRIEDMAN AND JUAN J. L. VELÁZQUEZ
}

\begin{abstract}
This work is concerned with time-dependent coating flow in a strip $0<y<1$. The Navier-Stokes equations are satisfied in the fluid region, the bottom substrate $y=0$ is moving with fixed velocity $(U, 0)$, and fluid is entering the strip through the upper boundary $y=1$. The free boundary has the form $y=f(x, t)$ for $-\infty<x<R(t)$, where $R(t)$ is the moving contact point. Our objective is to prove that if the initial data are close to those of a stationary solution (the existence of such a solution was established by the authors in an earlier paper) then the time-dependent problem has a unique solution with smooth free boundary, at least for a small time interval. In this Part I we study the linearized problem, about the stationary solution, and obtain sharp estimates for the solution and its derivatives. These estimates will be used in Part II to establish existence and uniqueness for the full nonlinear problem.
\end{abstract}

\section{Formulation of the Problem}

In this work we consider a time-dependent coating problem in the 2-dimensional strip

$$
D=\{-\infty<x<\infty, 0<y<1\} .
$$

We denote the free boundary by

$$
y=f(x, t), \quad-\infty<x<R(t)
$$

thus, $(R(t), 0)$ is the starting point of the free boundary at time $t$ (also called the contact point),

$$
\begin{aligned}
& 0<f(x, t)<1 \text { for } \quad-\infty<x<R(t), \\
& f(R(t), t)=0
\end{aligned}
$$

and the fluid region is

$$
\Omega_{f}=\{(x, t) \in D, y>f(x, t) \quad \text { if }-\infty<x<R(t)\} .
$$

Received by the editors November 17, 1995.

1991 Mathematics Subject Classification. Primary 35R35, 76D05. Secondary 35J40.

Key words and phrases. Coating flow, elliptic equations, boundary value problem, NavierStokes equations, free boundary problems.

(C) 1997 American Mathematical Society 
We seek a free boundary $y=f(x, t)$, and velocity $\vec{v}$ and pressure $p$ defined in $\Omega_{f}$, such that:

$$
\begin{array}{r}
\vec{v}_{t}+(\vec{v} \cdot \nabla) \vec{v}=\Delta \vec{v}-\nabla p \quad \text { in } \quad \Omega_{f}, \\
\nabla \cdot \vec{v}=0 \quad \text { in } \quad \Omega_{f}, \\
\vec{v}=\vec{U}+\epsilon \vec{\xi}(x, t) \quad \text { on } y=1, \\
\vec{v}=\vec{U} \quad \text { on } \quad y=0, x>R(t),
\end{array}
$$

and

$$
\begin{array}{r}
T(\vec{v}, p) \vec{n}=\sigma \kappa(f) \vec{n} \quad \text { on } \quad\{y=f\}, \\
\vec{v} \cdot \vec{n}=V_{N} \quad \text { on }\{y=f\} ;
\end{array}
$$

we also prescribe initial conditions:

$$
\vec{v}(x, y, 0)=\vec{v}_{0}(x, y), f(x, 0)=f_{0}(x) \quad \text { with } \quad R(0)=R_{0} .
$$

Here $\vec{v}=\left(v_{1}, v_{2}\right)$,

$$
T(\vec{v}, p)_{i j}=\left(\partial_{i} v_{j}+\partial_{j} v_{i}\right)-p \delta_{i j}
$$

is the stress tensor (note that $\partial_{t} v_{i}+(\vec{v} \cdot \nabla) v_{i}=\sum_{j} \partial_{j} T_{i j}$ by (1.1), (1.2)), $\vec{n}$ is the outward normal:

$$
\vec{n}=\frac{1}{\sqrt{1+f_{x}^{2}}}\left(f_{x},-1\right)
$$

$\kappa(f)$ is the curvature:

$$
\kappa(f)=-\frac{f_{x x}}{\left(1+f_{x}^{2}\right)^{3 / 2}},
$$

$V_{N}$ is the velocity of the free boundary:

$$
V_{N}=-\frac{f_{t}}{\sqrt{1+f_{x}^{2}}}
$$

$\vec{U}=(U, 0)$ is a given uniform velocity in the $x$-direction, $U>0$, and $\sigma$ is a positive constant $(1 / \sigma$ is called the capillary number).

In a previous paper [6] we considered the corresponding stationary problem and established existence and uniqueness provided $\epsilon$ is small. In the present work we shall establish existence and uniqueness for (1.1)-(1.7) for small time, say $0 \leq t<T$, provided the initial data lie in some small neighborhood of a stationary solution. This result implies that the stationary solution established in [6] is stable, at least for some small time interval.

In Part I we consider the linearization of (1.1)-(1.7) about a stationary solution with velocity close to $(U, 0)$. The computation leading to this linearized problem will be described in Part II, where the full nonlinear problem (1.1)-(1.7) will also be studied. [For the stationary problem, the linearization was derived in [5] and [6].]

The linearization involves several change of variables and, in particular, the transformation $x \rightarrow x-R(t)$ which fixes the contact point of the free boundary at the origin. Another simplification is obtained by subtracting from the solution a fixed function which satisfies the boundary conditions at $y=1$.

If we introduce the stream function $\varphi(x, y, t)$ for $(x, y) \in \bar{D}$ and the free boundary $y=f(x, t)$ for $-\infty<x \leq 0$, the linearized problem then consists of the following 
system:

$$
\begin{gathered}
\frac{\partial}{\partial t} \Delta \varphi-\Delta^{2} \varphi=\mu_{0}(x, y, t) \quad \text { in } \quad D \\
\frac{\partial \varphi}{\partial x}=\frac{\partial \varphi}{\partial y}=0 \quad \text { if } \quad y=1,-\infty<x<\infty, \\
\frac{\partial \varphi}{\partial x}=\frac{\partial \varphi}{\partial y}=0 \quad \text { if } y=0,0<x<\infty, \\
\frac{\partial f}{\partial t}+\beta_{0} \frac{\partial f}{\partial x}-\frac{\partial \varphi}{\partial x}=a_{0}(x, t) \quad \text { if } \quad y=0, x<0 \quad\left(\beta_{0}>0\right), \\
3 \frac{\partial^{3} \varphi}{\partial x^{2} \partial y}+\frac{\partial^{3} \varphi}{\partial y^{3}}+\sigma \frac{\partial^{3} f}{\partial x^{3}}-\frac{\partial^{2} \varphi}{\partial y \partial t}=b_{0}(x, t) \quad \text { if } \quad y=0, x<0, \\
\frac{\partial^{2} \varphi}{\partial x^{2}}-\frac{\partial^{2} \varphi}{\partial y^{2}}=c_{0}(x, t) \quad \text { if } \quad y=0, x<0
\end{gathered}
$$

for $t>0$, with initial conditions

$$
\varphi(x, y, 0)=\varphi_{0}(x, y), \quad f(x, 0)=f_{0}(x),
$$

where $\mu_{0}, a_{0}, b_{0}, c_{0}, \varphi_{0}, f_{0}$ are given functions.

Actually, $\beta_{0}$ is a function of $t$, but we prefer to study the problem for $\beta_{0}$ fixed, and to consider $\beta_{0}(t)$ a perturbation of the constant $\beta_{0}$ (as will be done in Part II). We have also refrained from adding the condition $f(0, t)=0$ to the linearized problem; this condition will be imposed in Part II in order to determine the unknown function $R(t)$ which does not appear in the linearized problem.

In this paper we prove that the system (1.8)-(1.14) has a global solution $(\varphi, f)$, which is unique in some sense, and we derive precise estimates for the solution in terms of the data. These estimates will be used in Part II in order to solve the full nonlinear problem (1.1)-(1.7).

For clarity we shall first ( $\S 2-12)$ consider the system (1.8)-(1.14) for zero initial data:

$$
\mu_{0}(x, y, 0)=0, a_{0}(x, 0)=0, b_{0}(x, 0)=0, c_{0}(x, 0)=0, \varphi_{0}(x, y)=0, f_{0}(x)=0 .
$$

The case of general initial data will be considered in Section 13.

The structure of the paper is as follows:

In $\S 2$ we take the Laplace transform of the linearized problem, thereby obtaining an elliptic boundary value problem for $(W, F)$ in $D$ which depends on a complex parameter $z$; here $W$ and $F$ are the Laplace transforms of $\varphi$ and $f$, respectively. As a preliminary step we derive an energy equality for this system. In $\S 3$ we prove that the system has a solution $(W, F)$ for any $z>0$ and establish uniqueness for any complex $z$ with Re $z \geq 0$. The estimates that are obtained in $\S 3$, however, are not strong enough to enable us to extend the existence results to $\operatorname{Re} z>0$.

The main subsequent effort is to derive stronger estimates that enable us to (i) establish existence of $(W, F)$ for all $z$ with $\operatorname{Re} z>0$, and (ii) take the inverse Laplace transform of $(W, F)$ in order to obtain a solution of (1.8)-(1.15). These estimates are stated in Section 5 (Lemma 5.1), and their proof is given in Sections 6-10 and 14,15 (which may be viewed as an "appendix"). The estimates are semi-local in the sense that they involve local $W^{j, p}$ norms with weights that depend on both $|z|$ and the distance $R$ to the origin. The relative size of $R$ with respect to $|z|$ plays a crucial role. The derivation of the estimates employs elliptic $W^{j, p}$ estimates (with 
weights which depend on $|z|)$ for elliptic problems for a pair $(W, F)$ with boundary conditions at $y=0$ which are either of the form

$$
W=\partial_{y} W=0 \text { for all }-\infty<x<\infty,
$$

or of the form

$$
\begin{aligned}
& z F+\beta_{0} \partial_{x} F-\partial_{x} W=a(x), \quad 3 \partial_{x}^{2} \partial_{y} W+\partial_{y}^{3} W+\sigma \partial_{x}^{3} F-\varepsilon z \partial_{y} W=b(x) ; \\
& \partial_{x}^{2} W-\partial_{y}^{2} W=c(x) \quad \text { for all }-\infty<x<\infty .
\end{aligned}
$$

These estimates are derived in Section 4 and in [7, Section 13], respectively. In the various stages of the proof of Lemma 5.1 we also make use of Liouville type theorems established in [7].

In $\S 11$ we extend the a priori estimates of Lemma 5.1, and in $\S 12$ we use these estimates together with the existence results for $z>0$ (established in §3) to extend the existence proof to all complex $z$ with $|z| \geq 1$, Re $z \geq 0$. Then, by taking the inverse Laplace transform, we obtain a unique solution to (1.8)-(1.15) satisfying appropriate estimates.

In Section 13 we extend the results of the paper to the case of general initial conditions.

\section{The Laplace transform of the Linearized PRoblem}

We introduce the Laplace transform

$$
k(t) \rightarrow \int_{0}^{\infty} e^{-z t} k(t) d t
$$

and denote by $W, F, \mu, a, b, c$ the Laplace transforms of $\varphi, f, \mu_{0}, a_{0}, b_{0}, c_{0}$, respectively. The functions $W, F$ then satisfy:

$$
\begin{array}{r}
z \Delta W-\Delta^{2} W=\mu(x, y, z) \quad \text { in } \quad D \\
\frac{\partial W}{\partial x}=\frac{\partial W}{\partial y}=0 \quad \text { if } \quad y=1,-\infty<x<\infty \\
W=\frac{\partial W}{\partial y}=0 \quad \text { if } \quad y=0, x>0 \\
z F+\beta_{0} \frac{\partial F}{\partial x}-\frac{\partial W}{\partial x}=a(x, z) \quad \text { if } \quad y=0, x<0 \\
3 \frac{\partial^{3} W}{\partial x^{2} \partial y}+\frac{\partial^{3} W}{\partial y^{3}}+\sigma \frac{\partial^{3} F}{\partial x^{3}}-z \frac{\partial W}{\partial y}=b(x, z) \quad \text { if } \quad y=0, x<0 \\
\frac{\partial^{2} W}{\partial x^{2}}-\frac{\partial^{2} W}{\partial y^{2}}=c(x, z) \quad \text { if } \quad y=0, x<0
\end{array}
$$

We shall presently study (2.1)-(2.6) under the assumptions that, for some small $\epsilon_{0}>0$,

$$
\begin{gathered}
a(x, z)=0, b(x, z)=0, c(x, z)=0 \quad \text { if } \quad x>-\epsilon_{0} \quad \text { or } \quad x<-\frac{1}{\epsilon_{0}} \\
\mu(x, y, z)=0 \quad \text { if } \quad|x|>\frac{1}{\epsilon_{0}} .
\end{gathered}
$$


We first reduce the system to one for which $a \equiv b \equiv c \equiv 0$. To do this we construct functions $H(x, y, z), B(x, z)$ satisfying the same boundary conditions as $W, F$ and supported in $\left\{-1 / \epsilon_{1} \leq x \leq-\epsilon_{1}\right\}$, for some $0<\epsilon_{1}<\epsilon_{0}$.

Suppressing the parameter $z$, we choose $H$ to have the form

$$
H(x, y)=\gamma(x)+\frac{\lambda(x)}{2} y^{2}+\frac{\delta(x) y^{3}}{y} .
$$

Then we need to require that

$$
\begin{aligned}
z B(x)+\beta_{0} \frac{\partial B}{\partial x}-\frac{\partial \gamma}{\partial x} & =a(x), \\
\delta(x)+\sigma \frac{\partial^{3} B}{\partial x^{3}} & =b(x), \\
\frac{\partial^{2} \gamma}{\partial x^{2}}-\lambda(x) & =c(x) .
\end{aligned}
$$

We choose $B(x)$ to be a function supported in $\left\{-1 / \epsilon_{1} \leq x \leq-\epsilon_{1}\right\}$, such that

$$
\int_{-\infty}^{0} z B(x) d x=\int_{-\infty}^{0} a(x) d x,
$$

and set

$$
\bar{a}(x)=z B(x)+\beta_{0} \frac{\partial B}{\partial x} .
$$

Then $\bar{a}$ is supported in $\left\{-1 / \epsilon_{1} \leq x \leq-\epsilon_{1}\right\}$ and, by (2.13),

$$
\int_{-\infty}^{0} \bar{a}(x) d x=\int_{-\infty}^{0} a(x) d x .
$$

Equation (2.10) is satisfied if $\partial \gamma / \partial x=\bar{a}-a$, i.e., if

$$
\gamma(x)=\int_{x}^{0}(a-\bar{a}) d x
$$

observe that the support of $\gamma(x)$ is contained in $\left\{-1 / \epsilon_{1} \leq x \leq-\epsilon_{1}\right\}$. Finally, if we define $\delta(x)$ and $\lambda(x)$ directly by (2.11) and (2.12), then these functions vanish if $x>-\epsilon_{1}$ and if $x<-1 / \epsilon_{1}$.

Let $\zeta$ be a cutoff function which equals 1 in an $\left(\epsilon_{1} / 2\right)$-neighborhood of the interval $\left\{y=0,-1 / \epsilon_{1}<x<-\epsilon_{1}\right\}$ and vanishes outside a $\left(3 \epsilon_{1} / 4\right)$-neighborhood of this interval, and define

$$
\hat{W}=W-\zeta H, \hat{F}=F-B
$$

Then $\hat{W}, \hat{F}$ satisfy the system (2.1)-(2.6) with $a \equiv b \equiv c \equiv 0$ and a new $\mu$ which still satisfies (2.8), with a different $\epsilon_{0}$. It will be convenient to write this system in detail, but for the sake of simplicity we drop the "^". Thus, the reduced system is 
the following:

$$
\begin{aligned}
& z \Delta W-\Delta^{2} W=\mu(x, y, z) \quad \text { in } \quad D, \\
& \frac{\partial W}{\partial x}=\frac{\partial W}{\partial y}=0 \quad \text { for } y=1,-\infty<x<\infty, \\
& W=\frac{\partial W}{\partial y}=0 \quad \text { for } \quad y=0, x>0, \\
& z F+\beta_{0} \frac{\partial F}{\partial x}-\frac{\partial W}{\partial x}=0 \quad \text { for } \quad y=0, x<0, \\
& 3 \frac{\partial^{3} W}{\partial x^{2} \partial y}+\frac{\partial^{3} W}{\partial y^{3}}+\sigma \frac{\partial^{3} F}{\partial x^{3}}-z \frac{\partial W}{\partial y}=0 \quad \text { for } y=0, x<0, \\
& \frac{\partial^{2} W}{\partial x^{2}}-\frac{\partial^{2} W}{\partial y^{2}}=0 \quad \text { for } y=0, x<0 .
\end{aligned}
$$

In order to gain some understanding of the underlying structure of the boundary conditions of the linear system (2.15)-(2.20), we proceed formally to derive an energy equality. For simplicity we assume that $z>0$; then $W$ may assumed to be real valued. Multiplying (2.15) by $W$ and integrating over $D$, we get, after integration by parts,

$$
\begin{aligned}
\int_{D} \mu W= & \int_{D} W\left(z \Delta W-\Delta^{2} W\right) \\
= & \int_{-\infty}^{0}\left[W \frac{\partial}{\partial y} \Delta W-\frac{\partial W}{\partial y} \Delta W-z W \frac{\partial W}{\partial y}\right]_{y=0} d x \\
& -z \int_{D}|\nabla W|^{2}-\int_{D}(\Delta W)^{2}
\end{aligned}
$$

we have assumed here that $W$ is "appropriately" decaying as $|x| \rightarrow \infty$.

Next,

$$
\int_{D} \frac{\partial^{2} W}{\partial x^{2}} \frac{\partial^{2} W}{\partial y^{2}}=-\int_{D} \frac{\partial W}{\partial x} \frac{\partial^{3} W}{\partial x \partial y^{2}}=\left.\int_{-\infty}^{0} \frac{\partial W}{\partial x} \frac{\partial^{2} W}{\partial x \partial y}\right|_{y=0} d x+\int_{D}\left(\frac{\partial^{2} W}{\partial x \partial y}\right)^{2}
$$

so that

$$
\int_{D}(\Delta W)^{2}=\int_{D}\left[\left(\frac{\partial^{2} W}{\partial x^{2}}\right)+\left(\frac{\partial^{2} W}{\partial y^{2}}\right)^{2}+2\left(\frac{\partial^{2} W}{\partial x \partial y}\right)^{2}\right]+\left.2 \int_{-\infty}^{0} \frac{\partial W}{\partial x} \frac{\partial^{2} W}{\partial x \partial y}\right|_{y=0} d x
$$

By integration by parts

$$
\left.2 \int_{-\infty}^{0} \frac{\partial W}{\partial x} \frac{\partial^{2} W}{\partial x \partial y}\right|_{y=0} d x=-\left.\int_{-\infty}^{0} \frac{\partial^{2} W}{\partial x^{2}} \frac{\partial W}{\partial y}\right|_{y=0} d x
$$

and by $(2.20)$

$$
\left.\int_{-\infty}^{0} \frac{\partial W}{\partial y} \Delta W\right|_{y=0} d x=\left.2 \int_{-\infty}^{0} \frac{\partial W}{\partial y} \frac{\partial^{2} W}{\partial x^{2}}\right|_{y=0}
$$


Substituting (2.22) into (2.21) and using (2.23), (2.24), we find that

$$
\begin{aligned}
& \int_{D} \mu W=\int_{-\infty}^{0} W\left[\frac{\partial}{\partial y} \Delta W-z \frac{\partial W}{\partial y}\right]_{y=0} d x-\int_{D} z|\nabla W|^{2} \\
& -\int_{D}\left[\left(\frac{\partial^{2} W}{\partial x^{2}}\right)^{2}+\left(\frac{\partial^{2} W}{\partial y^{2}}\right)^{2}+2\left(\frac{\partial^{2} W}{\partial x \partial y}\right)^{2}\right] .
\end{aligned}
$$

Next, by integration by parts,

$$
\begin{aligned}
& \int_{D} \frac{\partial^{2} W}{\partial x^{2}} \frac{\partial^{2} W}{\partial y^{2}}=\int_{D}\left(\frac{\partial^{2} W}{\partial x \partial y}\right)^{2}+\left.\int_{-\infty}^{0} \frac{\partial W}{\partial x} \frac{\partial^{2} W}{\partial x \partial y}\right|_{y=0} d x \\
& =\int_{D}\left(\frac{\partial^{2} W}{\partial x \partial y}\right)^{2}-\left.\int_{-\infty}^{0} W \frac{\partial^{3} W}{\partial x^{2} \partial y}\right|_{y=0} d x .
\end{aligned}
$$

This enables us to rewrite $(2.25)$ in the form

$$
\begin{aligned}
& \int_{D} \mu W=\left.\int_{-\infty}^{0} W\left[\frac{\partial}{\partial y} \Delta W-z \frac{\partial W}{\partial y}\right]\right|_{y=0} d x-z \int_{D}|\nabla W|^{2} \\
& -\int_{D}\left[\left(\frac{\partial^{2} W}{\partial x^{2}}\right)^{2}+\left(\frac{\partial^{2} W}{\partial y^{2}}\right)^{2}+4\left(\frac{\partial^{2} W}{\partial x \partial y}\right)^{2}-2 \frac{\partial^{2} W}{\partial x^{2}} \frac{\partial^{2} W}{\partial y^{2}}\right] \\
& +\left.2 \int_{-\infty}^{0} W \frac{\partial^{3} W}{\partial x^{2} \partial y}\right|_{y=0} d x \\
& =\left.\int_{-\infty}^{0} W\left[3 \frac{\partial^{3} W}{\partial x^{2} \partial y}+\frac{\partial^{3} W}{\partial y^{3}}-z \frac{\partial W}{\partial y}\right]\right|_{y=0} d x-z \int_{D}|\nabla W|^{2} \\
& -\int_{D}\left[\left(\frac{\partial^{2} W}{\partial x^{2}}-\frac{\partial^{2} W}{\partial y^{2}}\right)^{2}+4\left(\frac{\partial^{2} W}{\partial x \partial y}\right)^{2}\right] .
\end{aligned}
$$

Using the boundary condition (2.19) we then arrive at the equation

$$
\begin{gathered}
\int_{D}\left[\left(\frac{\partial^{2} W}{\partial x^{2}}-\frac{\partial^{2} W}{\partial y^{2}}\right)^{2}+4\left(\frac{\partial^{2} W}{\partial x \partial y}\right)^{2}\right]+z \int_{D}|\nabla W|^{2} \\
+\left.\sigma \int_{-\infty}^{0} W \frac{\partial^{3} F}{\partial x^{3}}\right|_{y=0} d x=-\int_{D} \mu W .
\end{gathered}
$$


Finally, by integration by parts and (2.18),

$$
\begin{aligned}
& \left.\sigma \int_{-\infty}^{0} W \frac{\partial^{3} F}{\partial x^{3}}\right|_{y=0} d x=-\left.\sigma \int_{-\infty}^{0} \frac{\partial W}{\partial x} \frac{\partial^{2} F}{\partial x^{2}}\right|_{y=0} d x \\
& =\left.\sigma \int_{-\infty}^{0} z\left(\frac{\partial F}{\partial x}\right)^{2}\right|_{y=0} d x+\frac{1}{2} \sigma \beta_{0}\left(\frac{\partial F(0, z)}{\partial x}\right)^{2},
\end{aligned}
$$

where we have also used the fact that, since $W_{x}(x, 0)=0, z F+\beta_{0} \partial_{x} F=0$ at $x=0, y=0$.

Substituting (2.28) into (2.27) we get:

\section{Energy Equality:}

$$
\begin{aligned}
& \int_{D}\left[\left(\frac{\partial^{2} W}{\partial x^{2}}-\frac{\partial^{2} W}{\partial y^{2}}\right)^{2}+4\left(\frac{\partial^{2} W}{\partial x \partial y}\right)^{2}\right]+z \int_{D}|\nabla W|^{2} \\
& +\sigma z \int_{-\infty}^{0}\left(\frac{\partial F(x, z)}{\partial x}\right)^{2} d x+\frac{1}{2} \sigma \beta_{0}\left(\frac{\partial F(0, z)}{\partial x}\right)^{2}=-\int_{D} \mu W .
\end{aligned}
$$

Recalling that $\beta_{0}>0$, we see that each term on the left-hand side in nonnegative. This equality then provides a formal proof of uniqueness in case $z>0$. By Korn's inequality [3, pp. 110-113] the first integral on the left-hand side of (2.29) controls all the second derivatives of $W$ in $L^{2}(D)$. This suggests a proof of existence in a suitable function space. In the following section we shall, in fact, prove existence for $z>0$, and uniqueness for any complex $z$ with $\operatorname{Re} z \geq 0$,

\section{Existence $(z>0)$ And Uniqueness $(\operatorname{Re} z \geq 0)$}

The Lax-Milgram theorem does not seem to provide a convenient framework for proving existence for the system (2.15)-(2.20). We shall instead use a duality argument.

We suppose for now that $z$ is a positive real number.

Denote by $\mathcal{A}$ the class of all smooth pairs $(W, F)$ compactly supported in $\bar{D}$ away from the origin and satisfying the boundary conditions (2.16)-(2.20). To each pair $(W, F)$ in $\mathcal{A}$ we associate a function $\mu$ by $(2.15)$.

Lemma 3.1. The set of functions $\mu$ is dense in $H^{-4}(D)$.

Proof. Suppose the assertion is not true. Then there exists a $\varphi$ in $H^{4}(D)$ such that

$$
\varphi \not \equiv 0 \quad \text { and } \quad \int_{D} \varphi \mu=0 \quad \forall(W, F) \in \mathcal{A},
$$

where the integral is understood by the duality between $H^{4}$ and $H^{-4}$. Since $\mu$ is smooth, we can rewrite the last equation in the form

$$
\int_{D}\left(z \Delta W-\Delta^{2} W\right) \varphi=0 .
$$


If we integrate by parts we obtain boundary integrals, on $y=1$,

$$
\left.\int_{-\infty}^{\infty} \frac{\partial}{\partial y}(\Delta W) \cdot \varphi\right|_{y=1} d x+\left.\int_{-\infty}^{\infty}(\Delta W) \frac{\partial \varphi}{\partial y}\right|_{y=1} d x
$$

and other integrals either over $D$ or over $y=0$. The only restrictions on $W$ at $y=1$ are

$$
\frac{\partial W}{\partial x}=0 \quad \text { and } \quad \frac{\partial W}{\partial y}=0 .
$$

We can therefore choose $W$ such that

$$
W=\frac{c(x)}{2}(y-1)^{2}+\frac{d(x)}{6}(y-1)^{3} \quad \text { near } \quad y=1,
$$

where $c(x), d(x)$ are arbitrary, and truncate $W$ appropriately, away from $y=1$.

Since $\Delta W=c(x), \frac{\partial}{\partial y} \Delta W=d(x)$ at $y=1$ and $c(x), d(x)$ are arbitrary, we deduce that

$$
\varphi=\frac{\partial \varphi}{\partial y}=0 \quad \text { at } y=1, x \in \mathbb{R}
$$

Similarly,

$$
\varphi=\frac{\partial \varphi}{\partial y}=0 \quad \text { at } \quad y=0, x>0 .
$$

From the arbitrariness of $W$ in $D$ we also deduce that

$$
z \Delta \varphi-\Delta^{2} \varphi=0 \text { in } D .
$$

Next we proceed to identify the boundary conditions for $\varphi$ on $y=0, x<0$. Integrating by parts in (3.2) and using (3.3)-(3.5), we get

$$
-\int_{-\infty}^{0}\left[z \frac{\partial W}{\partial y}-\frac{\partial}{\partial y}(\Delta W)\right] \varphi d x+\int_{-\infty}^{0}\left[z W \frac{\partial \varphi}{\partial y}-\Delta W \cdot \frac{\partial \varphi}{\partial y}\right] d x
$$

$$
+\int_{-\infty}^{0} \frac{\partial W}{\partial y} \Delta \varphi d x-\int_{-\infty}^{0} W \frac{\partial}{\partial y}(\Delta \varphi) d x=0
$$

where all the integrands are evaluated at $y=0$. We shall take $W$ such that

$$
W(x, y)=a(x)+b(x) y+\frac{c(x)}{2} y^{2}+\frac{d(x)}{6} y^{3} \quad \text { for } y \text { near } 0, x<0,
$$

and $F, a, b, c, d$ are compactly supported away from the origin. In order for $(W, F)$ to belong to $\mathcal{A}$ we need to impose the following boundary conditions at $y=0$, $x<0$ :

$$
\begin{gathered}
z F(x)+\beta_{0} \frac{\partial F}{\partial x}-\frac{\partial a}{\partial x}=0, \\
3 \frac{\partial^{2} b}{\partial x^{2}}+d(x)+\sigma \frac{\partial^{3} F}{\partial x^{3}}-z b(x)=0, \\
\frac{\partial^{2} a}{\partial x^{2}}-c(x)=0 .
\end{gathered}
$$


These equations are satisfied if we choose $F(x)$ and $b(x)$ arbitrary functions, and $a, c, d$ such that

$$
\begin{gathered}
\frac{\partial a}{\partial x}=z F(x)+\beta_{0} \frac{\partial F}{\partial x}, \quad a(0)=0 \\
c(x)=\frac{\partial^{2} a}{\partial x^{2}}=z \frac{\partial F}{\partial x}+\beta_{0} \frac{\partial^{2} F}{\partial x^{2}} \\
d(x)=z b(x)-\sigma \frac{\partial^{3} F}{\partial x^{3}}-3 \frac{\partial^{2} b}{\partial x^{2}}
\end{gathered}
$$

If we choose $F$ and $b$ compactly supported away from the origin and $\int_{-\infty}^{0} F(x) d x$ $=0$, then the the functions $a, c$ and $d$ are also compactly supported away from the origin.

Substituting (3.7) into (3.6), we obtain

$$
\begin{aligned}
& \int_{-\infty}^{0}\left[z b(x)-\left(\frac{\partial^{2} b}{\partial x^{2}}+d(x)\right)\right] \varphi(x, 0) d x \\
& +\int_{-\infty}^{0}\left[z a(x)-\left(\frac{\partial^{2} a}{\partial x^{2}}+c(x)\right)\right] \frac{\partial \varphi}{\partial y}(x, 0) d x \\
& +\int_{-\infty}^{0} b(x) \Delta \varphi(x, 0) d x-\int_{-\infty}^{0} a(x) \frac{\partial}{\partial y} \Delta \varphi(x, 0) d x=0
\end{aligned}
$$

or, by (3.9), (3.10),

$$
\begin{aligned}
& -\int_{-\infty}^{0}\left[2 \frac{\partial^{2} b}{\partial x^{2}}+\sigma \frac{\partial^{3} F}{\partial x^{3}}\right] \varphi(x, 0) d x+\int_{-\infty}^{0} b(x) \Delta \varphi(x, 0) d x \\
& +\int_{-\infty}^{b}\left[z a(x)-2 \frac{\partial^{2} a}{\partial x^{2}}\right] \frac{\partial \varphi(x, 0)}{\partial y} d x-\int_{-\infty}^{0} a(x) \frac{\partial}{\partial y} \Delta \varphi(x, 0) d x=0 .
\end{aligned}
$$

Choose first $F=0$; then $a=c=0$ (by (3.11), (3.12)), so that (3.14) reduces, after integration by parts, to

$$
-2 \int_{-\infty}^{0} b(x) \frac{\partial^{2} \varphi(x, 0)}{\partial x^{2}} d x+\int_{-\infty}^{0} b(x) \Delta \varphi(x, 0) d x=0
$$

Since $b(x)$ is arbitrary, we find that

$$
\frac{\partial^{2} \varphi}{\partial y^{2}}-\frac{\partial^{2} \varphi}{\partial x^{2}}=0 \quad \text { for } y=0, x<0
$$

Next we choose $b(x)=0$ in (3.14), and integrate by parts to obtain

$$
\sigma \int_{-\infty}^{0} F(x) \frac{\partial^{3} \varphi}{\partial x^{3}}(x, 0) d x+\int_{-\infty}^{0} a(x)\left[z \frac{\partial \varphi}{\partial y}-\frac{\partial^{3} \varphi}{\partial x^{3}}-3 \frac{\partial^{3} \varphi}{\partial x^{2} \partial y}\right](x, 0) d x=0 .
$$


We define a function $H(x)$ by

$$
z H-\beta_{0} \frac{\partial H}{\partial x}=\frac{\partial \varphi}{\partial x} \quad \text { for } \quad x<0, \quad H(0)=0 .
$$

Then

$$
\begin{aligned}
\int_{-\infty}^{0} F(x) & \frac{\partial^{3} \varphi(x, 0)}{\partial x^{3}} d x=\int_{-\infty}^{0} F(x)\left(z \frac{\partial^{2} H}{\partial x^{2}}-\beta_{0} \frac{\partial^{3} H}{\partial x^{3}}\right) d x \\
= & \int_{-\infty}^{0}\left(z F(x)+\beta_{0} \frac{\partial F}{\partial x}\right) \frac{\partial^{2} H}{\partial x^{2}} d x \quad \text { (by integration by parts) } \\
= & -\int_{-\infty}^{0} a(x) \frac{\partial^{3} H}{\partial x^{3}} d x \quad \text { (by (3.11) and integration by parts). }
\end{aligned}
$$

Substituting this into (3.16), we conclude that

$$
-\sigma \int_{-\infty}^{0} a(x) \frac{\partial^{3} H}{\partial x^{3}} d x+\int_{-\infty}^{0} a(x)\left[z \frac{\partial \varphi}{\partial y}-\frac{\partial^{3} \varphi}{\partial y^{3}}-3 \frac{\partial^{3} \varphi}{\partial x^{2} \partial y}\right](x, 0) d x=0 .
$$

Since $a$ is determined by (3.11) whereas $F$ is arbitrary subject to $\int_{-\infty}^{0} F=0$, for any $-\infty<x_{0}<0$ and small $\varepsilon>0$ we can choose $F$ such that $a(x)$ is uniformly bounded if $\left|x-x_{0}\right|>\varepsilon$ and $a(x) \approx \delta\left(x-x_{0}\right)$ if $\left|x-x_{0}\right|<\varepsilon$, where $\delta$ is the Dirac function. It then follows, from the last equation, that

$$
z \frac{\partial \varphi}{\partial y}-\frac{\partial^{3} \varphi}{\partial y^{3}}-3 \frac{\partial^{3} \varphi}{\partial x^{2} \partial y}-\sigma \frac{\partial^{3} H}{\partial x^{3}}=0 \quad \text { for } \quad y=0, x<0 .
$$

Having proved that $\varphi$ and $H$ satisfy (3.5) and the boundary conditions (3.3), (3.4) and (3.15), (3.17), (3.18), we can proceed to derive the energy equality, as in $\S 2$. The fact that $\varphi \in H^{4}(D)$ provides the "appropriate decay" of $\varphi$ at the origin and at $x= \pm \infty$ which is needed to justify the various integrations by parts. The only difference is that $\beta_{0}$ is now replaced by $-\beta_{0}$, so that the energy equality becomes

$$
\begin{aligned}
& \int_{D}\left[\left(\frac{\partial^{2} \varphi}{\partial x^{2}}-\frac{\partial^{2} \varphi}{\partial y^{2}}\right)^{2}+4\left(\frac{\partial^{2} \varphi}{\partial x \partial y}\right)^{2}\right]+z \int_{D}|\nabla \varphi|^{2} \\
& +\left.\sigma z \int_{-\infty}^{0}\left(\frac{\partial H}{\partial x}\right)^{2}\right|_{y=0} d x-\frac{1}{2} \sigma \beta_{0}\left(\frac{\partial H(0)}{\partial x}\right)^{2}=0 .
\end{aligned}
$$

The asymptotic behavior of $\varphi$ as $r \rightarrow 0$ depends on the highest derivatives of $\varphi$ in the boundary conditions. Thus, in view of (3.17), the effect of $\sigma\left(\partial^{3} H / \partial x^{3}\right)$ in (3.18) is the same as that of $-\frac{\sigma}{\beta_{0}}\left(\partial^{3} \varphi / \partial x^{3}\right)$. But then the principal part in this boundary condition coincides with that of the corresponding stationary problem studied in [6]. We can therefore apply (as in [6]) the results of $[5, \S 6]$ to deduce an asymptotic behavior of $\varphi$ near the origin. Since $\varphi \in H^{4}(D)$, the first term in the 
asymptotic expansion is const. $r^{5 / 2}$. It follows that

$$
|\varphi| \leq C r^{5 / 2}, \quad \text { so that (by (3.17)) } \quad|H| \leq C r^{3 / 2} \text { and } \frac{\partial H(0)}{\partial x}=0 .
$$

Therefore we deduce from (3.19) that $\varphi \equiv 0$, which is a contradiction to (3.1).

We need an improvement of Lemma 3.1:

Lemma 3.2. The set of functions $\mu$ is dense in $H^{-2}(D)$.

Proof. We proceed as before to derive a contradiction to (3.1) with $\varphi \in H^{2}(D), \varphi \not \equiv$ 0 . First we derive the elliptic equation (3.5) and the boundary conditions (3.3), (3.4) in a weak sense, as before. By elliptic theory [9] it then follows that $\varphi$ is a smooth solution in $D$, satisfying the boundary conditions at $y=1$ in the classical sense. The same is true of the boundary conditions at $\{y=0, x>0\}$. As for $\{y=0, x<0\}$, using (3.17) we can formally write the boundary condition (3.18) in the form

$$
\begin{aligned}
& \frac{\partial^{3} \varphi}{\partial y^{3}}+3 \frac{\partial^{3} \varphi}{\partial x^{2} \partial y}-\frac{\sigma}{\beta_{0}} \frac{\partial^{3} \varphi}{\partial x^{3}}=A, \quad \text { where } \\
& A=z \frac{\partial \varphi}{\partial y}-\frac{\sigma z}{\beta_{0}^{2}}\left[-\frac{\partial^{2} \varphi}{\partial x^{2}}-\frac{z}{\beta_{0}} \frac{\partial \varphi}{\partial x}+\frac{z}{\beta_{0}^{2}} \int_{x}^{0} e^{z(x-\xi) / \beta_{0}} \varphi(\xi) d \xi\right] .
\end{aligned}
$$

Equation (3.5) with the boundary conditions (3.15), (3.20) (for a given $A$ ) form a coercive elliptic boundary value problem at $y=0, x<0$. Viewing the term $A$ as an inhomogeneous boundary term and using elliptic regularity for solutions satisfying the boundary conditions is a weak sense [9], we can bootstrap the regularity of $\varphi$, away from the origin, to $H^{4}$; thus, for any ball $B_{\epsilon}$ of radius $\epsilon$ and center at the origin, $\varphi \in H^{4}\left(D \backslash B_{\epsilon}\right)$.

Near the origin we use the fact that $\varphi \in H^{2}(D)$ to deduce, by $[5, \S 5]$, the asymptotic behavior

$$
\varphi \sim A_{0} r^{3 / 2}
$$

Hence by $(3.17), H(x) \sim A_{1}(-x)^{3 / 2}$ and $H^{\prime}(0)=0$.

We can now proceed, precisely as in the proof of Lemma 3.1, to derive the energy equality (3.19) and to deduce that $\varphi \equiv 0$, which is a contradiction.

Consider the mapping:

$$
\mathcal{A} \ni(W, F) \rightarrow z \Delta W-\Delta^{2} W=\mu \in H^{-2}(D),
$$

and set $T W=\mu$. Then, for any $\epsilon>0$,

$$
\left|\int_{D} \mu W\right| \leq\|\mu\|_{H^{-2}(D)}\|W\|_{H^{2}(D)} \leq \epsilon\|W\|_{H^{2}(D)}+C_{\epsilon}\|\mu\|_{H^{-2}(D)} .
$$

We need to use Korn's inequality [3, pp. 110-113], which is valid in $\mathbb{R}_{+}^{n}=$ $\left\{x=\left(x_{1}, \ldots, x_{n}\right) \in \mathbb{R}^{n}, x_{n}>0\right\}$ for all $v=\left(v_{1}, \ldots, v_{n}\right)$ with $v_{i} \in H^{1}\left(\mathbb{R}_{+}^{n}\right)$ :

$$
\int_{\mathbb{R}_{+}^{n}} \sum_{i, j=1}^{n}\left(\frac{\partial v_{i}}{\partial x_{j}}\right)^{2} \leq C \int_{\mathbb{R}_{+}^{n}} \sum_{i, j=1}^{n}\left(\frac{\partial v_{i}}{\partial x_{j}}+\frac{\partial v_{j}}{\partial x_{i}}\right)^{2}+c \int_{\mathbb{R}_{+}^{n}} \sum_{i=1}^{n} v_{i}^{2} .
$$


Applying this to $\widetilde{v}_{i}(x)=v_{i}(R x)$ we get the same inequality for $v(x)$ with $c$ replaced by $c / R^{2}$, and letting $R \rightarrow \infty$ we conclude that Korn's inequality holds with $c=0$. We can apply this inequality for $n=2$ and $v_{1}=-\partial W / \partial y, v_{2}=\partial W / \partial x$, after extending $v_{1}$ and $v_{2}$ by zero to $y>1$ (recall that $v_{1}=v_{2}=0$ on $y=1-0$, by $(2.16))$. We then get

$$
\int_{D}\left[\left(\frac{\partial^{2} W}{\partial x^{2}}-\frac{\partial^{2} W}{\partial y^{2}}\right)^{2}+4\left(\frac{\partial^{2} W}{\partial x \partial y}\right)^{2}\right] \geq \frac{1}{C} \int_{D}\left|\nabla^{2} W\right|^{2} .
$$

Observe that since $W_{x}(x, 1) \equiv 0$ and $W(x, y)=0$ if $|x|$ is large, we have $W(x, 1) \equiv 0$. Hence

$$
\int_{D} W^{2} \leq C_{1} \int_{D}|\nabla W|^{2} .
$$

Next, by integration by parts we deduce the energy equality (2.29). Since $z>0$, from the last two estimates and from (2.29) we conclude that

$$
\|W\|_{H^{2}(D)} \leq C\left|\int_{D} \mu W\right| .
$$

Combining this with (3.21), it follows that

$$
\|W\|_{H^{2}(D)} \leq C\|\mu\|_{H^{-2}(D)},
$$

i.e.,

$$
\|W\|_{H^{2}(D)} \leq C\|T W\|_{H^{-2}(D)} .
$$

Now, by Lemma 3.2, the range of $T$ is dense in $H^{-2}(D)$. Hence for any $\mu \in$ $H^{2}(D)$ there is a sequence $\left(W_{m}, F_{m}\right)$ such that $\mu_{m} \equiv T W_{m}$ is convergent to $\mu$ in $H^{-2}(D)$. From (3.22) it follows that

$$
\left\|W_{m}-W\right\|_{H^{2}(D)} \rightarrow 0
$$

for some function $W$ in $H^{2}(D)$, and from (2.18) and a trace theorem we then easily deduce that also

$$
\left\|F_{m}-F\right\|_{H^{3 / 2}} \rightarrow 0 \quad \text { for some } F \text { in } H^{3 / 2},
$$

where $H^{3 / 2}$ is taken over the interval $\{-\infty<x<0\}$. It follows that $(W, F)$ is a "weak solution" of (2.15)-(2.20) in the sense that the boundary conditions are satisfied in the distribution sense (or, more precisely, in the appropriate $H^{-p}$ space).

If, in particular, $\mu \in L^{2}(D)$, then, by elliptic regularity [9] (as in the proof of Lemmas 3.1,3.2), $W$ belongs to $H^{4}\left(D \backslash B_{\epsilon}\right)$ for any ball $B_{\epsilon}$ of radius $\epsilon$ and center at the origin. If further $\mu \in H^{m}(D)$ (or $\mu \in C^{m+\alpha}(\bar{D})$ ), then $W \in H^{m+4}(D)$ (or $W \in C^{m+4+\alpha}\left(D \backslash B_{\epsilon}\right)$, by [1]).

We summarize:

Theorem 3.3. Suppose $z>0$. Then for any $\mu \in L^{2}(D)$ there exists a solution $(W, F)$ of (2.15)-(2.20) with $W \in H^{2}(D)$ and $W \in H^{4}\left(D \backslash B_{\epsilon}\right)$ for any $\epsilon>0$. The solution satisfies the boundary conditions (2.16)-(2.20) in the trace class sense. If, further, $\mu \in H^{m}(D)$ (or $\left.\mu \in C^{m+\alpha}(\bar{D}), 0<\alpha<1\right)$ then $W$ belongs to $H^{m+4}\left(D \backslash B_{\epsilon}\right)$ (or $W \in C^{m+4+\alpha}\left(\bar{D} \backslash B_{\epsilon}\right)$ ) for any $\epsilon>0$.

We next state a uniqueness result. 
Theorem 3.4. If $\operatorname{Re} z \geq 0$ then there exists at most one solution $(W, F)$ of (2.15)(2.20) with $W$ in $H^{2}(D) \cap H^{4}\left(D \backslash B_{\epsilon}\right)$ for any $\epsilon>0$, satisfying the boundary conditions (2.16)-(2.20) in the trace class sense.

Proof. Suppose $\left(W_{1}, F_{1}\right)$ and $\left(W_{2}, F_{2}\right)$ are two solutions, and set $W=W_{1}-W_{2}$, $F=F_{1}-F_{2}$. Then

$$
\int_{D} \bar{W}\left(z \Delta W-\Delta^{2} W\right)=0 .
$$

Integrating by parts as in the case $z>0$ and taking the real part, we end up with the relation

$$
\begin{gathered}
\int_{D}\left[\left|\frac{\partial^{2} W}{\partial x^{2}}-\frac{\partial^{2} W}{\partial y^{2}}\right|^{2}+4\left|\frac{\partial^{2} W}{\partial x \partial y}\right|^{2}\right]+(\operatorname{Re} z) \int_{D}|\nabla W|^{2} \\
+\sigma(\operatorname{Re} z) \int_{-\infty}^{0}\left|\frac{\partial F}{\partial x}\right|^{2} d x+\frac{1}{2} \sigma \beta_{0}\left|\frac{\partial F(0)}{\partial x}\right|^{2}=0,
\end{gathered}
$$

from which the assertion readily follows.

\section{A PRIORI EStimates FOR ELLIPTIC SYSTEMS IN $\mathbb{R}_{+}^{2}$}

Most of this section deals with the Dirichlet problem

$$
\begin{gathered}
z \Delta W-\Delta^{2} W=\mu(x, y) \quad \text { in } \mathbb{R}_{+}^{2}, \\
W(x, 0)=0, \quad W_{y}(x, 0)=0 \quad \text { if } x \in \mathbb{R},
\end{gathered}
$$

where

$$
\text { Re } z \geq 0, \quad z \neq 0
$$

at the end of this section we shall quote a result from [7] concerning an elliptic system in $\mathbb{R}_{+}^{2}$ with "strange" boundary conditions.

We assume that

$$
W(x, y)=0 \quad \text { if }|x|+|y|>2,
$$

and wish to estimate the derivatives of $W$ by a norm of $\mu$ which degenerates at $y=0$; the norm will depend on the parameter $|z|$.

To define this norm we divide the set $\mathbb{R}_{+}^{2} \cap\{|x|<2,|y|<2\}$ into a sequence of squares

$$
\begin{aligned}
& \{j \leq x \leq j+1,1 \leq y \leq 2\},\left\{\frac{j}{2} \leq x \leq \frac{j+1}{2}, \frac{1}{2} \leq y \leq 1\right\} \\
& \left\{\frac{j}{2^{l+1}} \leq x \leq \frac{j+1}{2^{l+1}}, \frac{1}{2^{l+1}} \leq y \leq \frac{1}{2^{l}}\right\}
\end{aligned}
$$

and

$$
\left\{\frac{j}{2^{l+1}} \leq x \leq \frac{j+1}{2^{l+1}}, \quad 0 \leq y \leq \frac{1}{2^{l+1}}\right\}
$$

where $j=0, \pm 1, \pm 2, \ldots$ and $l$ is such that

$$
\frac{1}{2^{l}} \approx \frac{1}{|z|^{1 / 2}}, \quad \text { provided }|z|>1 \text {. }
$$


We denote these squares by $Q_{n}$, their centers by $q_{n}$ and their diameters by $d_{n}$. Clearly

$$
d_{n} \approx \operatorname{dist}\left(q_{n},\{y=0\}\right)
$$

We define

$$
\|\mu\|_{X_{2}}=\sup _{n}\left\{d_{n}\left[f_{Q_{n}}|\mu(x, y)|^{2} d x d y\right]^{1 / 2}\right\}
$$

provided $|z|>1$. If $|z| \leq 1$ then we denote the rectangle $\mathbb{R}_{+}^{2} \cap\{|x|<2,|y|<2\}$ by $Q_{1}$ and set

$$
\|\mu\|_{X_{2}}=\left[\int_{Q_{1}}|\mu(x, y)|^{2} d x d y\right]^{1 / 2} \equiv\|\mu\|_{L^{2}} .
$$

Lemma 4.1. There exists a positive constant $C_{0}$ such that, for any pair $(W, \mu)$ satisfying (4.1)-(4.4), the following estimates hold:

$$
\begin{gathered}
\left\{\int_{-\infty}^{\infty} d x \int_{1 / 4}^{\infty} d y\left[\left|\nabla^{4} W\right|^{2}+|z|^{2}\left|\nabla^{2} W\right|^{2}\right]\right\}^{1 / 2} \leq C_{0}\|\mu\|_{X_{2}}, \\
\sup _{n}\left\{d_{n}\left[\left(f_{Q_{n}}\left|\nabla^{4} W\right|^{2}\right)^{1 / 2}+|z|\left(f_{Q_{n}}\left|\nabla^{2} W\right|^{2}\right)^{1 / 2}\right]\right\} \leq C_{0}\|\mu\|_{X_{2}} .
\end{gathered}
$$

Proof. If $|z| \leq C_{1}$ for a fixed constant $C_{1}$, then (4.5), (4.6) follow from standard elliptic estimates [1], [2]. Thus it is enough to consider the case where $|z|$ is large.

To prove (4.5) we introduce a cutoff function $\eta(y)$ such that

and set

$$
\eta(y)=\left\{\begin{array}{lll}
0 & \text { if } \quad & y<\frac{1}{4} \\
1 & \text { if } \quad y>\frac{3}{8}
\end{array}\right.
$$

$$
\mu_{1}=\eta \mu, \quad \mu_{2}=(1-\eta) \mu .
$$

We also introduce the Green function $Z(x, y, \xi)(x \in \mathbb{R}, y>0, \xi>0)$ for (4.1), (4.2); it satisfies, for each $\xi$,

$$
\begin{aligned}
& z \Delta X-\Delta^{2} Z=\delta(x) \delta(y-\xi) \quad \text { in } \mathbb{R}_{+}^{2}, \\
& Z(x, 0, \xi)=0, \quad Z_{y}(x, 0, \xi)=0 \quad \text { for } x \in \mathbb{R} .
\end{aligned}
$$

We can write $W=W_{1}+W_{2}$, where

$$
W_{j}(x, y)=\int_{\mathbb{R}_{+}^{2}} Z(x-\lambda, y, \xi) \mu_{j}(\lambda, \xi) d \lambda d \xi .
$$

Set

$$
\omega=\frac{z}{|z|}, \quad R=|z|^{1 / 2}
$$


According to $[7, \S 6]$

$$
Z(x, y, \xi)=\frac{1}{R^{2}} V(R x, R y, \theta) \quad(\theta=R \xi),
$$

where

$$
\begin{aligned}
& V(\bar{x}, \bar{y}, \theta)=\varphi(\bar{x}, \bar{y}-\theta)+\varphi(\bar{x}, \bar{y}+\theta)-G_{1}(\bar{x}, \bar{y}, \theta)-\frac{B}{\pi}-G_{2}(\bar{x}, \bar{y}, \theta), \\
& \varphi(\bar{x}, \bar{y}) \equiv \varphi(\bar{r})=\frac{1}{2 \pi} \int_{0}^{\bar{r}} \frac{d u}{u} \int_{0}^{u} \xi K_{0}(\sqrt{\omega} \xi) d \xi, \quad \bar{r}=\left(\bar{x}^{2}+\bar{y}^{2}\right)^{1 / 2},
\end{aligned}
$$

$B$ is a constant, $K_{0}$ is the zero-th order modified Bessel function,

$$
\begin{aligned}
& G_{1}(\bar{x}, \bar{y}, \theta)=2 \Gamma \log \theta+\Gamma \Phi(\bar{x}, \bar{y}) \\
& \Gamma=\frac{1}{2 \pi} \int_{0}^{\infty} \xi K_{0}(\sqrt{\omega} \xi) d \xi \quad \text { (constant) } \\
& \Phi(\bar{x}, \bar{y})=-\int_{-\infty}^{\infty} d k \frac{e^{-|k| \theta}}{|k|}\left[\frac{\sqrt{k^{2}+\omega} e^{-|k| \bar{y}}-|k| e^{-\sqrt{k^{2}+\omega} \bar{y}}}{\sqrt{k^{2}+\omega}-|k|} e^{i k \bar{x}}-1\right],
\end{aligned}
$$

and, finally,

$$
G_{2}(\bar{x}, \bar{y}, \theta)=2 \int_{-\infty}^{\infty} K(\bar{x}-\lambda, \bar{y})\left[\varphi(\lambda, \theta)-\Gamma \log \sqrt{\lambda^{2}+\theta^{2}}-\frac{B}{2 \pi}\right] d \lambda,
$$

where $[7,(4.7)]$

$$
\begin{aligned}
\sqrt{2 \pi} K(\bar{x}, \bar{y})= & \int_{0}^{\infty} e^{i k \bar{x}} \frac{\sqrt{k^{2}+\omega} e^{-k \bar{y}}-k e^{-\sqrt{k^{2}+\omega}} \bar{y}}{\sqrt{k^{2}+\omega}-k} d k \\
& +\int_{-\infty}^{0} e^{i k \bar{x}} \frac{\sqrt{k^{2}+\omega} e^{k \bar{y}}+k e^{-\sqrt{k^{2}+\omega}} \bar{y}}{\sqrt{k^{2}+\omega}+k} d k .
\end{aligned}
$$

Notice that $\Phi(\bar{x}, \bar{y})$ depends on the parameter $\theta$. Notice also that the inequality in (4.5) involves only derivatives of $W$, and therefore the constant $B / \pi$ in (4.10) will not give any contributions and may thus be dropped; this constant however is not to be dropped in the formula for $G_{2}$.

We first estimate the derivatives of $W_{1}$. The function

$$
\widetilde{\varphi}(x, y)=\varphi(R x, R y)
$$

satisfies

$$
\omega R^{2} \Delta \widetilde{\varphi}-\Delta^{2} \widetilde{\varphi}=\delta(x) \delta(y) \text { in } \mathbb{R}^{2} .
$$

Using Fourier analysis it is readily seen that the function

$$
\widetilde{W}(x, y)=\int_{\mathbb{R}^{2}} \widetilde{\varphi}(x-\lambda, y-\xi) g(\lambda, \xi) d \lambda d \xi
$$


satisfies

$$
\int_{\mathbb{R}_{+}^{2}}\left[\left|\nabla^{4} \widetilde{W}\right|^{2}+R^{2}\left|\nabla^{2} \widetilde{W}\right|^{2}\right] \leq C\|g\|_{L^{2}\left(\mathbb{R}^{2}\right)}^{2} .
$$

Therefore, if we define $W_{1,0}$ as the contribution to $W_{1}$ coming from the part

$$
\varphi(\bar{x}, \bar{y}-\theta)+\varphi(\bar{x}, \bar{y}+\theta)
$$

of $Z$ in (4.8), then

$$
\int_{\mathbb{R}_{+}^{2}}\left[\left|\nabla^{4} W_{1,0}\right|^{2}+|z|^{2}\left|\nabla^{2} W_{1,0}\right|^{2}\right] \leq C_{0}\left\|\mu_{1}\right\|_{X_{2}}^{2} .
$$

Set $\bar{x}=R x, \bar{y}=R y, \theta=R \xi$. If $y>\frac{1}{2}$ then $\bar{y}>\frac{1}{2} R$, and from the definition of $K(\bar{x}, \bar{y})$ we see that

$$
\left|\nabla_{(\bar{x}, \bar{y})}^{2} K\right| \leq C \int_{-\infty}^{\infty}|k|^{2} e^{-|k| \bar{y}} d k+C e^{-\gamma R} \leq \frac{C}{R^{3}} \quad(\gamma>0) ;
$$

similarly

$$
\left|\nabla_{(\bar{x}, \bar{y})}^{4} K\right| \leq \frac{C}{R^{5}}
$$

Hence

$$
\left|\nabla_{(\bar{x}, \bar{y})}^{2} G_{2}(\bar{x}, \bar{y}, \theta)\right| \leq C \int_{-\infty}^{\infty} \frac{1}{R^{3}}\left|\varphi(\lambda, \theta)-\Gamma \log \sqrt{\lambda^{2}+\theta^{2}}-\frac{B}{2 \pi}\right| d \lambda .
$$

Substituting $\lambda=\left(u^{2}-\theta^{2}\right)^{1 / 2}$, we get

$$
\left|\nabla_{(\bar{x}, \bar{y})}^{2} G_{2}\right| \leq \frac{C}{R^{3}} \int_{\{|u| \geq \theta\}}|\varphi(u)-\Gamma \log | u\left|-\frac{B}{2 \pi}\right| \frac{|u| d u}{\left(u^{2}-\theta^{2}\right)^{1 / 2}} .
$$

From [7] we have

$$
|\varphi(u)-\Gamma \log | u\left|-\frac{B}{2 \pi}\right| \leq\left\{\begin{array}{lll}
C|\log | u|| & \text { if } \quad|u|<\frac{1}{2}, \\
C e^{-\gamma|u|} & \text { if } \quad|u|>\frac{1}{2} \quad(\gamma>0) .
\end{array}\right.
$$

Therefore, as can easily be seen, the integral in (4.12) is $\leq C$ and, consequently,

$$
\left|\nabla_{(\bar{x}, \bar{y})}^{2} G_{2}(\bar{x}, \bar{y}, \theta)\right| \leq \frac{C}{R^{3}} \quad \text { if } \bar{y} \geq \frac{1}{2} R .
$$

In the same way one can derive the estimate

$$
\left|\nabla_{(\bar{x}, \bar{y})}^{4} G_{2}(\bar{x}, \bar{y}, \theta)\right| \leq \frac{C}{R^{5}} \quad \text { if } \bar{y} \geq \frac{1}{2} R .
$$

Setting

$$
Z_{j}(x, y, \xi)=\frac{1}{R^{2}} G_{j}(R x, R y, \theta) \quad \text { for } j=1,2 \quad(\theta=R \xi),
$$

we conclude that

$$
\left|\nabla^{4} Z_{2}(x, y, \xi)\right|+R^{2}\left|\nabla^{2} Z_{2}(x, y, \xi)\right| \leq \frac{C}{R} \quad \text { if } y \geq \frac{1}{2} .
$$


If we denote by $W_{1,2}$ the contribution to $W_{1}$ which comes from $Z_{2}$, then (4.14) implies that

$$
\int_{-\infty}^{\infty} d x \int_{1 / 2}^{\infty} d y\left[\left|\nabla^{4} W_{1,2}\right|^{2}+|z|^{2}\left|\nabla^{2} W_{1,2}\right|^{2}\right] \leq C_{0}\left\|\mu_{1}\right\|_{X_{2}}^{2} .
$$

Next, by Lemma 5.3 of [7]

$$
\left|\nabla_{(x, y)}^{j} Z_{1}(x, y, \xi)\right| \leq \frac{C}{R^{2}} \quad \text { if }|x| \leq C, \quad 0 \leq y \leq C, \quad \frac{1}{4} \leq \xi \leq C,
$$

for $1 \leq j \leq 4$, where $Z_{1}$ is defined by (4.13). Hence

$$
\left|\nabla^{4} Z_{1}(x, y, \xi)\right|+R^{2}\left|\nabla^{2} Z_{1}(x, y, \xi)\right| \leq C \quad \text { if }|x| \leq C, \quad 0 \leq y \leq C, \quad \frac{1}{4} \leq \xi \leq C .
$$

It follows that the contribution $W_{1,1}$ to $W_{1}$ from the term $Z_{1}$ satisfies the same estimate as (4.15). Thus, together with (4.15), we conclude that the same estimate holds for $W_{1}$.

We now turn to $W_{2}$. This term contains the contribution of $\mu(\lambda, \xi)$ for $0 \leq \xi \leq \frac{3}{8}$. We first estimate the derivatives of

$$
G_{01}(\bar{x}, \bar{y}, \theta)=\varphi(\bar{x}, \bar{y}-\theta)+\varphi(\bar{x}, \bar{y}+\theta)-G_{1}(\bar{x}, \bar{y}, \theta),
$$

where

$$
\bar{x}=R x, \quad \bar{y}=R y, \quad \theta=R \xi .
$$

From the proof of [7, Lemma 5.1] and the fact that $y \geq \frac{1}{2}$ we have

$$
\left|\frac{\partial}{\partial \bar{y}} \Phi(\bar{x}, \bar{y})-\frac{2(\theta+\bar{y})}{\bar{x}^{2}+(\theta+\bar{y})^{2}}\right| \leq \frac{C}{R^{2}}+\frac{C}{R} e^{-(\operatorname{Re} \sqrt{\omega}) \bar{y}}
$$

if $R \geq 1$ (or, in fact, if $R \geq c_{0}$ for some positive constant $c_{0}$ ). Integrating in $\bar{y}$ and using the initial condition $\Phi(\bar{x}, 0)=\log \left(1+\bar{x}^{2} / \theta^{2}\right)$, we obtain

$$
\left|\Phi(\bar{x}, \bar{y})-\log \left[\bar{x}^{2}+(\theta+\bar{y})^{2}\right]+2 \log \theta\right| \leq \frac{C \bar{y}}{R^{2}}+\frac{C \bar{y}}{R} e^{-(\operatorname{Re} \sqrt{\omega}) y} .
$$

On the other hand, by $[7, \S 5]$,

$$
\varphi(\bar{r})=\Gamma \log \bar{r}+\frac{B}{2 \pi}+W(\bar{r}), \quad|W(\bar{r})| \leq C e^{-\gamma \bar{r}} \quad(\bar{r}>0) .
$$

Recalling (4.16) and the definition of $G_{1}$, we see that

$$
\begin{aligned}
G_{01}(\bar{x}, \bar{y}, \theta) & =\frac{\Gamma}{2}\left[\log \left(\bar{x}^{2}+(\theta-\bar{y})^{2}\right)-\log \left(\bar{x}^{2}+(\theta+\bar{y})^{2}\right)\right]+O\left(\frac{1}{R}\right) \\
& =\frac{\Gamma}{2} \log \frac{x^{2}+(\xi-y)^{2}}{x^{2}+(\xi+y)^{2}}+O\left(\frac{1}{R}\right) .
\end{aligned}
$$

We can express the log term in the form

$$
\frac{\Gamma}{2} \log \left(1-\frac{4 \xi y}{x^{2}+(\xi+y)^{2}}\right)=O\left(\frac{\xi y}{x^{2}+y^{2}}\right),
$$


since $\xi \leq \frac{3}{8}, y \geq \frac{1}{2}$. Setting

$$
Z_{01}(x, y, \xi)=\frac{1}{R^{2}} G_{01}(\bar{x}, \bar{y}, \theta)
$$

we then have

$$
\left|Z_{01}\right| \leq \frac{C|\xi|}{R^{2}}
$$

Similarly,

$$
\left|\nabla_{(x, y)}^{j} Z_{01}\right| \leq \frac{C|\xi|}{R^{2+j}} \quad(0 \leq j \leq 4)
$$

and, in particular,

$$
\left|\nabla^{4} Z_{01}(x, y, \xi)\right|+R^{2}\left|\nabla^{2} Z_{01}(x, y, \xi)\right| \leq C|\xi| .
$$

Notice that

$$
\text { if } \quad(\lambda, \xi) \in Q_{n}, \quad \text { then }|\xi| \leq 2 d_{n} \text {. }
$$

Also,

$$
\frac{1}{R} \leq C d_{n} \text { for all } n
$$

Hence, by combining (4.19) with (4.14), we obtain

$$
\left|\nabla^{4} Z(x-\lambda, y, \xi)\right|+R^{2}\left|\nabla^{2} Z(x-\lambda, y, \xi)\right| \leq C d_{n}
$$

for $(x-\lambda, \xi)$ in $Q_{n}$ or (by the definitions of the $\left.Q_{n}, d_{n}\right)$ for $(\lambda, \xi)$ in $Q_{n}$. It follows that

$$
\begin{gathered}
\left|\nabla^{4} W_{2}\right|+R^{2}\left|\nabla^{2} W_{2}\right| \leq C \sum_{n} \int_{Q_{n}} d_{n}\left|\mu_{2}(\lambda, \xi)\right| d \lambda d \xi \\
\leq C \sum_{n} d_{n}\left|Q_{n}\right|\left(f_{Q_{n}}\left|\mu_{2}\right|^{2}\right)^{1 / 2} \leq C \sum_{n}\left|Q_{n}\right|\left\|\mu_{2}\right\|_{X_{2}} \leq C\|\mu\|_{X_{2}}
\end{gathered}
$$

if $y \geq \frac{1}{2}$. Integrating in $(x, y)$ we conclude that (4.5) holds also for $W_{2}$, and thus the proof of (4.5) is complete.

To prove (4.6) we take a specific $Q_{j}$ and prove that

$$
d_{j}\left\{\left(\int_{Q_{j}}\left|\nabla^{4} W\right|^{2}\right)^{1 / 2}+|z|\left(\int_{Q_{j}}\left|\nabla^{2} W\right|^{2}\right)^{1 / 2}\right\} \leq C_{0}\|\mu\|_{X_{2}} .
$$

Denote the center of the square $Q_{j}$ by $\left(x_{j}, y_{j}\right)$, so that $d_{j} \approx y_{j}$, and take for simplicity $x_{j}=0$. Let $S_{j}$ be the square $|x|<\bar{C} d_{j}, 0 \leq y \leq 2 \bar{C} d_{j}$, where $\bar{C}$ is very large, and denote by $\mathcal{X}_{S_{j}}$ the characteristic function of $S_{j}$. We break $W$ into $W_{1}+W_{2}$, as in (4.8), where

$$
\mu_{1}=\mu \mathcal{X}_{S_{j}}, \quad \mu_{2}=\mu\left(1-\mathcal{X}_{S_{j}}\right) .
$$

We wish to estimate $W_{1}$ in $Q_{j}$ by means of the estimate (4.5). To do that we introduce scaling:

$$
\widetilde{W}_{1}(\bar{x}, \bar{y})=W_{1}\left(d_{j} \bar{x}, d_{j} \bar{y}\right)
$$


for $\bar{x}^{2}+\bar{y}^{2} \leq 4 \bar{C}^{2}$. Clearly

$$
d_{j}^{2} z \Delta \widetilde{W}_{1}-\Delta^{2} \widetilde{W}_{1}=d_{j}^{4} \mu_{1}\left(d_{j} \bar{x}, d_{j} \bar{y}\right) \equiv \widetilde{\mu}_{1}(\bar{x}, \bar{y}) .
$$

Setting $\widetilde{d}_{n}=d_{n} / d_{j}$, we have

$$
\left\|\widetilde{\mu}_{1}\right\|_{X_{2}}=\sup _{n} \widetilde{d}_{n}\left(\int_{\widetilde{Q}_{n}}\left|\widetilde{\mu}_{1}\right|^{2}\right)^{1 / 2}, \quad \text { where } \quad \widetilde{Q}_{n}=\frac{1}{d_{j}} Q_{n},
$$

or

$$
\left\|\widetilde{\mu}_{1}\right\|_{X_{2}}=\sup _{n}\left\{\frac{d_{n}}{d_{j}} d_{j}^{4}\left(\int_{Q_{n}}\left|\mu_{1}\right|^{2}\right)^{1 / 2}\right\}=d_{j}^{3}\left\|\mu_{1}\right\|_{X_{2}} .
$$

The proof of (4.5) does not require that $W$ be compactly supported, only that the integral representation (by Green's function) holds with $\mu$ compactly supported. Hence we can apply it to $W_{1}$. If $d_{j} \geq 2 /|z|^{1 / 2}$ then $\widetilde{Q}_{j}$ lies in $\{y \geq 1\}$ and so, by the proof of (4.5),

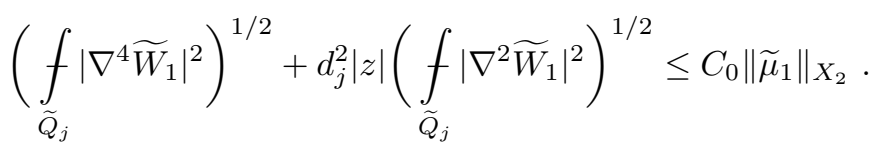

On the other hand, if $d_{j} \leq 2 /|z|^{1 / 2}$ then $d_{j}^{2}|z| \approx 1$, and we can apply standard elliptic estimates to (4.21) to deduce (4.23). Scaling back in (4.23) and using (4.22), we obtain

$$
d_{j}^{4}\left\{\left(f_{Q_{j}}\left|\nabla^{4} W_{1}\right|^{2}\right)^{1 / 2}+|z|\left(f_{Q_{j}}\left|\nabla^{2} W_{1}\right|^{2}\right)^{1 / 2}\right\} \leq C_{0} d_{j}^{3}\left\|\mu_{1}\right\|_{X_{2}},
$$

and (4.20) follows for $W_{1}$.

To prove (4.20) for $W_{2}$ we first consider the contribution to $W_{2}$ coming from

$$
W_{22}(x, y) \equiv \int_{\mathbb{R}_{+}^{2}} Z_{2}(x-\lambda, y, \xi) \mu_{2}(\lambda, \xi) d \lambda d \xi,
$$

where

$$
Z_{2}(x, y, \xi)=\frac{1}{R^{2}} G_{2}(\bar{x}, \bar{y}, \theta)
$$

and $\bar{x}=R x, \bar{y}=R y, \theta=R \xi, R=|z|^{1 / 2} \geq 1$. Note that if $(x, y) \in Q_{j}$ and $(\lambda, \xi) \in \sup \mu_{2}$, then

$$
\left(|x-\lambda|^{2}+\xi^{2}\right)^{1 / 2} \approx\left(\lambda^{2}+\xi^{2}\right)^{1 / 2}>\bar{C} d_{j}, \quad y \leq \eta_{0} d_{j} \quad\left(\eta_{0}=2\right),
$$

and $\eta_{0} / \bar{C} \ll 1$. Hence we can apply Lemma 6.1 of $[7]$ to deduce that

$$
\left|\nabla^{4} Z_{2}(x-\lambda, y, \xi)\right|+R^{2}\left|\nabla^{2} Z_{2}(x-\lambda, y, \xi)\right| \leq \frac{C}{R^{3} \rho^{5}}+\frac{C}{R^{3} \rho^{3}},
$$


where $\rho=\left(\lambda^{2}+\xi^{2}\right)^{1 / 2}$. It follows that

$$
\left|\nabla^{4} W_{22}\right|+R^{2}\left|\nabla^{2} W_{22}\right| \leq \frac{C}{R} \int_{B_{2}^{+} \backslash B_{2 d_{j}}^{+}} \frac{\left|\mu_{2}(\lambda, \xi)\right|}{\rho^{3}}+\frac{C}{R^{3}} \int_{B_{2}^{+} \backslash B_{2 d_{j}}^{+}} \frac{\left|\mu_{2}(\lambda, \xi)\right|}{\rho^{5}} \equiv I_{1}+I_{2}
$$

in $Q_{j}$, where $B_{\gamma}^{+}=\left\{\lambda^{2}+\xi^{2}<\gamma^{2}, \xi>0\right\}$.

Next we evaluate

$$
\begin{aligned}
I_{1} & \leq \frac{C}{R} \sum_{Q_{n} \subset B_{2}^{+} \backslash B_{d_{j}}^{+}}\left(\sup \frac{1}{\rho^{3}}\right) \int_{Q_{n}}\left|\mu_{2}\right| \\
& \leq \frac{C}{R} \sum_{Q_{n} \subset B_{2}^{+} \backslash B_{d_{j}}^{+}}\left(\sup \frac{1}{\rho^{3}}\right) \frac{1}{d_{n}}\left|Q_{n}\right|\left\|\mu_{2}\right\|_{X_{2}} .
\end{aligned}
$$

Since $d_{n} \geq \frac{1}{2}(|\lambda|+1 / R)$ if $(\lambda, \xi) \in Q_{n}$, we obtain

$$
I_{1} \leq \frac{C}{R}\|\mu\|_{X_{2}} \int_{B_{2}^{+} \backslash B_{d_{j}}^{+}} \frac{1}{|\lambda|^{3}+|\xi|^{3}} \frac{d \lambda d \xi}{|\lambda|+1 / R} .
$$

Substituting $(\lambda, \xi)=d_{j}(u, v)$, we get

$$
\begin{aligned}
I_{1} & \leq C\|\mu\|_{X_{2}} \int_{B_{2 / d_{j}}^{+} \backslash B_{1}^{+}} \frac{d_{j}^{2}}{d_{j}^{3}\left(|u|^{3}+|v|^{3}\right)} \frac{d u d v}{R d_{j}|u|+1} \\
& \left.\leq \frac{C}{d_{j}}\|\mu\|_{X_{2}} \int_{B_{2 / d_{j}}^{+} \backslash B_{1}^{+}} \frac{1}{|u|^{3}+|v|^{3}} \frac{d u d v}{|u|+1} \quad \quad \quad \text { (since } R d_{j} \geq 1\right) \\
& \leq \frac{C}{d_{j}}\|\mu\|_{X_{2}} .
\end{aligned}
$$

Similarly we find that

$$
I_{2} \leq \frac{C}{d_{j}}\|\mu\|_{X_{2}} \frac{1}{R^{2} d_{j}^{2}} \leq \frac{C}{d_{j}}\|\mu\|_{X_{2}}
$$

so that

$$
\left|\nabla^{4} W_{2,2}\right|+R^{2}\left|\nabla^{2} W_{2,2}\right| \leq \frac{C}{d_{j}}\|\mu\|_{X_{2}} \quad \text { in } Q_{j} .
$$

Averaging over $Q_{j}$, we obtain the estimate (4.6) for $W_{2,2}$.

It remains to estimate the contribution to $W_{2}$ coming from

$$
W_{2,10}(x, y) \equiv \int_{\mathbb{R}_{+}^{2}} Z_{01}(x-\lambda, y, \xi) \mu_{2}(\lambda, \xi) d \lambda d \xi,
$$

where $Z_{01}$ is defined in (4.18). Using (4.17) we can derive, for $(x, y) \in Q_{j}$ and $(\lambda, \xi) \in \sup \mu$, the estimate

$$
\left|\nabla^{i} Z_{01}(x-\lambda, y, \xi)\right| \leq \frac{C}{R^{2}} \frac{|\xi|+1 / R}{\rho^{i+1}}, \quad \rho=\left(\lambda^{2}+\xi^{2}\right)^{1 / 2}
$$


where the term $1 / R$ (occurring in $|\xi|+1 / R$ ) is introduced in order to bound the derivatives $\nabla^{j}$ of the $O(1 / R)$ terms in (4.17). We conclude that

$$
\begin{aligned}
& \int_{Q_{j}}\left|\nabla^{4} W_{2,10}\right|^{2}+R^{2}\left(\int_{Q_{j}}\left|\nabla^{2} W_{2,10}\right|^{2}\right)^{1 / 2} \\
& \leq C \int_{B_{2}^{+} \backslash B_{2 d_{j}}^{+}}\left(\frac{|\xi|+1 / R}{\rho^{3}}+\frac{|\xi|+1 / R}{\xi^{5} R^{2}}\right)\left|\mu_{2}\right| .
\end{aligned}
$$

Decomposing $B_{2}^{+} \backslash B_{2 d_{j}}^{+}$into squares $Q_{k}$ and proceeding as in the estimates for $I_{1}$ and $I_{2}$ above, we find that the right-hand side is bounded by $C\|\mu\|_{X_{2}} / d_{j}$, so that (4.6) holds also for $W_{2,10}$. This completes the proof of (4.6) for $W_{2}$ and thus also for $W$.

Lemma 4.2. There exists a positive constant $C_{0}$ such that for any pair $(W, \mu)$ satisfying (4.1)-(4.4) the following estimate holds:

$$
\sup _{n} \frac{|z|}{\left|\log d_{n}\right|}\left(f_{Q_{n}}|\nabla W|^{2}\right)^{1 / 2} \leq C_{0}\|\mu\|_{X_{2}} .
$$

Proof. We represent $W$ in $Q_{n}$ by Green's function $Z$ (cf. (4.8)):

$$
W(x, y)=\int_{\mathbb{R}_{+}^{2}} Z(x-\lambda, y, \xi) \mu(\lambda, \xi) d \lambda d \xi
$$

and then, by differentiating and using (4.9),

$$
\nabla W(x, y)=\frac{1}{R} \int_{\mathbb{R}_{+}^{2}}(\nabla V)(R(x-\lambda), R y, R \xi) \cdot \mu(\lambda, \xi) d \lambda d \xi,
$$

where $R=|z|^{1 / 2}$ and $\nabla V=\nabla_{(\bar{x}, \bar{y})} V$. Scaling

$$
x=d_{n} \widetilde{x}, \quad y=d_{n} \widetilde{y}
$$

(we assume for simplicity that the square $Q_{n}$ is centered at the origin) and substituting

$$
\lambda=d_{n} u, \quad \xi=d_{n} v,
$$

we obtain

$$
\nabla W(x, y)=\frac{d_{n}^{2}}{R} \int_{B_{2 / d_{n}}(O) \cap \mathbb{R}_{+}^{2}}(\nabla V)\left(R d_{n}(\widetilde{x}-u), R d_{n} \widetilde{y}, R d_{n} v\right) \mu\left(d_{n} u, d_{n} v\right) d u d v,
$$

where $B_{\rho}(O)$ denotes the disc with radius $\rho$ centered at the origin $O$. Under the above scaling the square $Q_{n}$ becomes another square

$$
\frac{1}{d_{n}} Q_{n} \equiv Q_{1} .
$$

We shall denote by $Q_{2}$ the square $2 Q_{1}$, and break the integral in (4.26) into three parts: one part over $Q_{2}$, and the other two parts in accordance with $v \geq \frac{1}{4}$ and 
$v \leq \frac{1}{4}$. Thus

$$
\begin{aligned}
& \nabla W=\left\{\frac{d_{n}^{2}}{R} \int_{\left[\left(B_{2 / d_{n}}(O) \cap \mathbb{R}_{+}^{2}\right) \cap\left\{v \leq \frac{1}{4}\right\}\right] \backslash Q_{2}}(\nabla V)\left(R d_{n}(\widetilde{x}-u), R d_{n} \widetilde{y}, R d_{n} v\right)\right. \\
& \cdot \mu\left(d_{n} u, d_{n} v\right) d u d v \\
& +\frac{d_{n}^{2}}{R} \int_{Q_{2}}(\nabla V)\left(R d_{n}(\widetilde{x}-u), R d_{n} \widetilde{y}, R d_{n} v\right) \cdot \mu\left(d_{n} u, d_{n} v\right) d u d v \\
& +\frac{d_{n}^{2}}{R} \int_{\left[\left(B_{2 / d_{n}}(O) \cap \mathbb{R}_{+}^{2}\right) \cap\left\{v \geq \frac{1}{4}\right\}\right] \backslash Q_{2}}(\nabla V)\left(R d_{n}(\widetilde{x}-u), R d_{n} \widetilde{y}, R d_{n} v\right) \\
& \left.\cdot \mu\left(d_{n} u, d_{n} v\right) d u d v\right\} \\
& \equiv I_{1}+I_{2}+I_{3} .
\end{aligned}
$$

Notice that $R d_{n} \geq 1$.

We claim that

$$
|(\nabla V)(\bar{x}, \bar{y}, \theta)| \leq \frac{C \theta}{\bar{x}^{2}+\theta^{2}} \quad \text { if } \bar{x}=R d_{n}(\widetilde{x}-u), \bar{y}=R d_{n} \widetilde{y}, \theta=R d_{n} v,
$$

and

$$
(u, v) \in\left[\left(B_{2 / d_{n}}(O) \cap \mathbb{R}_{+}^{2}\right) \cap\left\{v \geq \frac{1}{4}\right\} \cap Q_{2}\right] .
$$

To prove it we recall the structure of $V$ as given in (4.10). $\nabla G_{2}$ is estimated by $O\left(1 / R^{2}\right)$ if $\widetilde{y} \geq 1$ (the proof is the same for (4.14)) or, more generally, if $\widetilde{y} \geq \eta_{0}$ for any small positive number $\eta_{0}$. On the other hand, if $\widetilde{y} \leq \eta_{0}$ then we get the same bound on $\nabla G_{2}$ by using Lemma 6.1 of [7]. The estimates of the remaining terms of $\nabla V$ are obtained by using (6.10), (6.13) of [7] and Lemma 5.1 of [7]. Altogether we obtain, for the first component of $\nabla V$,

$$
\frac{\Gamma}{2} \frac{2 \bar{x}}{\bar{x}^{2}+(\bar{y}-\theta)^{2}}+\frac{\Gamma}{2} \frac{2 \bar{x}}{\bar{x}^{2}+(\bar{y}+\theta)^{2}}-2 \Gamma \frac{\bar{x}}{\bar{x}^{2}+(\bar{y}+\theta)^{2}}+O\left(\frac{1}{R^{2}}\right),
$$

where the first two terms come from $\varphi$ and the third one from $\Phi$, from which (4.28) follows for the first component of $\nabla V$. The second component is estimated in the same way.

Note that in $I_{3} \quad(\widetilde{x}-u)^{2}+v^{2} \approx u^{2}+v^{2}$. Using (4.28) we then obtain

$$
\left|I_{3}\right| \leq C \frac{d_{n}^{2}}{R} \frac{1}{R d_{n}} \int_{\Omega_{n}} \frac{v}{u^{2}+v^{2}}\left|\mu\left(d_{n} u, d_{n} v\right)\right| d u d v,
$$

where $\Omega_{n}$ is the domain of integration in $I_{3}$. Set

$$
\widetilde{\mu}(u, v)=\mu\left(d_{n} u, d_{n} v\right), \quad \widetilde{Q}_{l}=\frac{Q_{l}}{d_{n}} .
$$


Then by the change of variables we find that

$$
\left(\int_{\widetilde{Q}_{l}}|\widetilde{\mu}(u, v)|^{2} d u d v\right)^{1 / 2} \leq \frac{C}{d_{l}}\|\mu\|_{X_{2}}
$$

obviously also

$$
\widetilde{d}_{l} \equiv \operatorname{diam}\left(\widetilde{Q}_{l}\right)=\frac{d_{l}}{d_{n}} .
$$

Breaking up $\Omega_{n}$ into squares $\widetilde{Q}_{l}$ and noting that

$$
1 \leq \widetilde{d}_{l} \leq \frac{1}{d_{n}}
$$

we get

$$
\begin{aligned}
\left|I_{3}\right| & \leq \frac{C d_{n}}{R^{2}} \sum_{1 \leq \tilde{d}_{l} \leq \frac{1}{d_{n}}}\left(\int_{\widetilde{Q}_{l} \cap \Omega_{n}} \frac{v^{2} d u d v}{\left(u^{2}+v^{2}\right)^{2}}\right)^{1 / 2}\left(\int_{\widetilde{Q}_{l} \cap \Omega_{n}}|\widetilde{\mu}(u, v)|^{2} d u d v\right)^{1 / 2} \\
& \leq \frac{C}{R^{2}} \sum_{1 \leq \widetilde{d}_{l} \leq \frac{1}{d_{n}}}\left(\int_{\widetilde{Q}_{l} \cap \Omega_{n}} \frac{v^{2} d u d v}{\left(u^{2}+v^{2}\right)^{2}}\right)^{1 / 2}\|\mu\|_{X_{2}} \\
& \leq \frac{C}{R^{2}}\left|\log d_{n}\right|\|\mu\|_{X_{2}} .
\end{aligned}
$$

We next estimate $I_{2}$. Observe that $I_{2}=(\nabla G)(\widetilde{x}, \widetilde{y})$, where

$$
\omega R^{2} d_{n}^{2} \Delta G-\Delta^{2} G=\widetilde{\mu} \mathcal{X}_{Q_{2}} d_{n}^{2}
$$

and $G=G_{\widetilde{y}}=0$ at $\widetilde{y}=0$. We can proceed as in the proof of (4.20) for $W=W_{1}$ (in fact $G$ is estimated as $\widetilde{W}_{1}$ ), and so we obtain, similarly to (4.23),

$$
R^{2} d_{n}^{2}\left(\int_{Q_{2}}\left|\nabla^{2} G\right|^{2}\right)^{1 / 2} \leq \frac{C d_{n}^{4}}{d_{n}}\|\mu\|_{X_{2}},
$$

where (4.30) has also been used. Since $\nabla G(\widetilde{x}, 0)=0$, the estimate (4.32) yields, by integration,

$$
R^{2} d_{n}^{2}\left(\int_{Q_{2}}|\nabla G|^{2}\right)^{1 / 2} \leq C d_{n}^{2}\|\mu\|_{X_{2}}
$$

so that

$$
\left(f_{Q_{2}}\left|I_{2}\right|^{2}\right)^{1 / 2} \leq \frac{C}{R^{2}}\|\mu\|_{X_{2}} .
$$

We finally estimate $I_{1}$. The bound (4.28) remains valid here and, since $v \leq \frac{1}{4}$, we get

$$
\left|I_{1}\right| \leq C \frac{d_{n}^{2}}{R} \frac{1}{R d_{n}} \int_{\left\{|u| \leq \frac{1}{d_{n}}, 0 \leq v \leq \frac{1}{4}\right\}} \frac{v}{u^{2}+v^{2}}|\widetilde{\mu}(u, v)| d u d v
$$


Roughly,

$$
|\widetilde{\mu}(u, v)| \leq \frac{C}{v}\|\mu\|_{X_{2}},
$$

from which we get the bound

$$
\left|I_{1}\right| \leq \frac{C}{R^{2}} d_{n}\left|\log d_{n}\right|\|\mu\|_{X_{2}} .
$$

One can establish this estimate rigorously by breaking the domain of integration into squares $Q_{l}$ and using (4.30).

Combining (4.34) with (4.33), (4.29) and (4.27), the estimate (4.25) follows.

We shall need later on an estimate for the solution of the elliptic equation

$$
\varepsilon z \Delta W-\Delta^{2} W=\mu(x, y) \text { in } \mathbb{R}^{2},
$$

where

$$
0<\varepsilon \leq 1, \quad \operatorname{Re} z \geq 0, \quad z \neq 0,
$$

subject to the "strange" boundary conditions at $y=0$ :

$$
\begin{array}{r}
z F+\beta_{0} \frac{\partial F}{\partial x}-\frac{\partial W}{\partial x}=a(x), \\
\frac{\partial^{3} W}{\partial x^{2} \partial y}+\frac{\partial^{3} W}{\partial y^{3}}+\sigma \frac{\partial^{3} F}{\partial x^{3}}-\varepsilon z \frac{\partial W}{\partial y}=b(x), \\
\frac{\partial^{2} W}{\partial x^{2}}-\frac{\partial^{2} W}{\partial y^{2}}=c(x) .
\end{array}
$$

We introduce the "norms"

$$
\begin{aligned}
& \|(a, b, c, \mu)\|_{0}=\left\{\int_{-\infty}^{\infty} d x \int_{0}^{\infty} d y|\mu(x, y)|^{2}\right. \\
& \left.+\int_{-\infty}^{\infty} d x\left[\left|D_{x}^{5 / 2} a\right|^{2}+\left|D_{x}^{1 / 2} b\right|^{2}+|\varepsilon z|^{1 / 2}|b|^{2}+\left|D_{x}^{3 / 2} c\right|^{2}+|\varepsilon z|^{3 / 2}|c|^{2}\right]\right\}^{1 / 2}
\end{aligned}
$$

$$
\begin{gathered}
\|||(W, F)||\|_{0}=\left\{\int_{-\infty}^{\infty} d x \int_{0}^{\infty} d y\left[\left|\nabla^{4} W\right|^{2}+|\varepsilon z|^{2}\left|\nabla^{2} W\right|^{2}\right]\right. \\
+\int_{-\infty}^{\infty} d x\left[|\varepsilon z|^{1 / 2}\left|\nabla^{3} W(x, 0)\right|^{2}+|\varepsilon z|^{2}\left|\nabla^{3 / 2} W(x, 0)\right|^{2}+|\varepsilon z|^{3 / 2}\left|\nabla^{2} W(x, 0)\right|^{2}\right. \\
\left.\left.+|\varepsilon z|^{2+1 / 2}\left|\partial_{y} W(x, 0)\right|^{2}\right]+\int_{\infty}^{\infty} d x\left[\left|D_{x}^{7 / 2} F\right|^{2}+|z|\left|D_{x}^{3} F\right|^{2}+|z|^{2}\left|D_{x}^{5 / 2} F\right|^{2}\right]\right\}^{1 / 2} .
\end{gathered}
$$

We shall need a priori estimates under the assumption that

$$
W(x, y)=0 \quad \text { if } x^{2}+y^{2}>R_{0}^{2}
$$

for some constant $R_{0}$. 
Lemma 4.3. There exists a constant $C_{0}$ depending only on $R_{0}$ such that, for any solution $(W, F)$ of (4.35)-(4.39) satisfying (4.42),

$$
\|(W, F) \mid\|_{0} \leq C_{0}\|(a, b, c, \mu)\|_{0} .
$$

This is precisely Lemma 13.3 of [7], except that here we have added to the definition of $\||(W, F)|\|_{0}$ the term

$$
\left[\int_{-\infty}^{\infty} d x\left|D_{x}^{7 / 2} F\right|^{2} d x\right]^{1 / 2} .
$$

The fact that this term may be included, i.e., that this term is bounded by the right-hand side of (4.43), follows from (13.52) of [7] upon multiplying by $|k|$ and using the definition (4.40).

\section{A PRIORI ESTIMATES: LOCALIZATION}

Theorem 3.3 asserts the existence of a solution in $H^{2}(D)$ to the linear system (2.15)-(2.20) in case $z>0$; Theorem 3.4 asserts uniqueness in case $\operatorname{Re} z \geq 0$. The same results hold for the system (2.1)-(2.6).

We need to establish existence for all complex $z$ with Re $z \geq 0, z \neq 0$, and to derive estimates which depend very precisely on the parameter $z$ and on the variable $(x, y)$, especially for $|z|$ large and $(x, y)$ near $(0,0)$.

In this section we shall state these estimates for $(2.1)-(2.6)$; the proof is given in Sections $6-10$ and 14, 15 .

We introduce a continuous positive function $g(R, z)$ defined for $R>0,|z| \geq 1$ such that $g(R, z)=1$ if $R>1$ whereas, for $R \leq 1$,

$$
\frac{1}{g(R, z)}= \begin{cases}R^{\frac{1}{2}-\varepsilon} & \text { if } \quad R>\frac{1}{|z|^{1 / 2}}, \\ \left(\frac{1}{|z|^{1 / 2}}\right)^{-2 \varepsilon} R^{\frac{1}{2}+\varepsilon} & \text { if } \quad \frac{1}{|z|} \leq R \leq \frac{1}{|z|^{1 / 2}}, \\ \left(\frac{1}{|z|^{1 / 2}}\right)^{-2 \varepsilon}\left(\frac{1}{|z|}\right)^{2 \varepsilon}|z| R^{\frac{3}{2}-\varepsilon} & \text { if } \quad R \leq \frac{1}{|z|},\end{cases}
$$

where $\varepsilon$ is any small positive number.

The choice of $g(R, z)$ is made in order to be able to apply Liouville type theorems [7] for blow-up limits which will appear in the proof of the a priori estimates.

We cover the strip D by regions $H_{j}^{1}, H_{j}^{2}$ and $L_{j}^{1}, L_{j}^{2}, L_{j}^{0}$ defined as follows:

$$
\begin{array}{ll}
L_{j}^{0}=\left\{j \leq x \leq j+2, \quad \frac{1}{4} \leq y \leq 1\right\} & (j=0, \pm 1, \pm 2, \ldots), \\
H_{j}^{1}=\left\{-(j+2) \leq x \leq-j, \quad 0 \leq y \leq \frac{3}{4}\right\} & (j=1,2,3, \ldots),
\end{array}
$$

$H_{j}^{2} \quad(j=1,2,3, \ldots)$ has the form shown in Figure 5.1 , and $L_{j}^{1}, L_{j}^{2}$ are the reflections of $H_{j}^{1}, H_{j}^{2}$, respectively, with respect to the $y$-axis. Here $\alpha_{j}, \beta_{j}, \gamma_{j}, \delta_{j}$ are monotone decreasing in $j, \gamma_{1}=1, \alpha_{1}=\beta_{1}=2$ and

$$
j \alpha_{j}, j \beta_{j}, j \gamma_{j}, j \delta_{j}
$$




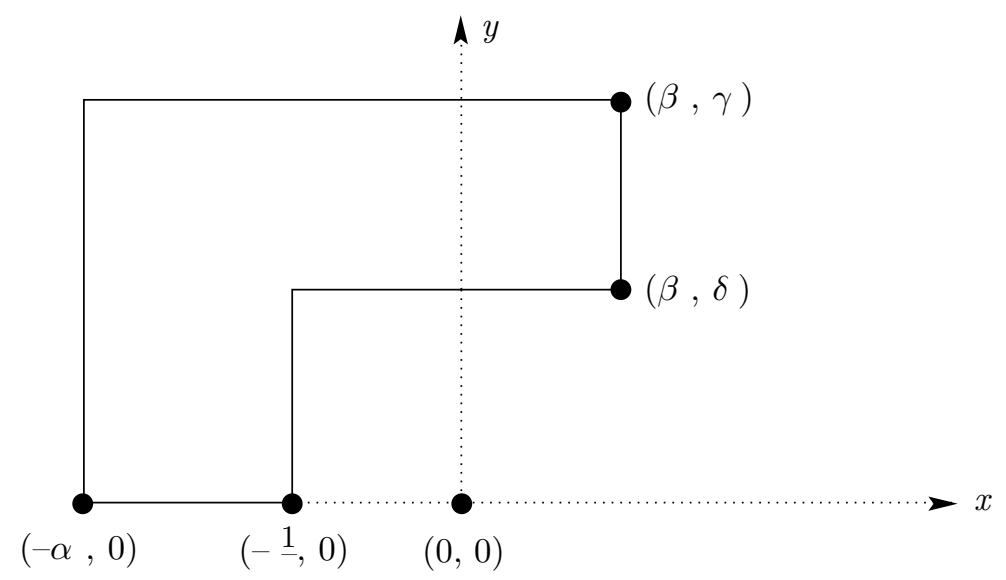

Figure 5.1

are bounded from above and below by positive constants.

The letter " $H$ " in $H_{j}^{1}, H_{j}^{2}$ is to remind us that the boundary conditions (2.4)(2.6) on the boundary of $H_{j}^{1}, H_{j}^{2}$ at $y=0$ are of high order, whereas the letter " $L$ " in $L_{j}^{0}, L_{j}^{1}, L_{j}^{2}$ is to remind us that the boundary conditions are of low order (namely, $W=\partial_{y} W=0$ on $y=0$ and $\partial_{x} W=\partial_{y} W=0$ on $y=1$ ).

It will be convenient to denote the collection of regions $H_{j}^{1}, H_{j}^{2}$ by $S_{k}^{(1)}$ and the collection of regions $L_{j}^{0}, L_{j}^{1}, L_{j}^{2}$ by $S_{k}^{(2)}$. The collection of $S_{k}^{(1)}, S_{k}^{(2)}$ will be denoted briefly by $S_{k}$. We shall denote the diameter of any region $S_{k}$ by $R_{k}$; clearly $R_{k} \leq 3$. We also introduce the intervals

$$
J_{k}=\partial S_{k}^{(1)} \cap\{y=0\} .
$$

In $\S 4$ we have partitioned $\mathbb{R}_{+}^{2} \cap\{|x|<2,|y|<2\}$ into a (finite) sequence of rectangles (actually, squares) $Q_{n}$ whose smallest size is $\approx|z|^{-1 / 2}$. Here we shall need to partition, in a similar manner, each of the regions $S_{k}^{(2)}$ with $R_{k}>|z|^{-1 / 2}$. We begin with $L_{j}^{1}$.

We first divide $L_{j}^{1} \cap\left\{\frac{1}{2} \cdot \frac{3}{4} \leq y \leq \frac{3}{4}\right\}$ into two rectangles by the line $x=j+1$. Next we partition $L_{j}^{1} \cap\left\{\frac{1}{2^{2}} \cdot \frac{3}{4} \leq y \leq \frac{1}{2} \cdot \frac{3}{4}\right\}$ into four rectangles by the three lines

$$
x=j+\frac{1}{2}, x=j+1, x=j+\frac{3}{2}, \text { etc. }
$$

We proceed in this way until we arrive at

$$
L_{j}^{1} \cap\left\{\frac{1}{2^{l+1}} \cdot \frac{3}{4} \leq y \leq \frac{1}{2^{l}} \cdot \frac{3}{4}\right\}, \quad \text { where } \frac{1}{2^{l}} \approx|z|^{-1 / 2},
$$

which we partition into $2^{l+1}$ equal rectangles by lines $x=j+2 i / 2^{l+1}\left(1 \leq i \leq 2^{l+1}\right)$. Finally we partition also

$$
L_{j}^{1} \cap\left\{0 \leq y \leq \frac{1}{2^{l+1}} \cdot \frac{3}{4}\right\}
$$


into $2^{l+1}$ equal rectangles by the same lines $x=j+2 i / 2^{l+1}$.

Similarly we partition $L_{j}^{2}$, starting by dividing

$$
L_{j}^{2} \cap\left\{\frac{1}{2} \gamma_{j} \leq y \leq \gamma_{j}\right\}
$$

into two rectangles, etc. However we ensure that the line $y=\delta_{j}$ is a partition line: If

$$
\frac{1}{2^{h+1}} \gamma_{j}<\delta_{j}<\frac{1}{2^{h}} \gamma_{j}
$$

for some (nonnegative integer) $h$, then we replace both lines $y=\frac{1}{2^{h+1}} \gamma_{j}$ and $y=\frac{1}{2^{h}} \gamma_{j}$ by the line $y=\delta_{j}$. Thereafter we continue by partitioning

$$
L_{j}^{2} \cap\left\{\frac{1}{2} \delta_{j} \leq y \leq \delta_{j}\right\}
$$

into $2^{h+1}$ equal rectangles, etc. The process ends when we reach, as before, rectangles of size $\approx 1 /|z|^{1 / 2}$.

We finally turn to $L_{j}^{0}$. Here we take the $y$-lines to be

$$
\begin{aligned}
& y=\frac{1}{4}+\frac{1}{2} \cdot \frac{3}{4}, y=\frac{1}{4}+\left(\frac{1}{2}+\frac{1}{2^{2}}\right) \frac{3}{4}, \ldots, \\
& y=\frac{1}{4}+\left(\frac{1}{2}+\frac{1}{2^{2}}+\cdots+\frac{1}{2^{l+1}}\right) \frac{3}{4},
\end{aligned}
$$

where $1 / 2^{l+1} \approx 1 /|z|^{1 / 2}$, whereas the $x$-lines are defined in the same way as for $L_{j}^{1}$.

We denote by $Q_{n}$, or rather by $Q_{k n}$, the rectangles in the partition of $S_{k}^{(2)}$ and define

$$
q_{n}=q_{k n}=\text { center of } Q_{k n}, \quad d_{n}=d_{k n}=\text { diameter of } Q_{k n} .
$$

Then $d_{k n} \approx$ distance from $q_{k n}$ to either $\{y=0\}$ in case of $L_{j}^{1}$ or $\left.L_{j}^{2}\right)$ or $\{y=1\}$ in case of $L_{j}^{0}$. We define $\|\mu\|_{X_{2}}$ with respect to $S_{k}^{(2)}$ as in $\S 4$ and denote it for clarity by $\|\mu\|_{X_{2}\left(S_{k}^{(2)}\right)}$; thus

$$
\|\mu\|_{X_{2}\left(S_{k}^{(2)}\right)}=\sup _{n}\left\{d_{k n}\left(f_{Q_{k n}}|\mu|^{2}\right)^{1 / 2}\right\} .
$$

We also set

$$
\begin{aligned}
& \|\mu\|_{X_{2}}=\sup _{\substack{k \\
R_{k} \geq|z|^{-1 / 2}}}\left\{g\left(R_{k}, z\right) R_{k}^{3}\|\mu\|_{X_{2}\left(S_{k}^{(2)}\right)}\right\} \\
& =\sup _{\substack{k \\
R_{k} \geq|z|^{-1 / 2}}}\left\{g\left(R_{k}, z\right) R_{k}^{3} \sup _{n}\left\{d_{k n}\left(f_{Q_{k n}}|\mu|^{2}\right)^{1 / 2}\right\}\right\} .
\end{aligned}
$$

We define averages

$$
f_{S_{k}} h=\frac{1}{\left|S_{k}\right|} \int_{S_{k}} h, f_{J_{k}} f=\frac{1}{\left|J_{k}\right|} \int_{J_{k}} f .
$$


To each interval $J_{k}$ we associate a larger interval $\hat{J}_{k}$ of length $(1+\hat{\epsilon})\left|J_{k}\right|$, having the same center, where $\hat{\epsilon}<1$ and $\hat{\epsilon}$ is small enough so that $\hat{J}_{k}$ lies in $\{y=0,-\infty<$ $x<0\}$ and

$$
\operatorname{dist}\left(\hat{J}_{k},\{0\}\right) \geq \frac{1}{2} \operatorname{dist}\left(J_{k},\{0\}\right) .
$$

We introduce a cutoff function $\xi_{k}(x)$ in $\hat{J}_{k}$ such that $\left\{\xi_{k}=1\right\}$ contains the interval $J_{k}$ and

$$
\left|\partial_{x}^{m} \xi_{k}\right| \leq C\left|J_{k}\right|^{-m} \quad \text { for } 1 \leq m \leq 4
$$

Later on we shall use $L^{2}$ norms of fractional derivatives:

$$
\left[f_{\hat{J}_{k}}\left|\partial_{x}^{m}\left(\xi_{k} f\right)\right|^{2} d x\right]^{1 / 2} \quad\left(m=\frac{i}{2}, i=0,1,2, \ldots, 7\right)
$$

and denote them simply by

$$
\left[f_{J_{k}}\left|\partial_{x}^{m} f\right|^{2} d x\right]^{1 / 2}
$$

In the system (2.1)-(2.6), we shall refer to the data $Q \equiv(a, b, c, \mu)$ as a source, and introduce the norms

$$
\begin{aligned}
& \|Q\|_{X_{1}}=\|(a, b, c, \mu)\|_{X_{1}} \\
& =\sup _{k}\left\{g ( R _ { k } , z ) \left[R_{k}\left(f_{J_{k}}|a(x)|^{2}\right)^{1 / 2}+R_{k}^{\frac{5}{2}+1}\left(f_{J_{k}}\left|\partial_{x}^{5 / 2} a\right|^{2}\right)^{1 / 2}\right.\right. \\
& +R_{k}^{3}\left(f_{J_{k}}|b|^{2}\right)^{1 / 2}+R_{k}^{3+\frac{1}{2}}\left(f_{J_{k}}\left|\partial_{x}^{1 / 2} b\right|^{2}\right)^{1 / 2}+R_{k}^{3+\frac{1}{2}}\left(f_{J_{k}} \|\left.\left. z\right|^{1 / 4} b\right|^{2}\right)^{1 / 2} \\
& +R_{k}^{2}\left(f_{J_{k}}|c|^{2}\right)^{1 / 2}+R_{k}^{2+\frac{3}{2}}\left(f_{J_{k}}\left|\partial_{x}^{3 / 2} c\right|^{2}\right)^{1 / 2} \\
& \left.\left.+R_{k}^{2+\frac{3}{2}}\left(f \|\left.\left. z\right|^{3 / 4} c\right|^{2}\right)^{1 / 2}+R_{k}^{4}\left(f_{S_{k}^{(1)}}|\mu|^{2}\right)^{1 / 2}\right]\right\} \\
& \|Q\|_{X}=\|Q\|_{X_{1}}+\|\mu\|_{X_{2}} .
\end{aligned}
$$


We next define a norm for the solution $(W, F)$. It consists of three parts. The first part involves integrals taken over $S_{k}^{(1)}$ and $J_{k}$ :

$$
\begin{gathered}
\|(W, F)\|_{Y_{1}}=\max \left\{\operatorname { s u p } _ { k } \left[g ( R _ { k } , z ) \left[R_{k}^{4}\left(|z|+\frac{1}{R_{k}^{2}}\right)\left(f_{S_{k}^{(1)}}\left|\nabla^{2} W\right|^{2}\right)^{1 / 2}\right.\right.\right. \\
\left.\left.+R_{k}^{4}\left(f_{S_{k}^{(1)}}\left|\nabla^{4} W\right|^{2}\right)^{1 / 2}\right]\right] \\
\sup _{k}\left[g ( R _ { k } , z ) \left[R_{k}\left(|z|+\frac{1}{R_{k}}\right)\left(f_{J_{k}}|F-F(0)|^{2}\right)^{1 / 2}\right.\right. \\
\left.\left.\left.+R_{k}^{7 / 2}\left(f_{J_{k}}\left|\partial_{x}^{7 / 2}(F-F(0))\right|^{2}\right)^{1 / 2}\right]\right],|z|^{1 / 2}|F(0)|\right\} .
\end{gathered}
$$

The second part of the norm of $(W, F)$ consists of integrals taken over $S_{k}^{(2)}$ with $R_{k} \geq|z|^{-1 / 2}$ :

$$
\begin{aligned}
\|W\|_{Y_{2}}= & \sup _{\substack{k \\
R_{k} \geq|z|^{-1 / 2}}}\left\{g ( R _ { k } , z ) \operatorname { s u p } _ { n } \left[R_{k} d_{k n}^{3}\left(f_{Q_{k n}}\left|\nabla^{4} W\right|^{2}\right)^{1 / 2}\right.\right. \\
& \left.\left.+R_{k}^{3} d_{k n}|z|\left(f_{Q_{k n}}\left|\nabla^{2} W\right|^{2}\right)^{1 / 2}+|z| \frac{R_{k}^{3}}{\left|\log d_{k n}\right|}\left(f_{Q_{k n}}|\nabla W|^{2}\right)^{1 / 2}\right]\right\} .
\end{aligned}
$$

Finally, the third part of the norm of $(W, F)$ involves integrals over $S_{k}^{(2)}$ with $R_{k} \leq|z|^{-1 / 2}$ :

$$
\|W\|_{Y_{3}}=\sup _{\substack{R_{k} \leq|z|^{-1 / 2} \\ S_{k}}}\left\{g\left(R_{k}, z\right)\left[R_{k}^{2}\left(f_{S_{k}^{(2)}}\left|\nabla^{2} W\right|^{2}\right)^{1 / 2}+R_{k}^{4}\left(f_{S_{k}^{(2)}}\left|\nabla^{4} W\right|^{2}\right)^{1 / 2}\right]\right\} .
$$

We set

$$
\|(W, F)\|_{Y}=\|(W, F)\|_{Y_{1}}+\|W\|_{Y_{2}}+\|W\|_{Y_{3}} .
$$

We can now state the main a priori estimate: 
Lemma 5.1. If $(W, F)$ is a solution of (2.1)-(2.6) with $\operatorname{Re} z \geq 0,|z| \geq 2$, and if $W \in H^{2}(D)$ and $\|(W, F)\|_{Y}<\infty$, then

$$
\|(W, F)\|_{Y} \leq C|z|^{\frac{\epsilon}{2}+\frac{1}{2}}|\log | z||\|Q\|_{X},
$$

where $C$ is a constant independent of $z$ and of the source $Q$.

Remark 5.1. Since $W \in H^{2}(D)$ whereas $W_{x}(x, 1) \equiv 0$, we necessarily also have $W(x, 1) \equiv 0$.

Structure of the proof. Suppose the lemma is not true. Then there is a sequence

$$
\left(Q_{m}, F_{m}, W_{m}, z_{m}\right), \text { where } Q_{m}=\left(a_{m}, b_{m}, c_{m}, \mu_{m}\right),
$$

such that

$$
\left\|\left(W_{m}, F_{m}\right)\right\|_{Y} \gg\left|z_{m}\right|^{\frac{\epsilon}{2}+\frac{1}{2}}|\log | z_{m}||\left\|Q_{m}\right\|_{X},
$$

where " $\gg$ " means that the left-hand side divided by the right-hand side converges to $\infty$ as $m \rightarrow \infty$. We wish to derive a contradiction. To do that we may assume that $\left\|\left(W_{m}, F_{m}\right)\right\|_{Y}=1$. We shall then show (i) that the sequence $\left(W_{m}, F_{m}\right)$ (or a scaled version of it) converges in some weak norm to a solution $(\varphi, f)$ of a homogeneous elliptic system whose only solution is zero, and (ii) that the sequence $\left(W_{m}, F_{m}\right)$ actually converges to zero in the stronger norm $\left\|\left(W_{m}, F_{m}\right)\right\|_{Y}$.

Consider first the situation where

$$
\left\|\left(W_{m}, F_{m}\right)\right\|_{Y_{1}} \text { is the dominant term in }\|(W, F)\|_{Y},
$$

i.e.

$$
\left\|\left(W_{m}, F_{m}\right)\right\|_{Y_{1}} /\left[\left\|W_{m}\right\|_{Y_{2}}+\left\|W_{m}\right\|_{Y_{3}}\right] \rightarrow \infty \text { as } m \rightarrow \infty .
$$

We subdivide it into three cases:

$$
\begin{gathered}
\sup _{k}\left[g ( R _ { k } , z _ { m } ) \left[R_{k}^{4}\left(\left|z_{m}\right|+\frac{1}{R_{k}^{2}}\right)\left(f_{S_{k}^{(1)}}\left|\nabla^{2} W_{m}\right|^{2}\right)^{1 / 2}\right.\right. \\
\left.\left.+R_{k}^{4}\left(f_{S_{k}^{(1)}}\left|\nabla^{4} W_{m}\right|^{2}\right)^{1 / 2}\right]\right] \\
\geq c\left\|\left(W_{m}, F_{m}\right)\right\|_{Y}
\end{gathered}
$$

for some positive constant $c$;

$$
\left|z_{m}\right|^{1 / 2}\left|F_{m}(0)\right| \geq c\left\|\left(W_{m}, F_{m}\right)\right\|_{Y}
$$

where $c$ is some positive constant; and 
both (5.13) and (5.14) do not hold, so that

$$
\begin{aligned}
& \sup _{k}\left[g ( R _ { k } , z _ { m } ) \left[R_{k}\left(\left|z_{m}\right|+\frac{1}{R_{k}}\right)\left(f_{J_{k}}\left|F_{m}-F_{m}(0)\right|^{2}\right)^{1 / 2}\right.\right. \\
& \left.\left.+R_{k}^{7 / 2}\left(f_{J_{k}}\left|\partial_{x}^{7 / 2}\left(F_{m}-F_{m}(0)\right)\right|^{2}\right)^{1 / 2}\right]\right] \geq c\left\|\left(W_{m}, F_{m}\right)\right\|_{Y},
\end{aligned}
$$

where $c$ is a positive constant and, in addition,

$$
\left[\operatorname{LHS} \text { of (5.15)] } \gg\left\{[\operatorname{LHS} \text { of }(5.13)]+\left|z_{m}\right|^{1 / 2}\left|F_{m}(0)\right|\right\},\right.
$$

where LHS means left-hand side.

In addition to the case where (5.12) holds we also need to consider the cases where either $\left\|W_{m}\right\|_{Y_{2}}$ or $\left\|W_{m}\right\|_{Y_{3}}$ is the dominant term, i.e.,

$$
\begin{gathered}
\sup _{\substack{k \\
R_{k} \geq|z|^{-1 / 2}}}\left\{g ( R _ { k } , z _ { m } ) \operatorname { s u p } _ { n } \left[R_{k} d_{k n}^{3}\left(f_{Q_{k n}}\left|\nabla^{4} W_{m}\right|^{2}\right)^{1 / 2}\right.\right. \\
+R_{k}^{3} d_{k n}\left|z_{m}\right|\left(f_{Q_{k n}}\left|\nabla^{2} W_{m}\right|^{2}\right)^{1 / 2} \\
\left.\left.+\left|z_{m}\right| \frac{R_{k}^{3}}{\left|\log d_{k n}\right|}\left(f_{Q_{k n}}\left|\nabla W_{m}\right|^{2}\right)^{1 / 2}\right]\right\} \geq c\left\|\left(W_{m}, F_{m}\right)\right\|_{Y}
\end{gathered}
$$

or

$$
\begin{aligned}
\sup _{\substack{k \\
R_{k} \leq|z|^{-1 / 2}}}\left\{g\left(R_{k}, z_{m}\right)\right. & {\left[R_{k}^{2}\left(f_{S_{k}^{(2)}}\left|\nabla^{2} W_{m}\right|^{2}\right)^{1 / 2}\right.} \\
& \left.\left.+R_{k}^{4}\left(f_{S_{k}^{(2)}}\left|\nabla^{4} W_{m}\right|^{2}\right)^{1 / 2}\right]\right\} \geq c\left\|\left(W_{m}, F_{m}\right)\right\|_{Y},
\end{aligned}
$$

where $c$ is a positive constant.

In each of the above cases we may assume that the "sup" is attained at some $k=k_{m}$; for if the supremum is not attained, we simply choose $k=k_{m}$ such that it realizes a value larger than, say, $\frac{1}{2} \sup _{k}$, and this will not change any of the subsequent arguments. 
In all the cases where

$$
\limsup _{m \rightarrow \infty} R_{k_{m}}\left|z_{m}\right|^{1 / 2}<\infty
$$

we can derive a contradiction by reducing the situation (sometimes after using a blow-up argument) to a uniqueness statement and then apply a Liouville type theorem (such as one of the theorems established in [7]). However in the case

$$
R_{k_{m}}\left|z_{m}\right|^{1 / 2} \rightarrow \infty
$$

we do not have uniqueness. This case requires a much more refined analysis which involves deriving uniform estimates for the sequence $\left(W_{m}, F_{m}\right)$ for all large $m$. This additional analysis will be given in Sections 14, 15 .

For simplicity we shall first consider the case

$$
R_{k_{m}} \approx 1
$$

this is carried out in $\S \S 6,7$ (and, partially, in the Appendix). The case $R_{k_{m}} \rightarrow 0$ is analyzed in $\S \S 8-10$; we shall consider there separately the cases

$$
R_{k_{m}}\left|z_{m}\right| \rightarrow\left\{\begin{array}{l}
0, \\
\text { finite number } \neq 0, \\
\infty,
\end{array}\right.
$$

and the last subcase will further be subdivided according to whether $R_{k_{m}}^{2}\left|z_{m}\right| \rightarrow \infty$, 0 or a finite number $\omega \neq 0$.

Remark 5.2. In analyzing the various cases which occur in the proof of Lemma 5.1 we shall not always require the full strength of (5.11). In some cases it will suffice to assume only that

$$
\left\|\left(W_{m}, F_{m}\right)\right\|_{Y} \gg\left|z_{m}\right|^{\frac{\epsilon}{2}}|\log | z_{m}||\left\|Q_{m}\right\|_{X}
$$

or even just that

$$
\left\|\left(W_{m}, F_{m}\right)\right\|_{Y} \gg\left\|Q_{m}\right\|_{X},
$$

in order to derive a contradiction.

$$
\text { 6. The CASE } R_{k_{m}} \approx 1 \text { WITH (5.13) }
$$

We divide this case into two subcases:

$$
\begin{aligned}
& g\left(R_{k_{m}}, z_{m}\right) R_{k_{m}}^{4}\left(f_{S_{k_{m}}^{(1)}}\left|\nabla^{4} W_{m}\right|^{2}\right)^{1 / 2} \\
& \geq \sup _{k}\left[g\left(R_{k}, z_{m}\right) R_{k}^{4}\left(\left|z_{m}\right|+\frac{1}{R_{k}^{2}}\right)\left(f_{S_{k}^{(1)}}\left|\nabla^{2} W_{m}\right|^{2}\right)^{1 / 2}\right],
\end{aligned}
$$


and

$$
\begin{gathered}
\sup _{k}\left[g\left(R_{k}, z_{m}\right) R_{k}^{4}\left(f_{S_{k}^{(1)}}\left|\nabla^{4} W_{m}\right|^{2}\right)^{1 / 2}\right] \\
\quad \leq 2 g\left(R_{k_{m}}, z_{m}\right) R_{k_{m}}^{4}\left(\left|z_{m}\right|+\frac{1}{R_{k_{m}}^{2}}\right)\left(f_{S_{k_{m}}^{(1)}}\left|\nabla^{2} W_{m}\right|^{2}\right)^{1 / 2} .
\end{gathered}
$$

Since $\left|z_{m}\right| \geq 2$ and $R_{k_{m}} \approx 1$, these subcases reduce to

$$
\left(f_{S_{k_{m}}^{(1)}}\left|\nabla^{4} W_{m}\right|^{2}\right)^{1 / 2} \geq c\left(f_{S_{k_{m}}^{(1)}}\left|\nabla^{2} W_{m}\right|^{2}\right)^{1 / 2}
$$

and

$$
\left(f_{S_{k_{m}}^{(1)}}\left|\nabla^{4} W_{m}\right|^{2}\right)^{1 / 2} \leq C\left(f_{S_{k_{m}}^{(1)}}\left|\nabla^{2} W_{m}\right|^{2}\right)^{1 / 2},
$$

respectively, when $c$ and $C$ are positive constants. We shall first consider the case (6.3)

We may assume that either

$$
\left|z_{m}\right| \rightarrow \infty
$$

or

$$
z_{m} \rightarrow z_{*} \quad\left(z_{*} \text { finite, } \quad\left|z_{*}\right| \geq 2\right)
$$

and consider first the case (6.5). Introduce the functions

$$
\widetilde{W}_{m}=\frac{W_{m}}{\left(f_{S_{k_{m}}^{(1)}}\left|\nabla^{4} W_{m}\right|^{2}\right)^{1 / 2}}, \quad \widetilde{F}_{m}=\frac{F_{m}}{\left(f_{S_{k_{m}}^{(1)}}\left|\nabla^{4} W_{m}\right|^{2}\right)^{1 / 2}}
$$

and the sources

$$
\widetilde{Q}_{m}=\left(\widetilde{a}_{m}, \widetilde{b}_{m}, \widetilde{c}_{m}, \widetilde{\mu}_{m}\right)
$$

where

$$
\widetilde{Q}_{m}=\frac{Q_{m}}{\left(f_{S_{k_{m}}^{(1)}}\left|\nabla^{4} W_{m}\right|^{2}\right)^{1 / 2}}
$$


Then

$$
\left(f_{S_{k_{m}}^{(1)}}\left|\nabla^{4} \widetilde{W}_{m}\right|^{2}\right)^{1 / 2}=1
$$

and

$$
\left(f_{S_{k_{m}}^{(1)}}\left|\nabla^{2} \widetilde{W}_{m}\right|^{2}\right)^{1 / 2} \leq \frac{1}{\left|z_{m}\right|} .
$$

Further,

$$
z_{m} \Delta \widetilde{W}_{m}-\Delta^{2} \widetilde{W}_{m}=\widetilde{\mu}_{m} \quad \text { in } D
$$

and, by (5.23),

$$
\int_{S_{k_{m}}^{(1)}}\left|\widetilde{\mu}_{m}\right|^{2} \rightarrow 0
$$

The functions $\widetilde{W}_{m}, \widetilde{F}_{m}$ satisfy $(2.2)-(2.6)$ with $a=\widetilde{a}_{m}, b=\widetilde{b}_{m}, c=\widetilde{c}_{m}$. By the definition of $\left\|Q_{m}\right\|_{X}$ and (5.23),

$$
\begin{gathered}
f_{J_{k_{m}}}\left|\widetilde{a}_{m}(x)\right|^{2} \rightarrow 0, \quad f_{J_{k_{m}}}\left|\partial_{x}^{5 / 2} \widetilde{a}_{m}\right|^{2} \rightarrow 0, \quad f_{J_{k_{m}}}\left|\partial_{x}^{1 / 2} \widetilde{b}_{m}\right|^{2} \rightarrow 0, \\
\left|z_{m}\right|^{1 / 2} f_{J_{k_{m}}}\left|\widetilde{b}_{m}\right|^{2} \rightarrow 0, \quad \int_{J_{k_{m}}}\left|\widetilde{c}_{m}\right|^{2} \rightarrow 0, \\
f_{J_{k_{m}}}\left|\partial_{x}^{3 / 2} \widetilde{c}_{m}\right|^{2} \rightarrow 0, \quad\left|z_{m}\right|^{3 / 2} f_{J_{k_{m}}}\left|\widetilde{c}_{m}\right|^{2} \rightarrow 0 .
\end{gathered}
$$

Multiplying (6.10) by a function $\varphi \in C_{0}^{\infty}(D)$ and integrating, we get

$$
\int\left(z_{m} \widetilde{W}_{m} \Delta \varphi-\widetilde{W}_{m} \Delta^{2} \varphi\right)=\int \widetilde{\mu}_{m} \varphi
$$

Now, since

$$
\left\|\widetilde{W}_{m}\right\|_{Y_{2}} \leq C
$$

we have, for any $S_{k}^{(2)}$ of the form $L_{j}^{0}$,

$$
\left(f_{Q_{k n}}\left|z_{m} \nabla \widetilde{W}_{m}\right|^{2}\right)^{1 / 2} \leq C\left|\log d_{k n}\right| .
$$

Recalling (by Remark 5.1) that $\widetilde{W}_{m}(x, 1)=0$, we deduce that

$$
\left(f_{Q_{k n}}\left|z_{m} \widetilde{W}_{m}\right|^{2}\right)^{1 / 2} \leq C d_{k n}\left|\log d_{k n}\right|,
$$


i.e.,

$$
\left|z_{m} \widetilde{W}_{m}(x, y)\right| \leq C(1-y)|\log (1-y)|
$$

in average. A similar estimate can be derived with respect to the $L_{j}^{1}$, yielding

$$
\left|z_{m} \widetilde{W}_{m}(x, y)\right| \leq C_{\epsilon_{0}} y|\log y|
$$

in average, for $x \geq \epsilon_{0}\left(\epsilon_{0}\right.$ any small positive number).

Consider first the special case where

$$
\text { all the } S_{k_{m}}^{(1)} \text { coincide with a specific } S_{j_{0}}^{(1)} \text {. }
$$

Using the above estimate and the estimates on the $L^{2}$-norms of $\nabla^{2} \widetilde{W}_{m}$ which follow from the fact that $\left\|\left(\widetilde{W}_{m}, \widetilde{F}_{m}\right)\right\|_{Y} \leq C$, we find that

$$
\begin{aligned}
& z_{m} \widetilde{W}_{m} \rightarrow G \text { weakly in } H^{2}\left(D_{0}\right), \\
& z_{m} \widetilde{W}_{m} \rightarrow G \text { strongly in } H^{3 / 2}\left(D_{0}\right)
\end{aligned}
$$

for any compact domain $D_{0}$ in $D \cup\{(x, 0) ; x<0\}$. Taking $m \rightarrow \infty$ in (6.13) we find that

$$
\Delta G=0 \text { in } D .
$$

Further, $G$ is uniformly bounded away from the origin,

$$
\begin{aligned}
& G(x, 1)=0 \text { if }-\infty<x<\infty, \\
& G(x, 0)=0 \text { if } 0<x<\infty,
\end{aligned}
$$

and, by the argument in $[7, \S 7]$ which leads to $[7,(7.12)]$,

$$
G_{y}(x, 0)=0 \text { if } x<0 .
$$

We also have the estimate

$$
R_{k}^{4}\left(f_{S_{k}^{(1)}}\left|\nabla^{2} G\right|^{2}\right)^{1 / 2}+R_{k}^{3} d_{k n}\left(f_{S_{k}^{(2)}}\left|\nabla^{2} G\right|^{2}\right)^{1 / 2} \leq C R_{k}^{\frac{1}{2}-\epsilon}
$$

for all the $S_{k}^{(1)}, S_{k}^{(2)}$ near the origin. This estimate implies that $G$ has the expansion

$$
G(x, y)=A r^{-3 / 2} \sin \frac{3 \theta}{2}+B r^{-1 / 2} \sin \frac{\theta}{2}+O\left(r^{1 / 2}\right)
$$

near the origin.

Let $G_{0}$ and $G_{1}$ be the bounded harmonic functions in $D$ satisfying

$$
\begin{aligned}
& G_{0}=0 \text { if } y=1 \text { or } y=0, x>0, \\
& \partial_{y} G_{0}=0 \text { if } y=0, x<0, \\
& G_{0}=r^{-3 / 2} \sin \frac{3 \theta}{2}+o(1) \text { if } r \rightarrow 0
\end{aligned}
$$

and

$$
\begin{aligned}
& G_{1}=0 \text { if } y=1 \text { or } y=0, x>0, \\
& \partial_{y} G_{1}=0 \text { if } y=0, x<0 \\
& G_{1}=r^{-1 / 2} \sin \frac{\theta}{2}+o(1) \text { if } r \rightarrow 0 .
\end{aligned}
$$


By comparing $G_{i}$ with

$$
\epsilon \log \left(x^{2}+y^{2}\right)+A\left(2 y-y^{2}\right) c^{-\sqrt{2} x} \quad \text { for } x>1
$$

and with

$$
\epsilon \log \left(x^{2}+y^{2}\right)+A\left(2(y+1)-(y+1)^{2}\right) e^{\sqrt{2} x} \quad \text { for } x<-1
$$

with $A$ a sufficiently large constant and $\epsilon$ an arbitrarily small positive constant, we find that

$$
G_{0} \rightarrow 0, G_{1} \rightarrow 0 \text { exponentially fast, as }|x| \rightarrow \infty .
$$

By the maximum principle,

$$
G-A G_{0}-B G_{1}=0
$$

Lemma 6.1. $A=0$ and $B=0$, so that $G \equiv 0$.

The proof is given in $\S \S 14,15$.

Remark 6.1. The proof of Lemma 6.1 requires the full use of the inequality (5.11).

From (6.16) and Lemma 6.1 it follows that

$$
\left|z_{m}\right|\left(f_{S_{k_{m}}^{(1)}}\left|\nabla^{j} \widetilde{W}_{m}\right|^{2}\right)^{1 / 2} \rightarrow 0 \quad\left(j=0,1, \frac{3}{2}\right) .
$$

Introduce a cutoff function $\eta$ in $S_{k_{m}}^{(1)}$ such that $\eta=0$ in some $\epsilon_{1}$-neighborhood of $\partial S_{k_{m}}^{(1)} \cap\{y>0\}, \eta=1$ outside a $\left(2 \epsilon_{1}\right)$-neighborhood of $\partial S_{k_{m}}^{(1)} \cap\{y>0\}$, and

$$
\frac{\partial^{j} \eta}{\partial y^{i}}=0 \text { on } y=0,-\infty<x<0(1 \leq j \leq 3) \text {. }
$$

Define

$$
\hat{W}_{m}=\eta \widetilde{W}_{m}
$$

Then

$$
z_{m} \Delta \hat{W}_{m}-\Delta^{2} \hat{W}_{m}=\hat{\mu}_{m} \quad \text { in } D
$$

where

$$
\hat{\mu}_{m}=\eta \widetilde{\mu}_{m}+\hat{H}\left(\partial^{3} \widetilde{W}_{m}, \partial^{2} \widetilde{W}_{m}, \partial \widetilde{W}_{m}, \widetilde{W}_{m}, z_{m} \partial \widetilde{W}_{m}, z_{m} \widetilde{W}_{m}\right)
$$

and $\hat{H}$ is a linear function of the indicated variables. By interpolation, for any $\epsilon>0$,

$$
\left\|\partial^{3} \widetilde{W}_{m}\right\|_{L^{2}\left(S_{k_{m}}^{(1)}\right)} \leq \epsilon\left\|\nabla^{4} \widetilde{W}_{m}\right\|_{L^{2}\left(S_{k_{m}}^{(1)}\right)}+C_{\epsilon}\left\|\widetilde{W}_{m}\right\|_{L^{2}\left(S_{k_{m}}^{(1)}\right)} .
$$

Recalling (6.20) and (6.8), we conclude that

$$
\left\|\partial^{3} \widetilde{W}_{m}\right\|_{L^{2}\left(S_{k_{m}}^{(1)}\right)} \leq C \epsilon
$$

if $m$ is large enough. The same can be proved for each of the terms in $\hat{H}$. Hence

$$
\|\hat{\mu}\|_{L^{2}\left(S_{k_{m}}^{(1)}\right)} \rightarrow 0 \text { if } m \rightarrow \infty .
$$


We now turn to the boundary conditions of $\hat{W}_{m}$ on $y=0 . \hat{W}_{m}$ satisfies (2.3) and (2.4)-(2.6) with $a=\hat{a}_{m}, b=\hat{b}_{m}, c=\hat{c}_{m}$. We want to show that

$$
\partial_{x}^{5 / 2} \hat{a}_{m}, \hat{a}_{m}, \partial_{x}^{1 / 2} \hat{b}_{m},\left|z_{m}\right|^{1 / 4} \hat{b}_{m}, \hat{c}_{m}, \partial_{x}^{3 / 2} \hat{c}_{m},\left|z_{m}\right|^{3 / 4} \hat{c}_{m} \rightarrow 0 \text { in } L^{2} .
$$

We begin with

$$
\hat{a}_{m}=\eta \widetilde{a}_{m}+\beta_{0} \widetilde{F}_{m} \partial_{x} \eta-\widetilde{W}_{m} \partial_{x} \eta
$$

Because of (5.12),

$$
\left|z_{m}\right|\left(f_{J_{k_{m}}}\left|\widetilde{F}_{m}-\widetilde{F}_{m}(0)\right|^{2}\right)^{1 / 2} \leq C, \quad\left(f_{J_{k_{m}}}\left|\partial_{x}^{7 / 2}\left(\widetilde{F}_{m}-\widetilde{F}_{m}(0)\right)\right|^{2}\right)^{1 / 2} \leq C,
$$

and then, by interpolation,

$$
\left(f_{J_{k_{m}}}\left|\partial_{x}^{j}\left(\widetilde{F}_{m}-\widetilde{F}_{m}(0)\right)\right|^{2}\right)^{1 / 2} \rightarrow 0 \text { for } 0 \leq j \leq 3
$$

By (6.20), (6.8) and interpolation [9, p. 49] we also have

$$
\int_{S_{k_{m}}^{(1)}}\left|\nabla^{3+1 / 2} \widetilde{W}_{m}\right|^{2} \rightarrow 0
$$

and, therefore, by the trace theorem,

$$
f_{J_{k_{m}}}\left|\nabla^{j} \widetilde{W}_{m}\right|^{2} \rightarrow 0 \text { for }|j| \leq 3 .
$$

Combining (6.26) (with $\widetilde{F}_{m}(0) \rightarrow 0$, which follows from (5.6), (5.13) and (6.5)), (6.28) and recalling (6.12), we conclude that

$$
f_{J_{k_{m}}}\left|\partial_{x}^{5 / 2} \hat{a}_{m}\right|^{2} \rightarrow 0 \text { if } m \rightarrow \infty .
$$

The proof that $\hat{a}_{m} \rightarrow 0$ in $L^{2}\left(J_{k_{m}}\right)$ is similar.

Consider next $\hat{b}_{m}$. Using (6.21) we find that

$$
\begin{gathered}
\hat{b}_{m}=\eta \hat{b}_{m}-3 \frac{\partial \widetilde{W}_{m}}{\partial y} \partial_{x}^{3} \eta-6 \frac{\partial \widetilde{W}_{m}}{\partial x \partial y} \partial_{x} \eta-\sigma W_{m} \partial_{x}^{3} \eta \\
-3 \sigma \frac{\partial \widetilde{W}_{m}}{\partial x} \partial_{x}^{2} \eta-3 \sigma \frac{\partial^{2} W_{m}}{\partial x^{2}} \partial_{x} \eta .
\end{gathered}
$$

In view of (6.12), (6.28),

$$
f_{J_{k_{m}}}\left|\partial_{x}^{1 / 2} \hat{b}_{m}\right|^{2} \rightarrow 0
$$

Also, by (6.20),

$$
\left|z_{m}\right|\left\|\hat{W}_{m}\right\|_{H^{3 / 2}\left(S_{k_{m}}^{(1)}\right)} \rightarrow 0
$$


and, therefore, by the trace theorem,

$$
\left|z_{m}\right| f_{J_{k_{m}}}\left|\partial_{x} \hat{W}_{m}\right|^{2} \rightarrow 0 .
$$

Applying the interpolation inequality [9, p. 49]

$$
\left(\int_{\mathbb{R}^{2}}\left|D^{1 / 2} w\right|^{2}\right)^{1 / 2} \leq C\left(\int_{\mathbb{R}^{2}}\left(w^{2}+\left|D^{2} w\right|^{2}\right)\right)^{1 / 8}\left(\int_{\mathbb{R}^{2}}|w|^{2}\right)^{3 / 8}
$$

to $w=\nabla^{2} \widetilde{W}_{m}$ and using (6.8), (6.9), we get

$$
\left|z_{m}\right|^{3 / 4}\left\|\nabla^{2+1 / 2} W_{m}\right\|_{L^{2}\left(S_{k_{m}}^{(1)}\right)} \leq C
$$

and, by the trace theorem,

$$
\left|z_{m}\right|^{3 / 4}\left\|\widetilde{W}_{m}\right\|_{H^{2}\left(J_{k_{m}}\right)} \leq C .
$$

Combining this with (6.30) and recalling (6.12), we find that

$$
\left|z_{m}\right|^{1 / 4}\left(f_{J_{k_{m}}}\left|\hat{b}_{m}\right|^{2}\right)^{1 / 2} \rightarrow 0
$$

We finally consider

$$
\hat{c}_{m}=\eta \widetilde{c}_{m}-\widetilde{W}_{m} \partial_{x}^{2} \eta-2 \frac{\partial \widetilde{W}_{m}}{\partial x} \cdot \partial_{x} \eta
$$

Using (6.28), (6.31) and recalling (6.12), we immediately deduce that

$$
f_{J_{k_{m}}}\left|\partial_{x}^{3 / 2} \hat{c}_{m}\right|^{2} \rightarrow 0, \quad\left|z_{m}\right|^{3 / 2} f_{J_{k_{m}}}\left|\hat{c}_{m}\right|^{2} \rightarrow 0,
$$

and since $\left|z_{m}\right| \geq 2$, also $\hat{c}_{m} \rightarrow 0$ in $L^{2}\left(J_{k m}\right)$. This completes the proof of $(6.25)$. We are now in a position where we can apply Lemma 4.3 to deduce that

$$
f_{S_{k_{m}}^{(1)}}\left|\nabla^{4} \hat{W}_{m}\right|^{2} \rightarrow 0, \quad \text { i.e., } \quad \int_{S_{k_{m}}^{(1)}}\left|\nabla^{4}\left(\eta \widetilde{W}_{m}\right)\right|^{2} \rightarrow 0 .
$$

The proof of (6.32) can be carried out not only for $S_{k_{m}}^{(1)}$ but also for the union $\bigcup_{j} S_{j}$, where the $j$ 's are such that $S_{j} \cap S_{k_{m}}^{(1)} \neq \phi$. We can choose, in this case, the $\eta$ to be such that $\eta=1$ in $S_{k_{m}}^{(1)}$. Consequently

$$
f_{S_{k_{m}}^{(1)}}\left|\nabla^{4} \widetilde{W}_{m}\right|^{2} \rightarrow 0 \quad \text { if } m \rightarrow \infty,
$$

which is a contradiction to (6.8).

In the above proof it was assumed that (6.15) holds. If this is not the case then (by taking a subsequence) we may assume that the $S_{k_{m}}^{(1)}$ converge to $x=-\infty$ as $m \rightarrow \infty$. We then work with translates of $\widetilde{W}_{m}$ :

$$
\widetilde{\widetilde{W}}_{m}(x, y)=\widetilde{W}_{m}\left(x-\tau_{m}, y\right) \quad\left(\tau_{m} \rightarrow \infty\right) \text {. }
$$


The limit $G$ of the $z_{m} \widetilde{\widetilde{W}}_{m}$ is a bounded harmonic function in $D$, satisfying

$$
G(x, y)=0, \quad G_{y}(x, 0)=0 \quad \text { for all } x \in \mathbb{R}^{1} .
$$

Hence $G \equiv 0$, and we can then proceed as before to derive a contradiction.

So far we have assumed that (6.5) holds. Consider next the case (6.6). Using (6.9) and the boundary conditions $\widetilde{W}_{m}=\partial_{y} \widetilde{W}_{m}=0$ at $y=1$, we deduce, by Poincaré's inequality, that

$$
\left|\widetilde{W}_{m}\right| \leq C \text { in } D .
$$

Further, since $R_{k}<\left|z_{m}\right|^{-1 / 2}$ for $R_{k}$ small and for all $m$,

$$
g\left(R_{k}, z_{m}\right) R_{k}^{2}\left(f_{S_{k}}\left|\nabla^{2} \widetilde{W}_{m}\right|^{2}\right)^{1 / 2} \leq C
$$

for all $S_{k}$ near the origin. By applying the Poincaré inequality to

$$
\widetilde{W}_{m}\left(R_{k} x, R_{k} y\right) / R_{k}^{\frac{3}{2}-\epsilon}
$$

in $\left\{y>0,1<x^{2}+y^{2}<2\right\}$ and using (6.34) we deduce that

$$
\left|\widetilde{W}_{m}\right| \leq C r^{\frac{3}{2}-\epsilon} \text { near the origin. }
$$

We can now use elliptic estimates to conclude that

$$
\widetilde{W}_{m} \rightarrow K \text { weakly in } L_{\text {loc }}^{2}(D)
$$

and

$$
z_{*} \Delta K-\Delta^{2} K=0 \text { in } D .
$$

$K$ also satisfies the homogeneous boundary conditions of (2.2)-(2.6) in a weak sense and, by [9] (as in $\S 3$ ), also in the classical sense. From (6.35) we get the same bound for $K$ and then, by the regularity results established in [5] and [6],

$$
D^{\sigma} K=O\left(r^{\frac{3}{2}-|\sigma|}\right)
$$

for $|\sigma| \leq 3$. Since $K$ is bounded in $D$, we can use the Phragmén-Lindelöf theorem of Lax [8] (see also [6, Lemma 7.2]) to deduce that $K$ decays exponentially fast in $D$ as $|x| \rightarrow \infty$; by elliptic estimates we get the same decay for derivatives $D^{\sigma} K$.

To obtain the appropriate decay as $x \rightarrow-\infty$ and prove that $K \equiv 0$ we multiply (6.36) by $\bar{K}$, the complex conjugate of $K$, integrate over $D \cap\{-M<x<\infty\} \equiv D_{M}$, and perform integration by parts. We deduce (cf. the proof of Theorem 3.4) that

$$
\begin{aligned}
\int_{D_{M}} & {\left[\left|\frac{\partial^{2} K}{\partial x^{2}}-\frac{\partial^{2} K}{\partial y^{2}}\right|^{2}+4\left|\frac{\partial^{2} K}{\partial x \partial y}\right|^{2}\right]+\left(\operatorname{Re} z_{*}\right) \int_{D_{m}}|\nabla K|^{2} } \\
& +\sigma\left(\operatorname{Re} z_{*}\right) \int_{-M}^{0}\left|\frac{\partial F}{\partial x}\right|^{2} d x+\frac{1}{2} \sigma \beta_{0}\left|\frac{\partial F(0)}{\partial x}\right|^{2}=I_{M}+\frac{1}{2} \sigma \beta_{0}\left|\frac{\partial F(-M)}{\partial x}\right|^{2}
\end{aligned}
$$


where $I_{M}$ is the integral of (linear combination of) products $D^{i} K \cdot D^{j} \bar{K}$ taken over $l_{M}=D \cap\{x=-M\}$, where $i \geq 0, j \geq 0, i+j \leq 3$. Since

$$
f_{S_{k}^{(1)}}\left[\left|\nabla^{2} \widetilde{W}_{m}\right|^{2}+\left|\nabla^{4} \widetilde{W}_{m}\right|^{2}\right] \leq C \quad \text { for all } k, m,
$$

this bound holds also for $K$ (and all $k$ ). Hence $I_{M}$ remains bounded for a sequence $M=M_{n} \rightarrow \infty$. By (5.13), also $|\partial F(-M) / \partial x|$ remains bounded as $M \rightarrow \infty$. But then the left-hand side of (6.37) must remain bounded as $M \rightarrow \infty$. It follows that $D^{2} K$ belongs to $D$ and, by (6.36) and elliptic estimates [1], [2] (cf. [7, Remark 10.2]) all the derivatives of $K$ of orders $\leq 4$ belong to $L^{2}\{D \cap(-\infty<x<-1)\}$ and $F_{x}(-M) \rightarrow 0$ if $M \rightarrow \infty$. Consequently

$$
I_{M} \rightarrow 0 \quad \text { for a sequence } M \rightarrow \infty
$$

and thus the left-hand side of (6.37) converges to zero as $M \rightarrow \infty$. It follows that $K=0$. Consequently

$$
f_{S_{k_{m}}^{(1)}}\left|\widetilde{W}_{m}\right|^{2} \rightarrow 0
$$

By (6.8) and interpolation we then deduce that (6.27) holds and therefore also (6.28). This enables us to proceed as before and establish (6.32) and (6.33), thus contradicting (6.8). We have thus completed the proof in case (6.3) holds.

We next consider the case (6.4), and define

$$
\widetilde{W}_{m}=\frac{W_{m}}{\left|z_{m}\right|\left(f_{S_{k_{m}}^{(1)}}\left|\nabla^{2} W_{m}\right|^{2}\right)^{1 / 2}}
$$

Then

$$
\left|z_{m}\right|\left(f_{S_{k_{m}}^{(1)}}\left|\nabla^{2} \widetilde{W}_{m}\right|^{2}\right)^{1 / 2}=1
$$

$$
\left(f_{S_{k_{m}}^{(1)}}\left|\nabla^{4} \widetilde{W}_{m}\right|^{2}\right)^{1 / 2} \leq 1
$$

and

$$
z_{m} \Delta \widetilde{W}_{m}-\Delta^{2} \widetilde{W}_{m}=\widetilde{\mu}_{m}, \quad \int_{S_{k_{m}}^{(1)}}\left|\widetilde{\mu}_{m}\right|^{2} \rightarrow 0 .
$$

Consider first the case (6.5). We can proceed as before to deduce that

$$
f_{S_{k_{m}}^{(1)}}\left|\nabla^{4} \widetilde{W}_{m}\right|^{2} \rightarrow 0
$$


and similarly that

$$
\left|z_{m}\right| f_{S_{k_{m}}^{(1)}}\left|\nabla^{2} \widetilde{W}_{m}\right|^{2} \rightarrow 0
$$

which is a contradiction to $(6.39)$.

If $z_{m}$ satisfies (6.6) then $\widetilde{W}_{m} \rightarrow K$ weakly in $L_{\text {loc }}^{2}(D)$, and

$$
z_{*} \Delta K-\Delta^{2} K=0 \text { in } D \text {; }
$$

$K$ also satisfies the homogeneous boundary conditions (of (2.2)-(2.6)) in the weak sense. As before we deduce that $K \equiv 0$, and we can then proceed as above to derive the relation

$$
\left|z_{m}\right| \int_{S_{k_{m}}^{(1)}}\left|\nabla^{2} \widetilde{W}_{m}\right|^{2} \rightarrow 0 \text { as } m \rightarrow \infty
$$

which is a contradiction to (6.39).

7. The CASE $R_{k_{m}} \approx 1$ With $(5.15),(5.16)$

We shall consider separately the subcases

$$
\begin{aligned}
& \sup _{k}\left(f_{J_{k}}\left|F_{m}-F_{m}(0)\right|^{2}\right)^{1 / 2}\left|z_{m}\right| \geq \sup _{k}\left(f_{J_{k}}\left|\partial_{x}^{7 / 2}\left(F_{m}-F_{m}(0)\right)\right|^{2}\right)^{1 / 2}, \\
& \sup _{k}\left(f_{J_{k}}\left|F_{m}-F_{m}(0)\right|^{2}\right)\left|z_{m}\right| \leq \sup _{k}\left(f_{J_{k}}\left|\partial_{x}^{7 / 2}\left(F_{m}-F_{m}(0)\right)\right|^{2}\right)^{1 / 2} .
\end{aligned}
$$

We begin with (7.1). Introduce

$$
\widetilde{W}_{m}=\frac{W_{m}}{\left(f_{J_{k_{m}}}\left|F_{m}-F_{m}(0)\right|^{2}\right)^{1 / 2}}, \quad \widetilde{F}_{m}=\frac{F_{m}}{\left(f_{J_{k_{m}}}\left|F_{m}-F_{m}(0)\right|^{2}\right)^{1 / 2}}
$$

and the corresponding source $\widetilde{Q}_{m}=\left(\widetilde{a}_{m}, \widetilde{b}_{m}, \widetilde{c}_{m}, \widetilde{\mu}_{m}\right)$, given by

$$
\widetilde{Q}_{m}=\frac{Q_{m}}{\left(f_{J_{k_{m}}}\left|F_{m}-F_{m}(0)\right|^{2}\right)^{1 / 2}}
$$

Then, by (5.23),

$$
\left\|\widetilde{Q}_{m}\right\|_{X}=o\left(\left|z_{m}\right|\right) \text { as } m \rightarrow \infty .
$$

We shall analyze first the case where $\left|z_{m}\right| \rightarrow \infty$. It will be enough to consider the situation where (6.15) holds. Clearly

$$
f_{J_{k_{m}}}\left|\widetilde{F}_{m}-\widetilde{F}_{m}(0)\right|^{2}=1, \quad f_{J_{k}}\left|\widetilde{F}_{m}-\widetilde{F}_{m}(0)\right|^{2} \leq C \text { for all } k,
$$




$$
\left(f_{J_{k}}\left|\partial_{x}^{7 / 2}\left(\widetilde{F}_{m}-\widetilde{F}_{m}(0)\right)\right|^{2}\right)^{1 / 2} \leq C\left|z_{m}\right| \text { for all } k
$$

and, by (5.15), (5.16),

$$
\begin{gathered}
f_{S_{k}^{(1)}}\left|\nabla^{2} \widetilde{W}_{m}\right|^{2} \rightarrow 0 \text { for any } k, \text { as } m \rightarrow \infty, \\
\left(f_{S_{k}^{(1)}}\left|\nabla^{4} \widetilde{W}_{m}\right|^{2}\right)^{1 / 2}=o\left(\left|z_{m}\right|\right) \text { for any } k, \text { as } m \rightarrow \infty .
\end{gathered}
$$

By (5.12), also

$$
\|\widetilde{W}\|_{Y_{2}} \rightarrow 0
$$

The last relation implies, by (5.7), that

$$
\left(f_{Q_{k n}}\left|\nabla \widetilde{W}_{m}\right|^{2}\right)^{1 / 2}=|\log (1-y)| \cdot o(1)
$$

for $\frac{1}{2}<y<\frac{3}{4}$, where $1-y$ is the distance from $Q_{k n}$ to the line $\{y=1\}$ and $o(1) \rightarrow 0$ if $m \rightarrow \infty$. (The $Q_{k n}$ form partitions of the $L_{j}^{0}$.)

By (7.8) and Poincaré's inequality it then follows that

$$
f_{S_{k}^{(1)}}\left|\nabla \widetilde{W}_{m}\right|^{2} \rightarrow 0 \text { for any } k, \text { if } m \rightarrow \infty,
$$

and, by the trace theorem,

$$
\left(f_{J_{k}}\left|\partial_{x} \widetilde{W}_{m}\right|^{2}\right)^{1 / 2} \rightarrow 0 \text { if } m \rightarrow \infty .
$$

Applying again (7.8), in conjunction with (7.9) and interpolation, we deduce, by the trace imbedding, that

$$
\left(f_{J_{k}}\left|\partial_{x}^{j} \widetilde{W}_{m}\right|^{2}\right)^{1 / 2}=o\left(\left|z_{m}\right|\right) \quad(j=0,1,2,3) .
$$

Using (7.11), (7.12) in the boundary condition $(2.5)$ (for $\widetilde{W}_{m}, \widetilde{F}_{m}$ ), we find that

$$
\left(f_{J_{k}}\left|\partial_{x}^{3} F_{m}\right|^{2}\right)^{1 / 2}=o\left(\left|z_{m}\right|\right) \text {. }
$$


Since further $\left|\widetilde{F}_{m}(0)\right|=o\left(\left|z_{m}\right|^{1 / 2}\right)=o\left(\left|z_{m}\right|\right)$ by (5.16), we also have (by using (7.6))

$$
\left(f_{J_{k}}\left|\widetilde{F}_{m}\right|^{2}\right)^{1 / 2}=o\left(\left|z_{m}\right|\right)
$$

and, consequently,

$$
\left(f_{J_{k}}\left|\partial_{x}^{j} \widetilde{F}_{m}\right|^{2}\right)^{1 / 2}=o\left(\left|z_{m}\right|\right) \quad(1 \leq j \leq 3) .
$$

From (7.12),(7.13) it follows that the functions $\hat{a}_{m}$ (in (6.25)) satisfy

$$
\left(f_{J_{k}}\left|\partial_{x}^{j} \hat{a}_{m}\right|^{2}\right)^{1 / 2}=o\left(\left|z_{m}\right|\right) \text { for } j=0 \text { and } j=\frac{5}{2} \text {. }
$$

Using this relation and (7.13), (7.12) in the boundary condition (2.4), we get

$$
\left(f_{J_{k}}\left|\partial_{x}^{j} \widetilde{F}_{m}\right|^{2}\right)^{1 / 2}=o(1) \quad \text { as } m \rightarrow \infty
$$

for $j=0,1,2$. The proof shows that this last estimate actually holds uniformly with respect to $k$, so that

$$
\sup _{k}\left(f_{J_{k}}\left|\partial_{x}^{j} \widetilde{F}_{m}\right|^{2}\right)^{1 / 2} \rightarrow 0 \text { if } m \rightarrow \infty .
$$

Taking $J_{k}$ with $R_{k} \rightarrow 0$, we deduce that

$$
\widetilde{F}_{m}(0) \rightarrow 0
$$

and therefore

$$
f_{J_{k_{m}}}\left|\widetilde{F}_{m}-\widetilde{F}_{m}(0)\right|^{2} \rightarrow 0 \text { if } m \rightarrow \infty,
$$

a contradiction to the first part of (7.6).

In case $\lim _{m \rightarrow \infty} z_{m}$ is a finite number the proof is the same with $o\left(\left|z_{m}\right|\right)$ replaced by $o(1)$ everywhere.

Suppose next that (7.2) is satisfied. We introduce the functions

$$
\widetilde{W}_{m}=\frac{\left|z_{m}\right| W_{m}}{\left(f_{J_{k_{m}}}\left|\partial_{x}^{7 / 2}\left(F_{m}-F_{m}(0)\right)\right|^{2}\right)^{1 / 2}}, \quad \widetilde{F}_{m}=\frac{\left|z_{m}\right| F_{m}}{\left(f_{J_{k_{m}}}\left|\partial_{x}^{7 / 2}\left(F_{m}-F_{m}(0)\right)\right|^{2}\right)^{1 / 2}}
$$

and proceed to obtain estimates similar to the previous case. Since (7.11) and (7.12) hold not only for $\partial_{x} \widetilde{W}_{m}$ and $\partial_{x}^{3} \widetilde{W}_{m}$ but also for $\partial_{x}^{3 / 2} \widetilde{W}_{m}$ and $\partial_{x}^{7 / 2} \widetilde{W}_{m}$ respectively 
(with the same proof; in deriving the estimate for $\partial_{x}^{7 / 2} \widetilde{W}_{m}$ we use also (7.7)), we obtain from the boundary condition $(2.5)$ for $\left(\widetilde{W}_{m}, \widetilde{F}_{m}\right)$

$$
\left(f_{J_{k_{m}}}\left|\partial^{7 / 2}\left(\widetilde{F}_{m}-\widetilde{F}_{m}(0)\right)\right|^{2}\right)^{1 / 2}=o\left(\left|z_{m}\right|\right)
$$

which is a contradiction.

In Section 8 we show that the case (5.14) can be reduced to the case (5.13) with $R_{k_{m}} \rightarrow 0$.

\section{The CASE $R_{k_{m}} \rightarrow 0$ With $(5.13)$}

If $R_{k_{m}} \rightarrow 0$ then $S_{k_{m}} \rightarrow 0$, and it will be convenient to work with the scaled functions

$$
\begin{gathered}
\hat{W}_{m}(x, y) \equiv \hat{W}_{m}\left(x, y, z_{m}\right)=W_{m}\left(R_{k_{m}} x, R_{k_{m}} y, z_{m}\right), \hat{F}_{m}(x)=F_{m}\left(R_{k_{m}} x\right), \\
\hat{a}_{m}(x)=a_{m}\left(R_{k_{m}} x\right), \hat{b}_{m}(x)=b_{m}\left(R_{k_{m}} x\right), \hat{c}_{m}(x)=c_{m}\left(R_{k_{m}} x\right) .
\end{gathered}
$$

Thus if (5.13) holds then the "sup" in (5.13) is achieved by

$$
g\left(R_{k_{m}}, z_{m}\right)\left[R_{k_{m}}^{2}\left(\left|z_{m}\right|+\frac{1}{R_{k_{m}}^{2}}\right)\left(f_{B}\left|\nabla^{2} \hat{W}_{m}\right|^{2}\right)^{1 / 2}+\left(f_{B}\left|\nabla^{4} \hat{W}_{m}\right|^{2}\right)^{1 / 2}\right],
$$

whereas if (5.15), (5.16) holds then the "sup" in (5.15) is achieved by

$$
\begin{array}{r}
g\left(R_{k_{m}}, z_{m}\right)\left[\left(f_{J} \mid\left(\left|\hat{F}_{m}-\hat{F}_{m}(0)\right|^{2}\right)^{1 / 2} R_{k_{m}}\left(\left|z_{m}\right|+\frac{1}{R_{k_{m}}}\right)\right.\right. \\
\left.+\left(f_{J}\left|\partial_{x}^{7 / 2}\left(\hat{F}_{m}-\hat{F}_{m}(0)\right)\right|^{2}\right)^{1 / 2}\right] .
\end{array}
$$

Here $B$ is a scaled version of $S_{k_{m}}$ of dimensions $\approx 1$ and $J=\bar{B} \cap\{y=0\} ; B$ and $J$ depend on $k_{m}$ but, to keep the notation simple, we shall not indicate this explicitly. In this section we shall consider the case (5.13) and, very briefly (in the paragraph following (8.29)), the case (5.14).

It will be convenient to consider separately the three cases:

$$
\begin{aligned}
& R_{k_{m}} z_{m} \rightarrow \omega, \omega \quad \text { finite and } \quad \neq 0, \\
& R_{k_{m}} z_{m} \rightarrow 0 \\
& R_{k_{m}}\left|z_{m}\right| \rightarrow \infty
\end{aligned}
$$

We shall first consider these cases under the assumption that (6.1) holds. 
Case (8.4). We normalize $\hat{W}_{m}, \hat{F}_{m}$ by

$$
\widetilde{W}_{m}=\frac{\hat{W}_{m}}{\left(f_{B}\left|\nabla^{4} \hat{W}_{m}\right|^{2}\right)^{1 / 2}}, \quad \widetilde{F}_{m}=\frac{\hat{F}_{m}}{\left(f_{B}\left|\nabla^{4} \hat{W}_{m}\right|^{2}\right)^{1 / 2}}
$$

so that

$$
f_{B}\left|\nabla^{4} \widetilde{W}_{m}\right|^{2}=1 .
$$

The functions $\widetilde{W}_{m}, \widetilde{F}_{m}$ satisfy

$$
R_{k_{m}}^{2} z_{m} \Delta \widetilde{W}_{m}-\Delta^{2} \widetilde{W}_{m}=\widetilde{\mu}_{m} \text { in } D_{m} \equiv R_{k_{m}} D
$$

with the boundary conditions

$$
\begin{gathered}
3 \frac{\partial^{3} \widetilde{W}_{m}}{\partial x^{2} \partial y}+\frac{\partial^{3} \widetilde{W}_{m}}{\partial y^{3}}+\sigma \frac{\partial^{3} \widetilde{F}_{m}}{\partial x^{3}}-R_{k_{m}}^{2} z_{m} \frac{\partial \widetilde{W}_{m}}{\partial y}=R_{k_{m}}^{3} \hat{b}_{m} \equiv \widetilde{b}_{m} \quad \text { if } \quad y=0, x<0, \\
\frac{\partial^{2} \widetilde{W}_{m}}{\partial x^{2}}-\frac{\partial^{2} \widetilde{W}_{m}}{\partial y^{2}}=R_{k_{m}}^{2} \hat{c}_{m} \equiv \widetilde{c}_{m} \quad \text { if } \quad y=0, x<0 .
\end{gathered}
$$

Here

$$
\widetilde{\mu}_{m}(x, y)=R_{k_{m}}^{4} \mu_{m}\left(R_{k_{m}} x, R_{k_{m}} y\right) /\left(f_{S_{k_{m}}^{(1)}}\left|\nabla^{4} \widetilde{W}_{m}\right|^{2}\right)^{1 / 2}
$$

From the definition of $k_{m}$ we have

$$
g\left(R_{k}, z_{m}\right) R_{k}^{4}\left(f_{S_{k}}\left|\nabla^{4} W_{m}\right|^{2}\right)^{1 / 2} \leq C g\left(R_{k_{m}}, z_{m}\right) R_{k_{m}}^{4}\left(f_{S_{k_{m}}^{(1)}}\left|\nabla^{4} W_{m}\right|^{2}\right)^{1 / 2}
$$

for any $S_{k}$. Taking $R_{k} \sim R R_{k_{m}}$, we get

$$
R^{4}\left(f_{\left(B_{R} \backslash R_{R / 2}\right)^{+}}\left|\nabla^{4} \widetilde{W}_{m}\right|^{2}\right)^{1 / 2} \leq C \frac{g\left(R_{k_{m}}, z_{m}\right)}{g\left(R R_{k_{m}}, z_{m}\right)} \leq C h(R)
$$

if $m$ is large enough, where, by (5.1),

$$
h(R)= \begin{cases}R^{\frac{1}{2}+\epsilon} & \text { if } R>1, \\ R^{\frac{3}{2}-\epsilon} & \text { if } R<1 .\end{cases}
$$


Similarly, using (6.1) we deduce that

$$
R^{2}\left(f_{\left(B_{R} \backslash B_{R / 2}\right)^{+}}\left|\nabla^{2} \widetilde{W}_{m}\right|^{2}\right)^{1 / 2} \leq C h(R) .
$$

Since $\widetilde{W}_{m}(x, 0)=\partial_{y} \widetilde{W}_{m}(x, 0)=0$ if $x>0$, from the last estimate and Poincaré's inequality we deduce that

$$
\left|\widetilde{W}_{m}\right|_{L^{\infty}\left(\left(B_{R} \backslash R_{R / 2}\right)^{+}\right)} \leq C h(R) .
$$

We next recall that, by (5.13),

$$
\begin{gathered}
g\left(R_{k}, z_{m}\right)\left[R_{k}\left(\left|z_{m}\right|+\frac{1}{R_{k}}\right)\left(f_{J_{k}}\left|F_{m}-F_{m}(0)\right|^{2}\right)^{1 / 2}\right. \\
\left.+R_{k}^{7 / 2}\left(f_{J_{k}}\left|\partial_{x}^{7 / 2}\left(F_{m}-F_{m}(0)\right)\right|^{2}\right)^{1 / 2}\right] \\
\leq C g\left(R_{k_{m}}, z_{m}\right) R_{k_{m}}^{4}\left(f_{S_{k_{m}}^{(1)}}\left|\nabla^{4} W_{m}\right|^{2}\right)^{1 / 2}
\end{gathered}
$$

for all $k$. Taking $R_{k} \sim R R_{k_{m}}$, we deduce that

$$
\left(f_{J_{R}}\left|\widetilde{F}_{m}-\widetilde{F}_{m}(0)\right|^{2}\right)^{1 / 2}+R^{7 / 2}\left(f_{J_{R}}\left|\partial_{x}^{7 / 2}\left(\widetilde{F}_{m}-\widetilde{F}_{m}(0)\right)\right|^{2}\right)^{1 / 2} \leq C h(R),
$$

where $J_{R}=\left(B_{R} \backslash B_{R / 2}\right) \cap\{y=0\}$. We also have

$$
\begin{aligned}
\left|\widetilde{F}_{m}(0)\right| & \leq \frac{1}{\left|z_{m}\right|^{1 / 2}} g\left(R_{k_{m}}, z_{m}\right)\left(f_{B}\left|\nabla^{4} \widetilde{W}_{m}\right|^{2}\right)^{1 / 2} \\
& \leq \frac{1}{\left|z_{m}\right|^{1 / 2}}\left(\frac{1}{\left|z_{m}\right|^{1 / 2}}\right)^{2 \epsilon} R_{k_{m}}^{-\frac{1}{2}-\epsilon} \leq C
\end{aligned}
$$

and therefore, by (8.20),

$$
\widetilde{F}_{m} \rightarrow f \text { in } C^{0}[-N, 0] \text { for any } N>0,
$$

for some function $f$. (More precisely, we have to go to a subsequence of $\widetilde{F}_{m}$.)

From the definition of $\widetilde{\mu}_{m}$ and (5.11) we get, for any $R>0$,

$$
\left(f_{\left(B_{R} \backslash B_{R / 2}\right)^{+}}\left|\widetilde{\mu}_{m}\right|^{2}\right)^{1 / 2} \rightarrow 0 \text { as } m \rightarrow \infty \text {. }
$$


Similarly, we deduce that

$$
\widetilde{a}_{m} \rightarrow 0, \widetilde{b}_{m} \rightarrow 0, \widetilde{c}_{m} \rightarrow 0 \text { in } L_{\mathrm{loc}}^{2}(-\infty, 0) .
$$

From (8.18), (8.15) and (8.21)-(8.23) we easily conclude that

$$
\widetilde{W}_{m} \rightarrow \varphi \text { weakly in } H_{\text {loc }}^{4}(D \backslash\{0\}),
$$

and $\varphi, f$ form a solution to the elliptic problem

$$
\begin{aligned}
& \Delta^{2} \varphi=0 \text { in } \mathbb{R}_{+}^{2}, \\
& \varphi(x, 0)=\partial_{y} \varphi(x, 0)=0 \quad \text { if } \quad x>0 \text {, } \\
& \omega f+\beta_{0} \frac{\partial f}{\partial x}-\frac{\partial \varphi}{\partial x}=0 \quad \text { if } \quad y=0, x<0, \\
& 3 \frac{\partial^{3} \varphi}{\partial x^{2} \partial y}+\frac{\partial^{3} \varphi}{\partial y^{3}}+\sigma \frac{\partial^{3} f}{\partial x^{3}}=0 \quad \text { if } \quad y=0, x<0, \\
& \frac{\partial^{2} \varphi}{\partial x^{2}}-\frac{\partial^{2} \varphi}{\partial y^{2}}=0 \quad \text { if } \quad y=0, x<0
\end{aligned}
$$

and

$$
|\varphi(x, y)| \leq C h(r), \quad|f(x)| \leq C(1+|x|)^{\frac{1}{2}+\epsilon}
$$

where $r=\sqrt{x^{2}+y^{2}}$. By a Liouville type theorem (Theorem 10.2 of [7]) the only solution of (8.24)-(8.29) is $\varphi \equiv 0, f \equiv 0$. Consequently

$$
\widetilde{W}_{m} \rightarrow 0 \text { uniformly in compact subsets of } D,
$$

and weakly in $H_{\text {loc }}^{4}(D \backslash\{0,0\})$. Using this fact we can now use truncation, as in $\S 6$, and Lemma 4.3 in order to prove that

$$
\left(f_{B}\left|\nabla^{4} \widetilde{W}_{m}\right|^{2}\right)^{1 / 2} \rightarrow 0 \text { as } m \rightarrow \infty,
$$

which is a contradiction to (8.8).

We now digress in order to analyze the case where (5.14) holds.

We define $R_{k_{m}}=1 /\left|z_{m}\right|$ and proceed to normalize by

$$
\widetilde{W}_{m}=\frac{\hat{W}_{m}}{\hat{F}_{m}(0)}, \quad \widetilde{F}_{m}=\frac{\hat{F}_{m}}{\hat{F}_{m}(0)} .
$$

Since $\widetilde{F}_{m}(0)=1$ and $\left|z_{m}\right|^{1 / 2}=R_{k_{m}}^{-1 / 2}=g\left(R_{k_{m}}, z_{m}\right)$, it follows that (8.17), (8.18) and (8.20) hold with the same function $h(R)$ as in (8.16)'. Consequently, (8.21) holds, $\widetilde{W}_{m} \rightarrow \varphi$, and $(\varphi, f)$ forms a solution to (8.24)-(8.29). By $[7$, Theorem $10.2] \varphi \equiv 0, f \equiv 0$. But since $\widetilde{F}_{m}(0)=1$, we have $f \not \equiv 0$ (by $(8.21)$, which is a contradiction.

We next consider:

Case (8.5). We can proceed precisely as before, except for one difficulty, namely, we cannot assert that $\widetilde{F}_{m}(0)$ is bounded since $R_{k_{m}} z_{m} \rightarrow 0$. To overcome this difficulty we introduce the differences

$$
\widetilde{G}_{m}=\widetilde{F}_{m}-\widetilde{F}_{m}(0)
$$


and rewrite (8.11) in the form

$$
R_{k_{m}} z_{m} \widetilde{F}_{m}(0)+R_{k_{m}} z_{m} \widetilde{G}_{m}+\beta_{0} \frac{\partial \widetilde{G}_{m}}{\partial x}-\frac{\partial \widetilde{W}_{m}}{\partial x}=\widetilde{a}_{m} .
$$

Then, for any $R>0,(8.17),(8.18)$ and (8.20) hold with

$$
h(r)=r^{\frac{3}{2}-\epsilon}
$$

provided $m$ is large enough, and

$$
\widetilde{W}_{m} \rightarrow \varphi, \quad \widetilde{G}_{m} \rightarrow f
$$

in suitable norms. Consequently, (8.30) yields

$$
R_{k_{m}} z_{m} \widetilde{F}_{m}(0) \rightarrow c^{*}
$$

If $c^{*}=0$ then we can use a Liouville type theorem (Theorem 10.1 of [7]) to deduce that $\varphi \equiv 0$ and $f \equiv 0$, and we can proceed to derive a contradiction as in case (8.4). It therefore remains to consider the case

$$
c^{*} \neq 0 \text {. }
$$

Set $g=f+c^{*} x / \beta_{0}$. Then by the Liouville type theorem cited above, applied to $(\varphi, g)$, we have

$$
\varphi \equiv 0, \quad g \equiv 0 .
$$

Consequently

$$
f(x)=-\frac{c^{*}}{\beta_{0}} x
$$

and

$$
\int_{-N}^{0}\left|\widetilde{F}_{m}-\widetilde{F}_{m}(0)+\frac{c^{*}}{\beta_{0}} x\right|^{2} d x \rightarrow 0 \text { if } m \rightarrow \infty, \quad \text { for any } N>0 .
$$

On the other hand, by $(8.20)$, if $0<N<1$ then

$$
\begin{aligned}
\int_{-N}^{0}\left|\widetilde{F}_{m}-\widetilde{F}_{m}(0)\right|^{2} & \leq C \sum_{0<R_{i}<N} R_{i} f_{J_{R_{i}}}\left|\widetilde{F}_{m}-\widetilde{F}_{m}(0)\right|^{2} \\
& \leq C \sum_{0<R_{i}<N} R_{i} h^{2}\left(R_{i}\right) \leq C \sum_{0<R_{i}<N} R_{i}^{4-2 \epsilon} \leq C N^{4-2 \epsilon},
\end{aligned}
$$

since we can take $R_{i} \approx N / 2^{i}$. A comparison with (8.33) shows that $c^{*}=0$, a contradiction.

It remains to consider:

Case (8.6). We divide it into three subcases:

$$
\begin{aligned}
& \text { (a) } R_{k_{m}}^{2}\left|z_{m}\right| \rightarrow \infty, \\
& \text { (b) } R_{k_{m}}^{2} z_{m} \rightarrow \omega, \quad \omega \text { finite and } \neq 0, \\
& \text { (c) } \left.R_{k_{m}}^{2} z_{m} \rightarrow 0 \text { (but } R_{k_{m}}\left|z_{m}\right| \rightarrow \infty\right) .
\end{aligned}
$$

Case (a). The situation is similar to that in $\S 6$ with $\left|z_{m}\right| \rightarrow \infty$. Since $R_{k_{m}} \gg$ $\left|z_{m}\right|^{-1 / 2}$ we need to use, on $S_{k}^{(2)}$, the estimate (6.14) for $\widetilde{W}_{m}$ and similar estimates 
for $\nabla^{2}\left(z_{m} \widetilde{W}_{m}\right)$ (cf. (6.17)) and $\nabla^{4} \widetilde{W}_{m}$. On $S_{k}^{(1)}$ the estimates remain as before (for example, (8.14) holds with $S_{k}=S_{k}^{(1)}$ and (8.20) holds with $h(R)=R^{(1 / 2)-\epsilon}$ ). If we set $\varphi=\lim _{m \rightarrow \infty}\left(z_{m} \widetilde{W}_{m}\right)$ then $\varphi$ satisfies

$$
\begin{aligned}
\Delta \varphi=0 \quad \text { in } \quad \mathbb{R}_{+}^{2}, \\
\partial_{y} \varphi(x, 0)=0 \quad \text { if } \quad-\infty<x<0, \\
\varphi(x, 0)=0 \quad \text { if } \quad 0<x<\infty,
\end{aligned}
$$

and

$$
|\varphi| \leq C_{\epsilon_{0}} y|\log y| \text { if } x>\epsilon_{0}, 0<y<1 \quad\left(\text { for any } \epsilon_{0}>0\right)
$$

whereas

$$
|\varphi| \leq C r^{\frac{1}{2}-\epsilon} \text { if } r>1
$$

Near $r=0$ we also obtain the bound

$$
|\varphi| \leq C r^{-\frac{3}{2}-\epsilon}
$$

for $x<0$. From the series expansion of $\varphi$ near the origin we then infer that

$$
\varphi=A r^{-3 / 2} \sin \frac{3 \theta}{2}+B r^{-1 / 2} \sin \frac{\theta}{2}+O\left(r^{1 / 2}\right)
$$

as $r \rightarrow 0$. The function

$$
\psi \equiv \varphi-A r^{-3 / 2} \sin \frac{3 \theta}{2}-B r^{-1 / 2} \sin \frac{\theta}{2}
$$

is harmonic in $\mathbb{R}_{+}^{2}$, satisfies the same boundary and growth conditions (8.35)-(8.37) as $\varphi$, and is continuous at the origin. By comparison, for any small $\delta>0$

$$
\pm \psi<\delta r^{1 / 2} \sin \frac{\theta}{2} \text { in } \mathbb{R}_{+}^{2} \cap\left\{x^{2}+y^{2}<\rho^{2}\right\}
$$

if $\rho$ is large enough so that the inequality holds at $r=\rho$. It follows that $\psi \equiv 0$, and thus

$$
\varphi=A r^{-3 / 2} \cos \frac{3 \theta}{2}+B r^{-1 / 2} \cos \frac{\theta}{2} .
$$

Lemma 8.1. $A=B=0$, so that $\varphi \equiv 0$.

The proof, which is similar to that of Lemma 6.1, is given in $\S 15$.

Since $\varphi \equiv 0$,

$$
\widetilde{W}_{m} \rightarrow 0 \text { uniformly in compact subsets of } \overline{\mathbb{R}_{+}^{2}} \backslash\{(0,0)\} .
$$

We can now use cutoff, interpolation and Lemma 4.3 (as in §6) to deduce that

$$
\left(f_{B}\left|\nabla^{4} \widetilde{W}_{m}\right|^{2}\right)^{1 / 2} \rightarrow 0 \text { as } m \rightarrow \infty
$$

which is a contradiction.

Case (b). In this case $\varphi=\lim _{m \rightarrow \infty} \widetilde{W}_{m}$ satisfies

$$
\omega \Delta \varphi-\Delta^{2} \varphi=0
$$


in $\mathbb{R}_{+}^{2}$. Multiplying (8.11) by a test function and integrating, and using the assumption $R_{k_{m}}\left|z_{m}\right| \rightarrow \infty$, we deduce that $\widetilde{F}_{m} \rightarrow 0$ in the distribution sense. Consequently the left-hand side of (8.12) converges, in a weak sense, to

$$
3 \frac{\partial^{3} \varphi}{\partial x^{2} \partial y}+\frac{\partial^{3} \varphi}{\partial y^{3}}-\omega \frac{\partial \varphi}{\partial y}
$$

The right-hand sides of (8.12) and (8.13) converge weakly to zero since $\left\|\widetilde{Q}_{m}\right\|_{X} \rightarrow 0$. Using elliptic estimates ([1], [2] and [9]) we find that $\varphi$ is a classical solution to the elliptic problem

$$
\begin{array}{rll}
\omega \Delta \varphi=\Delta^{2} \varphi & \text { in } & \mathbb{R}_{+}^{2}, \\
\varphi(x, 0)=\partial_{y} \varphi(x, 0)=0 & \text { if } & x>0, \\
3 \frac{\partial^{3} \varphi}{\partial x^{2} \partial y}+\frac{\partial^{3} \varphi}{\partial y^{3}}-\omega \frac{\partial \varphi}{\partial y}=0 & \text { if } & y=0, x<0, \\
\frac{\partial^{2} \varphi}{\partial x^{2}}-\frac{\partial^{2} \varphi}{\partial y^{2}}=0 & \text { if } & y=0, x<0,
\end{array}
$$

and, furthermore, from the definition of $g(R, z)$ and the analysis that let to (8.29),

$$
|\varphi(x, y)| \leq\left\{\begin{array}{l}
r^{\frac{1}{2}-\epsilon} \text { if } r>1 \\
r^{\frac{1}{2}+\epsilon} \text { if } r<1 .
\end{array}\right.
$$

We appeal to another Liouville type theorem (Theorem 1.1 of [7]) to conclude that $\varphi \equiv 0$. Hence (8.39) holds and we can proceed as before to derive (8.40), which is a contradiction.

Case (c). This case is treated similarly to case (b), the only difference being that $\omega=0$ and

$$
|\varphi(x, y)| \leq C r^{\frac{1}{2}+\epsilon} \text { in } \mathbb{R}_{+}^{2} .
$$

Here we need to appeal to a different Liouville type theorem (Theorem 10.1 of [7]).

So far we have dealt with the case (6.1). If (6.2) holds then we normalize $\hat{W}_{m}$, $\hat{F}_{m}$ by dividing by

$$
\left|z_{m}\right|\left(f_{S_{k_{m}}^{(1)}}\left|\nabla^{2} \hat{W}_{m}\right|^{2}\right)^{1 / 2}
$$

and proceed as before with minor changes (cf. $\S 6$ ).

9. The CASe $R_{k_{m}} \rightarrow 0$ With (5.15), (5.16)

We again treat separately the three cases (8.4), (8.5) and (8.6). 
Case (8.4). We normalize by

$$
\begin{aligned}
\widetilde{W}_{m} & =\frac{\hat{W}_{m}}{\left(f_{J}\left|\hat{F}_{m}-\hat{F}_{m}(0)\right|^{2}\right)^{1 / 2}+\left(f_{J}\left|\partial_{x}^{7 / 2}\left(\hat{F}_{m}-\hat{F}_{m}(0)\right)\right|^{2}\right)^{1 / 2}}, \\
\widetilde{F}_{m} & =\frac{\hat{F}_{m}}{\left(f_{J}\left|\hat{F}_{m}-\hat{F}_{m}(0)\right|^{2}\right)^{1 / 2}+\left(f_{J}\left|\partial_{x}^{7 / 2}\left(\hat{F}_{m}-\hat{F}_{m}(0)\right)\right|^{2}\right)^{1 / 2}} .
\end{aligned}
$$

Then

$$
\left(f_{J}\left|\widetilde{F}_{m}-\widetilde{F}_{m}(0)\right|^{2}\right)^{1 / 2}+\left(f_{J}\left|\partial_{x}^{7 / 2}\left(\widetilde{F}_{m}-\widetilde{F}_{m}(0)\right)\right|^{2}\right)^{1 / 2}=1 .
$$

Also, since $g\left(R_{k_{m}}, z_{m}\right) \sim R_{k_{m}}^{-1 / 2}$,

$$
\left|\widetilde{F}_{m}(0)\right| \ll \frac{C}{\left|z_{m}\right|^{1 / 2} R_{k_{m}}^{1 / 2}} \leq C \quad \text { if } m \rightarrow \infty .
$$

We can proceed to analyze $\widetilde{W}_{m}, \widetilde{F}_{m}$ as before, proving that $\widetilde{W}_{m} \rightarrow 0$ weakly in $H^{4}$ and $\widetilde{F}_{m} \rightarrow 0$ in some norm, weaker than $H^{7 / 2}$. Next truncate $\widetilde{W}_{m}, \widetilde{F}_{m}$ as in $\S 6$, obtain an elliptic system for the truncated pair, and apply Lemma 4.3 to deduce the stronger convergence:

$$
\left(f_{J}\left|\widetilde{F}_{m}-\widetilde{F}_{m}(0)\right|^{2}\right)^{1 / 2}+\left(f_{J}\left|\partial_{x}^{7 / 2}\left(\widetilde{F}_{m}-\widetilde{F}_{m}(0)\right)\right|^{2}\right)^{1 / 2} \rightarrow 0
$$

this is a contradiction to (9.2).

Case (8.5). We again normalize as in (9.1) and proceed precisely as in case (8.5) in $\S 8$ to prove that

$$
\left.\widetilde{F}_{m}-\widetilde{F}_{m}(0) \rightarrow-\frac{c^{*}}{\beta_{0}} x \quad \text { in } \quad L^{2}(-N, 0)\right), \quad \text { for any } N>0 .
$$

Let $\epsilon_{0}$ be any small positive number. For each $m$ let $\rho_{m}$ be the largest $R$ such that $R R_{k_{m}}\left|z_{m}\right| \leq 1$ and, for $R \leq \rho_{m}$,

$$
\left(f_{J_{R}}\left|\widetilde{F}_{m}-\widetilde{F}_{m}(0)\right|^{2}\right)^{1 / 2}+R^{7 / 2}\left(f_{J_{R}}\left|\partial_{x}^{7 / 2}\left(\widetilde{F}_{m}-\widetilde{F}_{m}(0)\right)\right|^{2}\right)^{1 / 2} \geq \epsilon_{0} R ;
$$

clearly $\rho_{m}>1$ if $m$ is large. If $\rho_{m} R_{m} z_{m} \rightarrow \omega \neq 0$ then we can proceed as in case (8.4) (replacing $R_{k_{m}}$ by $\rho_{m} R_{k_{m}}$ in (8.1)) and prove that the left-hand side of (9.4) is convergent to zero, which is a contradiction. Thus it remains to consider the case

$$
\rho_{m} R_{k_{m}} z_{m} \rightarrow 0 \text {. }
$$


We define

$$
F_{m}^{*}=\frac{\widetilde{F}_{m}}{\rho_{m}}
$$

Then (9.5) becomes

$$
\left(f_{J_{R}}\left|F_{m}^{*}-F_{m}^{*}(0)\right|^{2}\right)^{1 / 2}+\left(f_{J_{R}}\left|\partial_{x}^{7 / 2}\left(F_{m}^{*}-F_{m}^{*}(0)\right)\right|^{2}\right)^{1 / 2} \geq \epsilon_{0} R / \rho_{m},
$$

and we proceed as before up to the asssertion (8.31), which is now

$$
\rho_{m} R_{k_{m}} z_{m} F_{m}^{*}(0) \rightarrow c^{*} .
$$

This implies that $R_{k_{m}} z_{m} \widetilde{F}_{m}(0) \rightarrow c^{*}$, and therefore $c^{*}$ is independent of $\epsilon_{0}$. Further, as before,

$$
G_{m}^{*}=F_{m}^{*}-F_{m}^{*}(0) \rightarrow-\frac{c^{*}}{\beta_{0}} x \text { in } L^{2}(-N, 0)
$$

for any $N>0$. Because of (9.6), equality must hold in (9.7) for $R=\rho_{m}$ (otherwise $\rho_{m}$ could be increased, which is a contradiction to the definition of $\rho_{m}$ ), and so

$$
\left(f_{J_{\rho_{m}}}\left|F_{m}^{*}-F_{m}^{*}(0)\right|^{2}\right)^{1 / 2} \leq \epsilon_{0}
$$

Taking $m \rightarrow \infty$ and comparing with (9.4) we see that $\left|c^{*}\right| \leq C \epsilon_{0}$, where $C$ is a constant independent of $\epsilon_{0}$. Since $\epsilon_{0}$ can be taken arbitrarily small, $c^{*}$ must vanish, and then, as in $\S 8$, we apply a Liouville type theorem (Theorem 10.1 of [7]) to deduce that $\varphi=0, f=0$ and proceed as before to derive a contradiction.

Case (8.6). We divide it into subcases (a), (b), (c), as in $\S 8$, and normalize by

$$
\widetilde{W}_{m}=\frac{\hat{W}_{m}}{A_{m}}, \hat{F}_{m}=\frac{\hat{F}_{m}}{A_{m}}
$$

where

$$
\begin{aligned}
A_{m}=\left[R_{k_{m}}\left(\left|z_{m}\right|+\frac{1}{R_{k_{m}}}\right)\right. & \left(f_{J}\left|\hat{F}_{m}-\hat{F}_{m}(0)\right|^{2}\right)^{1 / 2} \\
& \left.+\left(f_{J}\left|\partial_{x}^{7 / 2}\left(\hat{F}_{m}-\hat{F}_{m}(0)\right)\right|^{2}\right)^{1 / 2}\right] .
\end{aligned}
$$

Then

$$
R_{k_{m}}\left(\left|z_{m}\right|+\frac{1}{R_{k_{m}}}\right)\left(f_{J}\left|\widetilde{F}_{m}-\widetilde{F}_{m}(0)\right|^{2}\right)^{1 / 2}+\left(f_{J}\left|\partial_{x}^{7 / 2}\left(\widetilde{F}_{m}-\widetilde{F}_{m}(0)\right)\right|^{2}\right)^{1 / 2}=1 .
$$


Consider first the cases (a), (b). Using (5.16), we can argue as in $\S 7$ to deduce that (7.10) and (7.11) hold (with $S_{k}^{(1)}, J_{k}$ properly scaled). Next, by (5.1) and the assumptions in (a), (b),

$$
g\left(R_{k_{m}}, z_{m}\right) \approx R_{k_{m}}^{-\frac{1}{2}+\epsilon}
$$

and therefore

$$
\left|\widetilde{F}_{m}(0)\right| \leq \frac{C R_{k_{m}}^{\epsilon}}{\left|z_{m}\right|^{1 / 2} R_{k_{m}}^{1 / 2}} \rightarrow 0
$$

It follows that

$$
\left(f_{J_{R}}\left|\widetilde{F}_{m}\right|^{2}\right)^{1 / 2} \leq\left(f_{J_{R}}\left|\widetilde{F}_{m}-\widetilde{F}_{m}(0)\right|^{2}\right)^{1 / 2}+\left|\widetilde{F}_{m}(0)\right| \leq C .
$$

Using (7.11), (9.10) and the assumption $R_{k_{m}}\left|z_{m}\right| \rightarrow \infty$ in the boundary condition (8.11), we deduce that

$$
\widetilde{F}_{m} \rightarrow 0 \text { in } H^{-1}\left(J_{R}\right) \text { for any } R>0 ;
$$

and, consequently, also

$$
\widetilde{F}_{m}-\widetilde{F}_{m}(0) \rightarrow 0 \text { in } H^{-1}
$$

Noting that

$$
f_{J_{R}}\left|\widetilde{F}_{m}-\widetilde{F}_{m}(0)\right|^{2}+f_{J_{R}}\left|\partial_{x}^{7 / 2}\left(\widetilde{F}_{m}-\widetilde{F}_{m}(0)\right)\right|^{2}
$$

is bounded for any $R>0$, we then deduce by interpolation [9, p. 49] that $\widetilde{F}_{m}-$ $\widetilde{F}_{m}(0) \rightarrow 0$ in $L^{2}$. We can now use truncation, interpolation and Lemma 4.3 to deduce that

$$
f_{J}\left|\partial_{x}^{j}\left(\widetilde{F}_{m}-\widetilde{F}_{m}(0)\right)\right|^{2} \rightarrow 0
$$

for $j=7 / 2$, as well as for any smaller $j$. We can next apply truncation and interpolation as in $\S 7$ to deduce that

$$
\left(\left|z_{m}\right|+\frac{1}{R_{k_{m}}}\right) R_{k_{m}}\left(f_{J}\left|\widetilde{F}_{m}-\widetilde{F}_{m}(0)\right|^{2}\right)^{1 / 2} \rightarrow 0 ;
$$

and, together with (9.11), this is a contradiction to the normalization (9.9). Case (c) is treated similarly. The only difference is that instead of (9.10) we have

$$
g\left(R_{k_{m}}, z_{m}\right)=\left|z_{m}\right|^{-\epsilon} R_{k_{m}}^{-\frac{1}{2}-\epsilon}
$$

and

$$
\left|\widetilde{F}_{m}(0)\right| \leq \frac{C}{\left|z_{m}\right|^{1 / 2}\left|z_{m}\right|^{\epsilon} R_{k_{m}}^{\frac{1}{2}+\epsilon}} \leq \frac{C}{\left(R_{k_{m}}\left|z_{m}\right|\right)^{\frac{1}{2}+\epsilon}} .
$$

Since $R_{k_{m}}\left|z_{m}\right| \rightarrow \infty, \widetilde{F}_{m}(0) \rightarrow 0$, and we can proceed as before. 


\section{Completion of the proof of Lemma 5.1}

We have proved so far that if

$$
\left\|\left(W_{m}, F_{m}\right)\right\|_{Y_{1}} \gg\left\|W_{m}\right\|_{Y_{2}}+\left\|W_{m}\right\|_{Y_{3}}
$$

for some $c>0$, then we get a contradiction to (5.11). Suppose next that (5.17) (or (5.18)) holds. Then we can proceed as in the previous sections, making only small changes. If $R_{k_{m}} \rightarrow 0$ then we rescale the $W_{m}, F_{m}$ as in (8.1); we also normalize $\hat{W}_{m}, \hat{F}_{m}$ so that, for the new $\widetilde{W}_{m}, \widetilde{F}_{m}$,

the expression in brackets in (5.17) (or (5.18)) is equal to 1 at $k=k_{m}$.

The arguments in Sections 6 and 8 show that $\widetilde{W}_{m} \rightarrow 0$ if $m \rightarrow \infty$. We can then use cutoff, interpolation and Lemmas 4.1, 4.2 to derive a contradiction to (10.1), by showing that the expression in brackets in (5.17) (or (5.18)) converges to zero if $m \rightarrow \infty$. Here, basically, instead of

$$
\left|z_{m}\right| R^{4}\left(\int_{S_{k}^{(1)}}\left|\nabla^{2} W_{m}\right|^{2}\right)^{1 / 2} \text { and } R^{4}\left(\int_{S_{k}^{(1)}}\left|\nabla^{4} W_{m}\right|^{2}\right)^{1 / 2}
$$

we have to work with

$$
\left|z_{m}\right| R^{3}\left(\int_{S_{k}^{(2)}}\left|y \nabla^{2} W_{m}\right|^{2}\right)^{1 / 2} \text { and } R^{3}\left(\int_{S_{k}^{(2)}}\left|y \nabla^{4} W_{m}\right|^{2}\right)^{1 / 2}
$$

(Actually, $S_{k}^{(2)}$ should be replaced by the $Q_{k n}$ and the factor $y$ in $Q_{k n}$ should be replaced by the $d_{k n}$.) The factor $y$ dimensionally fits with all the interpolations which we need to perform.

\section{Extension of Lemma 5.1}

We shall need an extension of Lemma 5.1 in which we add under the $\max \{\cdots\}$ in (5.6) the expression

$$
\begin{aligned}
\|(W, F)\|_{Z} \equiv \sup _{k}\left[g\left(R_{k}, z\right)[\right. & R_{k}^{3+1 / 2}|z|\left(f_{J_{k}}\left|\partial_{x}^{1 / 2} \partial_{y} W(x, 0)\right|^{2}\right)^{1 / 2} \\
& +R_{k}^{3+1 / 2}|z|^{1+1 / 4}\left(f\left|\partial_{y} W(x, 0)\right|^{2}\right)^{1 / 2} \\
& \left.\left.+R_{k}^{7 / 2}|z|\left(f_{J_{k}}\left|D_{x}^{5 / 2}(F(x)-F(0))\right|^{2}\right)^{1 / 2}\right]\right] .
\end{aligned}
$$


Lemma 11.1. Let $z, W, F$ be as in Lemma 5.1. If the expression in (11.1) is added in the left-hand side of (5.6) under the "max" sign, then the left-hand side of (5.6) still remains bounded by

$$
C|z|^{\frac{\epsilon}{2}+\frac{1}{2}}|\log | z||\|Q\|_{X} .
$$

Proof. It suffices to show that the expression in (11.1) is bounded by the right-hand side of (5.6). Consider the scaled functions

$$
\hat{W}(x, y)=W\left(R_{k} x, R_{k} y\right), \quad \hat{F}(x)=F\left(R_{k} x\right)
$$

and the corresponding scaled $\hat{\mu}, \hat{a}, \hat{b}, \hat{c}$. Then

$$
\begin{aligned}
& \left(R_{k}^{2} z\right) \Delta \hat{W}-\Delta^{2} \hat{W}=R_{k}^{4} \hat{\mu}, \\
& \hat{W}(x, 0)=\partial_{y} \hat{W}(x, 0)=0 \text { if } x>0, \\
& R_{k} z \hat{F}+\beta_{0} \frac{\partial \hat{F}}{\partial x}-\frac{\partial \hat{W}}{\partial x}=R_{k} \hat{a} \text { if } y=0, x<0, \\
& 3 \frac{\partial^{3} \hat{W}}{\partial x^{2} \partial y}+\frac{\partial^{3} \hat{W}}{\partial y^{3}}+\sigma \frac{\partial^{3} \hat{F}}{\partial x^{3}}-\left(R_{k}^{2} z\right) \frac{\partial \hat{W}}{\partial y}=R_{k}^{3} \hat{b} \text { if } y=0, x<0, \\
& \frac{\partial^{2} \hat{W}}{\partial x^{2}}-\frac{\partial^{2} \hat{W}}{\partial y^{2}}=R_{k}^{2} \hat{c} \text { if } y=0, x<0 .
\end{aligned}
$$

Next we introduce a cutoff function $\eta(x, y)$ with $\partial^{j} \eta / \partial y^{i}=0$ at $y=0(0 \leq j \leq$ $3)$, and set

$$
\widetilde{W}=\eta \hat{W}, \widetilde{F}=\eta \hat{F}
$$

Then $\widetilde{W}$ satisfies

$$
\left(R_{k}^{2} z\right) \Delta \widetilde{W}-\Delta^{2} \widetilde{W}=\widetilde{\mu} \text { in } \mathbb{R}_{+}^{2},
$$

where

$$
\widetilde{\mu}=\hat{H}\left(\partial^{3} \hat{W}, \partial^{2} \hat{W}, \partial \hat{W}, \hat{W}, z \partial \hat{W}, z \hat{W}\right)
$$

is a linear combination of the indicated variables, and the boundary conditions (11.4)-(11.7) with $R_{k} \hat{a}, R_{k}^{3} \hat{b}, R_{k}^{2} \hat{c}$ replaced by

$$
\begin{aligned}
& \widetilde{a}=R_{k} \eta \hat{a}+\beta_{0} \hat{F} \partial_{x} \eta-\hat{W} \partial_{x} \eta, \\
& \widetilde{b}=R_{k}^{3} \eta \hat{b}+3 \partial_{y} \hat{W} \cdot \partial_{x}^{2} \eta-6 \frac{\partial \hat{W}}{\partial x \partial y} \partial_{x} \eta-6 \hat{W} \partial_{x}^{3} \eta-3 \sigma \partial_{x} \hat{W} \cdot \partial_{x}^{2} \eta-3 \sigma \frac{\partial^{2} \hat{W}}{\partial x^{2}} \partial_{x} \eta, \\
& \widetilde{c}=R_{k}^{2} \eta \hat{c}-\hat{W} \partial_{x}^{2} \eta-2 \partial_{x} W \cdot \partial_{x} \eta .
\end{aligned}
$$

We now use Lemma 4.3 in order to estimate

$$
\begin{aligned}
& |\widetilde{\epsilon} z|\left(\int_{-\infty}^{0}\left|\nabla^{3 / 2} \widetilde{W}(x, 0)\right|^{2}\right)^{1 / 2}+|\widetilde{\epsilon} z|^{1+1 / 4}\left(\int_{-\infty}^{0}\left|\partial_{y} \widetilde{W}(x, 0)\right|^{2}\right)^{1 / 2} \\
& \quad+|z|\left(\int_{-\infty}^{0}\left|D_{x}^{5 / 2} \widetilde{F}(x)\right|^{2}\right)^{1 / 2} \quad\left(\widetilde{\epsilon}=R_{k}^{2}\right)
\end{aligned}
$$


by (11.2). (Here we have replaced the parameter $\epsilon$ in Lemma 4.3 by $\widetilde{\epsilon}$ in order not to confuse it with the $\epsilon$ that occurs in (11.2).) Actually we get also lower order derivatives of $\hat{W}, \hat{F}$ on the right-hand side (as in $\S 6$ ), but these can be estimated by Lemma 5.1 and interpolation. In particular we note that by writing

$$
D_{x}\left(\hat{F} \partial_{x} \eta\right)=D_{x}\left((\hat{F}-\hat{F}(0)) \partial_{x} \eta\right)+D_{x}\left(\hat{F}(0) \partial_{x} \eta\right)
$$

we can estimate the lower order derivatives of $\hat{F}$; for example, to estimate $D_{x}^{5 / 2} \hat{F}$, we use the fact that both $D_{x}^{5 / 2}(\hat{F}-\hat{F}(0))$ and $\hat{F}(0)$ are bounded by terms in (5.6).

Going back to the original coordinates, the bound (11.2) for the expression (11.9) yields the bound

$$
\begin{gathered}
g\left(R_{k}, z\right)\left[|\widetilde{\epsilon} z| R_{k}^{3 / 2}\left(f_{J_{k}}\left|\nabla^{3 / 2} W(x, 0)\right|^{2}\right)^{1 / 2}+|\widetilde{\epsilon} z|^{1+1 / 4} R_{k}\left(f_{J_{k}}\left|\partial_{y} W(x, 0)\right|^{2}\right)^{1 / 2}\right. \\
\left.+|z| R_{k}^{5 / 2}\left(f_{J_{k}}\left|D_{x}^{7 / 2}(F(x)-F(0))\right|^{2}\right)^{1 / 2}\right] \leq C|z|^{\frac{\epsilon}{2}+\frac{1}{2}}|\log | z||\|Q\|_{X}
\end{gathered}
$$

and, since $\widetilde{\epsilon}=R_{k}^{2}$, the lemma follows.

We introduce the norm

$$
\begin{aligned}
& \|(W, F)\|=\sup _{k}\left[g\left(R_{k}, z\right) R_{k}^{4}\left(f\left|\nabla^{2} W\right|^{2}\right)^{1 / 2}+R_{k}^{3+1 / 2}\left(f_{J_{k}}\left|D_{x}^{1 / 2} D_{y} W(x, 0)\right|^{2}\right)^{1 / 2}\right. \\
& \left.+R_{k}^{3+1 / 2}|z|^{1 / 4}\left(f_{J_{k}}\left|D_{y} W(x, 0)\right|^{2}\right)^{1 / 2}\right]+|F(0)||z|^{-1 / 2} \\
& +\sup _{k}\left[g ( R _ { k } , z ) \left[R_{k}\left(f_{J_{k}}|F(x)-F(0)|^{2}\right)^{1 / 2}\right.\right. \\
& \left.\left.+R_{k}^{3+1 / 2}\left(f_{J_{k}}\left|D_{x}^{5 / 2}(F(x)-F(0))\right|^{2}\right)^{1 / 2}\right]\right] \\
& +\sup _{k}\left[g ( R _ { k } , z ) \left[\sup _{n}\left[R_{k}^{3} d_{k n}\left(f_{Q_{k n}}\left|\nabla^{2} W\right|^{2}\right)^{1 / 2}\right]\right.\right. \\
& \left.\left.+\sup _{n}\left[\frac{R_{k}^{3}}{\left|\log d_{k n}\right|}\left(f_{Q_{k n}}|\nabla W|^{2}\right)^{1 / 2}\right]\right]\right] \text {. }
\end{aligned}
$$

Note that we have collected in (11.11) all the coefficients of $|z|$ that appear in either (11.1) or on the right-hand sides of (5.6), (5.7).

From Lemma 11.1 we obtain: 
Lemma 11.2. Let $W, F, z$ be as in Lemma 11.1. Then

$$
\|(W, F)\| \leq C|z|^{\frac{\epsilon}{2}-\frac{1}{2}}|\log | z||\|Q\|_{X}
$$

where $C$ is a constant independent of $z$ and the source $Q$.

Remark 11.1. Both Lemma 5.1 and Lemmas 11.1,11.2 are valid for $\operatorname{Re} z \geq 0,|z|>$ 1 , provided we replace $|\log | z||$ (in (5.10), (11.2) and (11.12)) by $|\log (|z|+1)|$.

\section{Existence OF SOlutions}

We shall use Lemma 11.2 to establish existence and uniqueness for the system (2.1)-(2.6) for any $z$ in the region

$$
\Sigma_{0}: \operatorname{Re} z>0, \quad|z| \geq 1
$$

actually the proof can be extended to the larger region

$$
\operatorname{Re} z \geq-\frac{c}{|z|^{\frac{1+\epsilon}{2}}|\log (|z|+1)|}|\operatorname{Im} z|, \quad|z|>0
$$

for some $c>0$, but this will not be needed. We must however first extend Theorems $3.3,3.4$ to sources $Q=(a, b, c, \mu)$ with $\|Q\|_{X}<\infty$.

Consider first the case where $z \geq 1$.

Take smooth data $a, b, c, \mu$ with compact support and reduce the system (2.1)(2.6) to the system $(2.15)-(2.20)$ with vanishing $a, b, c$ and a new $\mu$, say $\widetilde{\mu}$, that belongs to $L^{2}(D)$ (this reduction was carried out in $\S 2$ ). By Theorems 3.3, 3.4, the reduced system has a unique solution $(\widetilde{W}, \widetilde{F})$, and this yields a solution $(W, F)$ to (2.1)-(2.6). By elliptic regularity (see [1] and [2]) the $\nabla^{j} W$ are uniformly bounded

in $\bar{D} \backslash B_{\rho}$ for $0 \leq j \leq 4$ and any $\rho>0$, where $B_{\rho}=\left\{x^{2}+y^{2}<\rho^{2}\right\}$. Also, by [6],

$$
W \sim A r^{3 / 2} \text { near the origin. }
$$

It follows that $\|(W, F)\|<\infty$.

We denote by $T_{z} Q$ the unique solution $(W, F)$ corresponding to the source $Q=$ $(a, b, c, \mu)$ which was thus established by means of Theorems 3.3, 3.4 for compactly supported smooth data.

Set

$$
\begin{aligned}
\mathcal{B}_{z}^{0}= & \{Q=(a, b, c, \mu) ; a, b, c \text { and } \mu \text { vanish } \\
& \text { if } \left.|x| \text { is large enough, and }\|Q\|_{X}<\infty\right\}, \\
\mathcal{B}_{z}= & \text { closure of } \mathcal{B}_{z}^{0} \text { under the norm }\|\|_{X} .
\end{aligned}
$$

Approximating by a sequence of cutoff functions, one can easily verify that the class $\mathcal{B}_{z}$ contains all sources with finite \|\|$_{X}$-norm which satisfy:

$$
\begin{gathered}
\lim _{|x| \rightarrow \infty} \mu(x, y, z)=0 \text { uniformly in } y, \\
\lim _{x \rightarrow-\infty} \partial_{x}^{i} a(x, z)=0 \quad(0 \leq i \leq 3), \quad \lim _{x \rightarrow-\infty} \partial_{x}^{j} b(x, z)=0 \quad(0 \leq j \leq 1), \\
\lim _{x \rightarrow-\infty} \partial_{x}^{k} c(x, z)=0 \quad(0 \leq k \leq 2) .
\end{gathered}
$$

Lemma 11.2 enables us to extend the solution of (2.1)-(2.6) (and uniquely so) to all $Q$ in $\mathcal{B}_{z}$ : 
Theorem 12.1. For any $z \in \sum_{0}$ and for any $Q=(a, b, c, \mu)$ in $\mathcal{B}_{z}$ there exists a unique solution of (2.1)-(2.6) having finite norm $\|(W, F)\|$, and

$$
\|(W, F)\| \leq C|z|^{\frac{\epsilon}{2}-\frac{1}{2}}|\log (|z|+1)|\|Q\|_{X},
$$

where $C$ is a constant independent of $Q, z$.

Proof. We have already proved the assertion for real $z, z \geq 1$. Let $\widetilde{z}$ be any complex number such that $\operatorname{Re} \widetilde{z}>0,|\widetilde{z}| \geq 1$, and suppose the assertion of the theorem is true for $\widetilde{z}$. We shall extend it to all nearby $z$. Writing $z=\widetilde{z}+(z-\widetilde{z})$ everywhere in (2.1)-(2.6) and moving the terms with $z-\widetilde{z}$ to the right-hand side, we can rewrite the equation

$$
(W, F)=T_{z}(Q)
$$

(this means, the solution $(W, F)$ corresponding to the source $Q$ ) in the form

$$
(W, F)=T_{\widetilde{z}}(Q)+(\widetilde{z}-z) T_{\widetilde{z}}(S(W, F))
$$

where

$$
S(W, F)=\left(F,-W_{y}(x, 0), 0, \Delta W\right) .
$$

Denote the right-hand side of (12.5) by $G(W, F)$. Then (12.5) means that ( $W, F)$ is a fixed point of the mapping $G$.

Notice that

$$
\left\|G\left(W_{1}, F_{1}\right)-G\left(W_{2}, F_{2}\right)\right\| \leq C|z|^{\frac{\epsilon}{2}-\frac{1}{2}}|\log (|z|+1)||\widetilde{z}-z|\left\|\left(W_{1}-W_{2}, F_{1}-F_{2}\right)\right\|
$$

and, therefore, if

$$
|\widetilde{z}-z|<\frac{\epsilon_{0}}{|z|^{\frac{\epsilon}{2}-\frac{1}{2}}|\log (|z|+1)|} \quad\left(\text { where } \epsilon_{0}<\frac{1}{C}\right),
$$

then $G$ is a contraction. Further, by Lemma 11.2, $G$ maps the ball of radius 1 and center $T_{\tilde{z}} Q$, i.e.,

$$
\left\{\left\|(W, F)-T_{\widetilde{z}} Q\right\| \leq 1\right\},
$$

into itself if $\epsilon_{0}$ is small enough. It follows that $G$ has a unique fixed point, and it belongs to this ball.

Applying the above result to any $\widetilde{z}>1$, we can proceed step-by-step to cover all of $\Sigma_{0}$ with $z$-neighborhoods for which (12.5) has a unique solution for any source $Q$. The proof of Theorem 12.1 also yields the estimate (12.4).

Remark 12.1. Note that $Q$ in (12.5) is the source $(a(x, z), b(x, z), c(x, z), \mu(x, y, z))$, whereas the norms in (12.6) are defined as in (11.11) with $z$ replaced by $\widetilde{z}$.

Set

$$
k(z)=|z|^{(1+\epsilon) / 2}|\log (|z|+1)| .
$$

Corollary 12.2. The solution established in Theorem 12.1 satisfies

$$
\|(W, F)\|_{Y}+\|(W, F)\|_{Z} \leq C k(z)\|Q\|_{X} .
$$


Indeed, Lemma 11.1 is applicable if $Q \in \mathcal{B}_{z}^{0}$ and $a, b, c, \mu$ are smooth enough (for then $(W, F)$ is smooth enough). By approximation, the estimate remains true for any $Q$ in $\mathcal{B}_{z}$.

We are now in a position to consider the system (1.8)-(1.15) directly. We define

$$
\begin{aligned}
\varphi(x, y, t) & =\frac{1}{2 \pi i} \int_{1-i \infty}^{1+i \infty} e^{z t} W(x, y, z) d z, \\
f(x, t) & =\frac{1}{2 \pi i} \int_{1-i \infty}^{1+i \infty} e^{z t} F(x, z) d z,
\end{aligned}
$$

where $(W, F)$ is the solution corresponding to the source

$$
Q_{z}=(a(\cdot, z), b(\cdot, z), c(\cdot, z), \mu(\cdot, z))
$$

and $a, b, c, \mu$ are the Laplace transforms of $a_{0}, b_{0}, c_{0}, \mu_{0}$, respectively. We assume that the data $a_{0}, b_{0}, c_{0}, \mu_{0}$ are such that

$$
\begin{gathered}
Q_{z} \in \mathcal{B}_{z} \quad \forall z, \operatorname{Re} z \geq 1, \\
k(z)\left\|Q_{z}\right\|_{X} \leq C \quad \forall z, \operatorname{Re} z \geq 1, \\
\int_{1-i \infty}^{1+i \infty} k(z)\left\|Q_{z}\right\|_{X} d|z|<\infty .
\end{gathered}
$$

We introduce the norm

$$
\|(\varphi, f)\|=\int_{1-i \infty}^{1+i \infty}\left[\|(W, F)\|_{Y}+\|(W, F)\|_{Z}\right] d|z|,
$$

where the norms on the right-hand side are defined in (5.6)-(5.9) and (11.1). By Lemma 11.1

$$
\|(W, F)\|_{Y}+\|(W, F)\|_{Z} \leq C k(z)\left\|Q_{z}\right\|_{X}
$$

and hence

$$
\|(\varphi, f)\| \leq C \int_{1-i \infty}^{1+i \infty} k(z)\left\|Q_{z}\right\|_{X} d|z| .
$$

The pair $(\varphi, f)$ is a generalized solution of $(1.8)-(1.15)$. Although it is not a classical solution, all the spatial derivatives which appear in (1.8)-(1.13) exist in a "strong sense"; in particular, if $R_{k} \approx 1$,

$$
\left(f_{S_{k}^{(1)}}\left|\nabla_{x y}^{4} \varphi(x, y, t)\right|^{2}\right)^{1 / 2}+\left(f_{S_{k}^{(1)}}\left|\nabla_{x y}^{2} \varphi(x, y, t)\right|^{2}\right)^{1 / 2} \leq C \int_{1-i \infty}^{1+i \infty} k(z)\left\|Q_{z}\right\|_{X} d|z| .
$$


By interpolation of some of the components of the norms in (11.11) one can show that

$$
|\varphi(x, y, t)| \leq C \int_{1-i \infty}^{1+i \infty} \frac{k(z)}{|z|}\left\|Q_{z}\right\|_{X} d|z|<\infty,
$$

and that $\varphi$ is a continuous function in $(x, y, t)$, for all $x \in \mathbb{R}, t \in \mathbb{R}, 0 \leq y \leq 1$, except possibly at $x=0, y=0, t \in \mathbb{R}$.

We next prove that $\varphi(x, y, t)=0$ if $t<0$.

For any large positive number $N$, introduce the half circle $\Gamma_{N}=\{|z-1|=$ $N, \operatorname{Re} z>1\}$ and the two arcs

$$
\Gamma_{N, \epsilon}=\Gamma_{N} \cap\left\{\frac{\pi}{2}-\epsilon<|\arg z|<\frac{\pi}{2}\right\}, \quad \epsilon>0 .
$$

Since $W(x, y, z)$ is analytic in $z$,

$$
\left(\int_{1-N i}^{1+N i}+\int_{\Gamma_{N, \epsilon}}+\int_{\Gamma_{N} \backslash \Gamma_{N, \epsilon}}\right) e^{z t} W(x, y, z) d z=0 .
$$

Also,

$$
\begin{aligned}
& \left|\int_{\Gamma_{N, \epsilon}} \ldots\right| \leq C \int_{\Gamma_{N, \epsilon}} \frac{k(z)}{|z|}\left\|Q_{z}\right\|_{X} \leq C \epsilon, \\
& \left|\int_{\Gamma_{N} \backslash \Gamma_{N, \epsilon}} \cdots\right| \leq C \int_{\Gamma_{N} \backslash \Gamma_{N \epsilon}} e^{-c \epsilon|z||t|} \rightarrow 0 \quad \text { if } N \rightarrow \infty .
\end{aligned}
$$

Using (12.8) we conclude that $\varphi(x, y, t)=0$ if $t<0$. Similarly one can show that $f(x, t)=0$ if $t<0$.

We summarize:

Theorem 12.2. If the data $a_{0}, b_{0}, c_{0}, \mu_{0}$ satisfy the conditions in (12.10) and if (1.15) holds, then there exists a unique generalized solution $(\varphi, f)$ of $(1.8)-(1.14)$ in the sense that $\|(\varphi, f)\|<\infty$ and it satisfies (1.8)-(1.14) in the a.e. sense; furthermore, the estimate (12.13) holds.

Note that the solution $(\varphi, f)$ was (uniquely) defined as the inverse Laplace transform of the unique solution $(W, F)$ established in Theorem 12.1. However, for any two classical solutions $\left(\varphi_{1}, f_{1}\right)$ and $\left(\varphi_{2}, f_{2}\right)$ of $(1.8)-(1.14)$, suitably bounded at $\infty$ and at the origin in both the $(x, y)$ and $t$ variables, the Laplace transforms $\left(W_{1}, F_{1}\right)$ and $\left(W_{2}, F_{2}\right)$ will have a finite norm \|\| and thus they will coincide, by Theorem 12.1 , so that $\varphi_{1}=\varphi_{2}, f_{1}=f_{2}$. Of course, we have not established here the existence of such classical solutions to (1.8)-(1.14).

\section{General Data}

In this section we extend the existence and uniqueness results of Theorem 12.2 for the linearized problem (1.8)-(1.14) to general data, namely, to data which do not necessarily satisfy the restrictions in (1.15). We assume for simplicity that

$$
\begin{array}{r}
\varphi_{0}=\partial_{y} \varphi_{0}=0 \text { if } y=1,-\infty<x<\infty \\
\text { and if } y=0,0<x<\infty .
\end{array}
$$


We also impose the consistency condition

$$
\frac{\partial^{2} \varphi_{0}}{\partial x^{2}}-\frac{\partial^{2} \varphi_{0}}{\partial y^{2}}=c_{0}(x) \quad \text { if } \quad y=0,-\infty<x<0 .
$$

We first wish to construct a solution $\left(\varphi_{1}, f_{1}\right)$ in the special case of data

$$
a_{0}(x, 0), b_{0}(x, 0), c_{0}(x, 0), \mu_{0}(x, y, 0) .
$$

Setting

$$
\varphi_{1}(x, y, t)=\varphi_{2}(x, y, t)+\varphi_{0}(x, y), \quad f_{1}(x, t)=f_{2}(x, t)+f_{0}(x),
$$

the pair $\left(\varphi_{2}, f_{2}\right)$ must satisfy the system:

$$
\begin{aligned}
& \frac{\partial}{\partial t}\left(\Delta \varphi_{2}\right)-\Delta^{2} \varphi_{2}=\mu_{0}(\cdot, 0)+\Delta^{2} \varphi_{0} \equiv \widetilde{\mu} \text { in } D \\
& \varphi_{2}=\frac{\partial \varphi_{2}}{\partial y}=0 \text { if } y=1,-\infty<x<\infty \\
& \varphi_{2}=\frac{\partial \varphi_{2}}{\partial y}=0 \text { if } y=0,0<x<\infty \\
& \frac{\partial f_{2}}{\partial t}+\beta_{0} \frac{\partial f_{2}}{\partial x}-\frac{\partial \varphi_{2}}{\partial x}=a_{0}(x, 0)-\beta_{0} \frac{\partial f_{0}}{\partial x}+\frac{\partial \varphi_{0}}{\partial x} \equiv \widetilde{a} \\
& \quad \text { if } y=0,-\infty<x<0, \\
& 3 \frac{\partial^{3} \varphi_{2}}{\partial x^{2} \partial y}+\frac{\partial^{3} \varphi_{2}}{\partial y^{3}}+\sigma \frac{\partial^{3} \varphi_{2}}{\partial x^{3}}-\frac{\partial^{3} \varphi_{2}}{\partial y \partial t} \\
& \quad=b_{0}(x, 0)-\left(3 \frac{\partial^{3} \varphi_{0}}{\partial x^{2} \partial y}+\frac{\partial^{3} \varphi_{0}}{\partial y^{3}}+\sigma \frac{\partial^{3} \varphi_{0}}{\partial x^{3}}\right) \\
& \equiv \widetilde{b} \text { if } y=0,-\infty<x<0, \\
& \frac{\partial^{2} \varphi_{2}}{\partial x^{2}}-\frac{\partial^{2} \varphi_{2}}{\partial y^{2}}=0 \text { if } y=0,-\infty<x<0, \\
& \varphi_{2}(x, y, 0)=0, \quad f_{2}(x, 0)=0
\end{aligned}
$$

in equation (13.9) we made use of the consistency condition (13.2).

We can solve this system using the Laplace transform. Indeed, denote by $W_{2}$, $F_{2}$ the Laplace transforms of $\varphi_{2}, f_{2}$, respectively. Then

$$
\begin{gathered}
z \Delta W_{2}-\Delta^{2} W_{2}=\frac{\widetilde{\mu}}{z} \text { in } D, \\
z F_{2}+\beta_{0} \partial_{x} F_{2}-\partial_{x} W_{2}=\frac{\widetilde{a}}{z} \text { if } y=0,-\infty<x<0,
\end{gathered}
$$

etc. By the method of $\S 12$ we know that for any $z$ in $\Sigma_{0}$ this problem has a unique solution $\left(W_{2}, F_{2}\right)$ satisfying

$$
\left\|\left(W_{2}, F_{2}\right)\right\| \leq \frac{C k(z)}{|z|}\|\widetilde{Q}\|_{X}
$$

where $\widetilde{Q}$ is the source

$$
\left(\frac{\widetilde{a}}{z}, \frac{\widetilde{b}}{z}, 0, \frac{\widetilde{\mu}}{z}\right)
$$


Notation. Denote by $\|Q(0)\|_{E}$ the right-hand side of (5.5) with $a=\widetilde{a}, b=\widetilde{b}, c=0$, $\mu=\widetilde{\mu}$ and with the term

$$
R_{k}^{3+1 / 2}\left(f_{J_{k}}|z|^{1 / 4}|\widetilde{b}|^{2}\right)^{1 / 2}
$$

removed (from (5.4)).

Clearly

$$
\|\widetilde{Q}\|_{X} \leq C|z|^{-3 / 4}\|Q(0)\|_{E}
$$

so that

$$
\left\|\left(W_{2}, F_{2}\right)\right\| \leq \frac{C k(z)}{|z|^{1+3 / 4}}\|Q(0)\|_{E} .
$$

Now define $\left(\varphi_{2}, f_{2}\right)$ by

$$
\begin{gathered}
\varphi_{2}=\frac{1}{2 \pi i} \int_{1-i \infty}^{1+i \infty} e^{z t} W_{2}(x, y, z) d z \\
f_{2}(x, t)=\frac{1}{2 \pi i} \int_{1-i \infty}^{1+i \infty} e^{z t} F_{2}(x, z) d z .
\end{gathered}
$$

Using the estimates $(13.11)$, we can proceed as in $\S 12$ to prove that $\varphi_{2}(x, y, t)=0$ and $f_{2}(x, t)=0$ if $t<0$, and $\left(\varphi_{2}, f_{2}\right)$ is a solution of (13.4)-(13.10) in $H^{2}(D)$; furthermore,

$$
\left\|\left(\varphi_{2}, f_{2}\right)\right\| \leq C\|Q(0)\|_{E} \int_{1-i \infty}^{1+i \infty} \frac{C k(z)}{|z|^{1+3 / 4}} \leq C\|Q(0)\|_{E}
$$

if we choose $\epsilon<\frac{1}{4}$, where the norm $\left\|\left(\varphi_{2}, f_{2}\right)\right\|$ is defined as in (12.11).

Next denote by $\left(\varphi_{3}, f_{3}\right)$ the solution of $(1.8)-(1.14)$ with $\varphi_{0}(x, y) \equiv 0, f_{0}(x) \equiv 0$ and data

$$
a_{0}(\cdot, t)-a_{0}(\cdot, 0), b_{0}(\cdot, t)-b_{0}(\cdot, 0), c_{0}(\cdot, t)-c_{0}(\cdot, 0), \mu_{0}(\cdot, t)-\mu_{0}(\cdot, 0) ;
$$

its existence and uniqueness were established by Theorem 12.2. In the Laplace transform notation, the sources for these data will be denoted by $Q_{z}(\cdot)-Q_{z}(0)$. We shall use the notation

$$
Q\left(\widehat{)-Q}(0)=Q_{z}(\cdot)-Q_{z}(0)\right.
$$

where the caret indicates the Laplace transform. Then we have

$$
\left\|\left(\varphi_{3}, f_{3}\right)\right\| \leq C \int_{1-i \infty}^{1+i \infty} k(z) \| Q\left(\cdot \widehat{)-Q}(0) \|_{X} d|z| .\right.
$$

We summarize: 
Theorem 13.1. Assume that (13.1), (13.2) hold and that

$$
\langle Q\rangle \equiv\|Q(0)\|_{E}+\int_{1-i \infty}^{1+i \infty} k(z) \| Q\left(\cdot \widehat{)-Q}(0) \|_{X} d|z|\right.
$$

is finite. Then the functions

$$
\varphi=\varphi_{0}+\varphi_{2}+\varphi_{3}, \quad f=f_{0}+f_{2}+f_{3}
$$

form the unique solution of (1.8)-(1.15) with finite norm $\left\|\left(\varphi-\varphi_{0}, f-f_{0}\right)\right\|$; moreover,

$$
\left\|\left(\varphi-\varphi_{0}, f-f_{0}\right)\right\| \leq C\langle Q\rangle
$$

where $C$ is a constant independent of the data.

Here the concept of uniqueness is in a sense similar to that for homogeneous data (as explained at the end of Section 12).

\section{Proof of Lemma 6.1}

We need to extend the a priori estimates of Section 4 to include estimates of lower order derivatives of $W$ and $F$ in terms of lower order derivatives of the source. To this purpose we define

$$
\partial_{y}^{-1} \psi(x, y)=\int_{y}^{\infty} \psi(x, y) d y, \quad \partial_{y}^{-n} \psi(x, y)=\partial_{y}^{-1}\left(\partial_{y}^{-(n-1)} \psi(x, y)\right)
$$

and

$$
\begin{aligned}
& \left\|\partial_{x}^{-\ell} \partial_{y}^{-n} \psi\right\|_{L^{2}\left(\mathbb{R}_{+}^{2}\right)}=\left\{\int_{0}^{\infty} d y \frac{\left|\partial_{y}^{-n} \hat{\psi}(k, y)\right|^{2}}{\left(1+|k|^{2}\right)^{\ell}} d k\right\}^{1 / 2}, \\
& \left\|\partial_{x}^{-\ell} a\right\|_{L^{2}(\mathbb{R})}=\left\{\int_{-\infty}^{\infty} \frac{|\hat{a}(k)|^{2}}{\left(1+|k|^{2}\right)^{\ell}} d k\right\}^{1 / 2},
\end{aligned}
$$

where $\hat{\psi}(k, y)$ and $\hat{a}(k)$ are the Fourier transform of $\psi(x, y)$ and $a(x)$ in $x$. 
In order to extend Lemma 4.3 we define

$$
\begin{aligned}
\|\mid(W, F)\|_{j}= & \left\{\int_{-\infty}^{\infty} d x \int_{0}^{\infty} d y\left[\left|\nabla^{4-j} W\right|^{2}+|\epsilon z|^{2}\left|\nabla^{2-j} W\right|^{2}\right]\right. \\
& +\int_{-\infty}^{\infty} d x\left[|\epsilon z|^{1 / 2}\left|\nabla^{3-j} W(x, 0)\right|^{2}+|\epsilon z|^{3 / 2}\left|\nabla^{2-j} W(x, 0)\right|^{2}\right. \\
& \left.+|\epsilon z|^{2}\left|\nabla^{\frac{3}{2}-j} W(x, 0)\right|^{2}+|\epsilon z|^{2+1 / 2}\left|\partial_{x}^{-j} \partial_{y} W(x, 0)\right|^{2}\right] \\
+ & \left.\int_{-\infty}^{\infty} d x\left[\left|D_{x}^{\frac{7}{2}-j} F(x)\right|^{2}+|z|\left|D_{x}^{3-j} F(x)\right|^{2}+|z|^{2}\left|D_{x}^{\frac{5}{2}-j} F(x)\right|^{2}\right]\right\}^{1 / 2}, \\
\|Q\|_{-j}= & \left\{\int_{-\infty}^{\infty} d x \int_{0}^{\infty} d y\left|\nabla^{-j} \mu(x, y)\right|^{2}\right. \\
+ & \int_{-\infty}^{\infty} d x\left[\left|\partial_{x}^{\frac{5}{2}-j} a\right|^{2}+\left|\partial_{x}^{\frac{1}{2}-j} b\right|^{2}+|\epsilon z|^{1 / 2}\left|\partial_{x}^{-j} b\right|^{2}+\left|\partial_{x}^{\frac{3}{2}-j} c\right|^{2}\right. \\
& \\
& \left.\left.+|\epsilon z|^{\frac{3}{2}}\left|\partial_{x}^{-j} c\right|^{2}\right]\right\}^{1 / 2},
\end{aligned}
$$

where $Q=(a, b, c, \mu)$.

Lemma 14.1. For any solution $(W, F)$ of (4.35)-(4.39) satisfying (4.42),

$$
\||(W, F)|\|_{-j} \leq C\|Q\|_{-j} \quad(j=0,1,2,3),
$$

where $C$ is a constant depending only on $R_{0}$.

This lemma coincides with Lemma 13.2 of [7] if $\epsilon=0$. The proof for $\epsilon>0$ is quite similar if we use the observations made in the proof of Lemma 3.2 of [7].

We shall apply Lemma 14.1 to prove:

Lemma 14.2. If $(W, F)$ is a solution to (4.35)-(4.39) and $W \in L^{2}\left(\mathbb{R}_{+}^{2}\right), F \in$ $L^{2}(\mathbb{R})$, then

$$
\begin{aligned}
& \left\|\nabla^{2} W\right\|_{L^{2}\left(\mathbb{R}_{+}^{2}\right)} \leq C\|W\|_{L^{2}\left(\mathbb{F}_{+}^{2}\right)}+C\|F\|_{L^{2}(\mathbb{R})}+C\|Q\|_{0}, \\
& \left\|\nabla^{4} W\right\|_{L^{2}\left(\mathbb{R}_{+}^{2}\right)} \leq C(1+\epsilon|z|)\|W\|_{L^{2}\left(\mathbb{R}_{+}^{2}\right)}+C\|F\|_{L^{2}(\mathbb{R})}+C\|Q\|_{0},
\end{aligned}
$$

and

$$
\begin{aligned}
& \left\|D^{7 / 2} F\right\|_{L^{2}(\mathbb{R})}+|z|^{1 / 2}\left\|D^{3} F\right\|_{L^{2}(\mathbb{R})}+|z|\left\|D^{5 / 2} F\right\|_{L^{2}(\mathbb{R})} \\
& \leq C(1+\epsilon|z|)\|W\|_{L^{2}\left(\mathbb{R}_{+}^{2}\right)}+C\|F\|_{L^{2}(\mathbb{R})}+C\|Q\|_{0},
\end{aligned}
$$

where $C$ is a constant independent of $z, Q$ and $(W, F)$.

Proof. We follow the same procedure as in the proof of Lemma 7.1 of [7]. We introduce cutoff functions $\eta_{k}(1 \leq k \leq 4)$ such that $\partial^{m} \eta_{k} / \partial y^{m}=0$ if $1 \leq m \leq 3$ and the support of each $\eta_{k}$ is contained in the set $\left\{\eta_{k-1}=1\right\}$. We define

$$
W_{k}=\eta_{k} W, \quad F_{k}=\eta_{k} F
$$


Then

$$
z \Delta W_{k}-\Delta^{2} W_{k}=\mu_{k}
$$

where each $\mu_{k}$ is a linear combination of

$$
\nabla^{3} W, \epsilon z \nabla W, \nabla^{2} W, \epsilon z W, W \text { and } \mu
$$

with coefficients that are derivatives of $\eta_{k}$ and thus vanish outside $\left\{\eta_{k-1}=1\right\}$. The corresponding boundary data for the $W_{k}, F_{k}$ will be denoted by $\left(a_{k}, b_{k}, c_{k}\right)$. The coefficient $a_{k}$ is a linear combination of

$$
F, D_{x} W \text { and } a,
$$

$b_{k}$ is a linear combination of

$$
\partial_{x} \partial_{y} W, \partial_{y} W, \partial_{x}^{2} F, \partial_{x} F, F \text { and } b,
$$

and $c_{k}$ is a linear combination of

$$
\partial_{x} W, W \text { and } c \text {. }
$$

We apply Lemma 14.1 with $j=3$ to $\left(W_{1}, F_{1}\right)$ and thus obtain an $L^{2}$ estimate of

$$
\nabla W_{1}, \epsilon|z| \nabla^{-1} W_{1} \text { and } \partial_{x} F_{1},|z|^{1 / 2} F_{1},|z| \partial_{x}^{-1 / 2} F_{1}
$$

by

$$
C\left\|\gamma_{1} W\right\|_{L^{2}\left(R_{+}^{2}\right)}+C\left\|\gamma_{1} F\right\|_{L^{2}(\mathbb{R})}+C\left\|\gamma_{1} Q\right\|_{0},
$$

where $\gamma_{1}$ is the characteristic function of the support of $\eta_{1}$. Next we apply Lemma 14.1 with $j=2$ to $\left(W_{2}, F_{2}\right)$ and, upon using also the previous estimate, obtain $L^{2}$ bounds on

$$
\partial_{x}^{3 / 2} F_{2},|z|^{1 / 2} \partial_{x} F_{2},|z| \partial_{x}^{1 / 2} F_{2}
$$

in terms of

$$
C(1+\epsilon|z|)\left\|\gamma_{1} W\right\|_{L^{2}\left(\mathbb{R}_{+}^{2}\right)}+C\left\|\gamma_{1} F\right\|_{L^{2}(\mathbb{R})}+C\left\|\gamma_{1} Q\right\|_{0} .
$$

In the next step we use Lemma 14.1 with $j=1$ to estimate the $L^{2}$ norms of

$$
\nabla^{3} W_{3}, \epsilon|z| \nabla W_{3}, \partial_{x}^{5 / 2} F,|z|^{1 / 2} \partial_{x}^{2} F_{3} \text { and }|z| \partial_{x}^{3 / 2} F_{3}
$$

by the right-hand side of (14.4). Finally, by applying Lemma 14.1 with $j=0$ (i.e., Lemma 4.3) we obtain an estimate of the $L^{2}$ norms of

$$
\nabla^{4} W_{4}, \partial_{x}^{7 / 2} F_{4},|z|^{1 / 2} \partial_{x}^{3} F_{4} \text { and }|z| \partial_{x}^{5 / 2} F_{4}
$$

by the right-hand sides of (14.4). From this estimate and the previous estimates on $\nabla^{2} W_{1}$ we obtain the assertions (14.2), (14.3).

Let $D_{0} \subset D_{1}$ be any two domains in $\mathbb{R}^{2}$ (not necessarily bounded), and set $D_{i}^{+}=D_{i} \cap\{y>0\}, \partial D_{i}^{+} \cap \mathbb{R}=\left\{(x, 0) ;(x, 0) \in \partial D_{i}^{+}\right\}$. We define $\|Q\|_{0, D_{1}^{+}}$by replacing in (4.40) the integration of $\mu$ over $\mathbb{R}_{+}^{2}$ by integration over $D_{1}^{+}$and the integration of the other data over $\{-\infty<x<\infty\}$ by integration over $\bar{D}_{1} \cap\{y=0\}$.

The proof of Lemma 14.2 establishes also the following local version: 
Lemma 14.3. Let $D_{0} \subset D_{1}$ be such that

$$
\operatorname{dist}\left(\overline{D_{0}^{+}}, \partial D_{1}^{+} \cap\{y>0\}\right) \geq c_{0}>0 .
$$

Suppose $(W, F)$ is a solution of (4.35)-(4.39) in $D_{1}^{+}$. Then

(i) If $W \in L^{2}\left(D_{1}\right)$ and $F \in L^{2}\left(\partial D_{1}^{+} \cap \mathbb{R}\right)$, then

$$
\begin{aligned}
& (1+\epsilon|z|)\left\|\nabla^{2} W\right\|_{L^{2}\left(D_{0}^{+}\right)}+\left\|\nabla^{4} W\right\|_{L^{2}\left(D_{0}^{+}\right)} \\
& +\left\|D_{x}^{7 / 2} F\right\|_{L^{2}\left(\partial D_{0}^{+} \cap \mathbb{R}\right)}+|z|^{1 / 2}\left\|D_{x}^{3} F\right\|_{L^{2}\left(\partial D_{0}^{+} \cap \mathbb{R}\right)}+|z|\left\|D_{x}^{5 / 2} F\right\|_{L^{2}\left(\partial D_{0}^{+} \cap \mathbb{R}\right)} \\
& \leq C(1+\epsilon|z|)\|W\|_{L^{2}\left(D_{1}^{+}\right)}+C\|F\|_{L^{2}\left(\partial D_{1}^{+} \cap \mathbb{R}\right)}+C\|Q\|_{0, D_{1}^{+}}
\end{aligned}
$$

where $C$ is a constant depending only on $c_{0}$ and on $D_{0}, D_{1}$;

(ii) If $W \in L^{\infty}\left(D_{1}^{+}\right)$and $F \in L^{\infty}\left(\partial D_{1}^{+} \cap \mathbb{R}\right)$, then

$$
\begin{aligned}
& (1+\epsilon|z|)\left\|\nabla^{2} W\right\|_{L^{2}\left(D_{0}^{+}\right)}+\left\|\nabla^{4} W\right\|_{L^{2}\left(D_{0}^{+}\right)} \\
& +\left\|D^{7 / 2} F\right\|_{L^{2}\left(\partial D_{0}^{+} \cap \mathbb{R}\right)}+|z|^{1 / 2}\left\|D_{x}^{3} F\right\|_{L^{2}\left(\partial D_{0}^{+} \cap \mathbb{R}\right)}+|z|\left\|D_{x}^{5 / 2} F\right\|_{L^{2}\left(\partial D_{0}^{+} \cap \mathbb{R}\right)} \\
& \leq C(1+\epsilon|z|) \sup _{D_{1}^{+}}|W|+C \sup _{\partial D_{1}^{+} \cap \mathbb{R}}|F|+C\|Q\|_{0, D_{1}^{+}},
\end{aligned}
$$

where $C$ is a constant depending only on $c_{0}$ and on $D_{0}, D_{1}$.

We define $\|\mu\|_{X_{2}\left(D_{1}^{+}\right)}$as in (5.2) with $S_{k}^{(2)}$ replaced by $D_{1}^{+}$.

We shall need an analog of Lemma 14.3 for the elliptic problem

$$
\begin{aligned}
z \Delta W-\Delta^{2} W=\mu & \text { in } \quad D_{1}^{+}, \\
W(x, 0)=\partial_{y} W(x, 0)=0 & \text { on } \quad \partial D_{1}^{+} \cap\{y=0\} .
\end{aligned}
$$

We begin with an auxiliary estimate.

Lemma 14.4. If $\sup |W|<\infty$ then the solution of (14.8), (14.9) satisfies, for all $(x, y) \in D_{0}^{+}$,

$$
|W(x, y)| \leq C y \sup _{D_{1}^{+}}|W|+\frac{C}{|z|}\|\mu\|_{X_{2}\left(D_{1}^{+}\right)} y|\log y|,
$$

where $C$ is a constant depending only on $D_{0}$ and $D_{1}$.

Proof. Consider first the case $\mu=0$ and let $W_{1}=\eta_{1} W$, where $\eta_{1}$ is a cutoff function in $D_{1}, \eta_{1}=1$ in $D_{0}$. Then

$$
z \Delta W_{1}-\Delta^{2} W_{1}=\mu_{1}
$$

where $\mu_{1}$ is a linear combination of

$$
z \nabla W, z W, \nabla^{3} W, \nabla^{2} W, \nabla W \text { and } W .
$$

We can represent $W_{1}$ by Green's function $\widetilde{V}_{R}\left(R=|z|^{1 / 2}\right)$ as in $[7,(8.12)]$, move the derivatives in (14.11) from $W$ into $\widetilde{V}_{R}$ (as in [7]), and then use the estimates on $D^{j} \widetilde{V}_{R}$ to obtain the conclusion (14.10) (with $\mu \equiv 0$ ). 
To establish (14.10) in the general case, where $\mu \not \equiv 0$, we introduce the special solution

$$
W_{p}(\xi, \eta)=\int_{\mathbb{R}_{+}^{2}} \widetilde{V}_{R}(\xi-\lambda, \eta, \theta) \mu(\lambda, \theta) d \lambda d \theta,
$$

where $R=|z|^{1 / 2}$. By the proof of Lemma 4.2,

$$
\left|W_{p}\right| \leq \frac{C}{|z|} y|\log y|\|\mu\|_{X_{2}\left(D_{1}^{+}\right)}
$$

Consider the function

$$
\widetilde{W}=W-W_{p}
$$

It satisfies

$$
\sup _{D_{1}^{+}}|\widetilde{W}| \leq \sup _{D_{1}^{+}}|W|+\frac{C}{z} y|\log y|\|\mu\|_{X_{2}\left(D_{1}^{+}\right)} .
$$

By the previous special case of Lemma 14.4,

$$
\sup _{D_{0}^{+}}|\widetilde{W}| \leq C y \sup _{D_{1}^{+}}|\widetilde{W}| .
$$

Substituting (14.12) into the right-hand side of (14.13), and noting also that

$$
\sup _{D_{0}^{+}}|W| \leq \sup _{D_{0}^{+}}|\widetilde{W}|+\frac{C}{|z|} y|\log y|\|\mu\|_{X_{2}\left(D_{1}^{+}\right)}
$$

(by the same proof as for (14.12)), we get (14.10).

We now state an extension of Lemma 14.3 to the system (14.8), (14.9).

Lemma 14.5. Let $W$ be a solution of (14.8), (14.9) such that $\sup |W|<\infty$. Then

$$
\begin{aligned}
& \left(\int_{D_{0}^{+}}\left|\nabla^{2} W\right|^{2}\right)^{1 / 2} \leq \frac{C}{d} \sup _{D_{1}^{+}}|W|+\frac{C}{|z| d}\|\mu\|_{X_{2}\left(D_{1}^{+}\right)}, \\
& \left(\int_{D_{0}^{+}}\left|\nabla^{4} W\right|^{2}\right)^{1 / 2} \leq \frac{C|z|}{d^{3}} \sup _{D_{1}^{+}}|W|+\frac{C}{d^{3}}\|\mu\|_{X_{2}\left(D_{1}^{+}\right)},
\end{aligned}
$$

where $d=|z|^{-1 / 2}$ and $C$ is a constant depending only on $D_{0}, D_{1}$.

Proof. We first estimate the derivatives of the special solution $W_{p}$ by using Lemma 4.1. Next we estimate the derivatives of $\widetilde{W}=W-W_{p}$ by truncation, analogously to the proof of Lemma 14.4; here the estimate (14.10) is used.

As a first step in the proof of Lemma 6.1 we shall prove:

Lemma 14.6. Under the assumptions of Lemma 6.1,

$$
\text { A }=0 \text {. }
$$


For simplicity we may assume that

$$
\left\|\left(W_{m}, F_{m}\right)\right\|_{Y}=1
$$

We also recall that

$$
R_{k_{m}} \approx 1, \quad\left|z_{m}\right| \rightarrow \infty
$$

so that

$$
R_{k_{m}}\left|z_{m}\right| \gg 1 .
$$

The proof of (14.16) depends on several lemmas.

In the next lemma we shall require only the inequality

$$
\left\|Q_{m}\right\|_{X} \ll \frac{1}{\left|z_{m}\right|^{\epsilon / 2}} .
$$

We shall need to work with the norms $\|(W, F)\|_{Y}$ taken not over the entire strip $D=\{-\infty<x<\infty, 0<y<1\}$ but only over rectangles

$$
D_{R}=\{-R<x<R, 0<y<1\} .
$$

We shall denote these norms by

$$
\|(W, F)\|_{Y, R} \text { or, briefly, by }\|(W, F)\|_{R} .
$$

Lemma 14.7. Suppose

$$
\left|W_{m}\right| \leq \frac{C}{\left|z_{m}\right| r^{3 / 2}} \quad \text { if } \frac{M}{\left|z_{m}\right|^{1 / 2}} \leq r \leq 1
$$

for some positive constants $M, C$. Then, for any constants $N_{2}>N_{1}>0$,

$$
\left|W_{m}\right|=o\left(\frac{1}{\left|z_{m}\right| r^{3 / 2}}\right) \text { for } \frac{N_{1}}{\left|z_{m}\right|^{1 / 2}} \leq r \leq \frac{N_{2}}{\left|z_{m}\right|^{1 / 2}}
$$

as $m \rightarrow \infty$.

Here, of course, $W_{m}$ depends on the parameter $z_{m}$.

Proof of Lemma 14.7. We first show that

$$
\|(W, F)\|_{R} \leq \frac{C}{|z|^{\epsilon / 2}} \text { for all } R<\frac{M}{|z|^{1 / 2}}
$$

and some constant $C$ (independent of $z$ ); here, for brevity, we have set $W=W_{m}$, $F=F_{m}, z=z_{m}$. Indeed, suppose (14.25) is not true, i.e.,

$$
\sup _{R<M /|z|^{1 / 2}}\|(W, F)\|_{R} \gg \frac{1}{|z|^{\epsilon / 2}},
$$

and denote by $R=R_{m}^{*}$ the value of $R$ at which the supremum is achieved. Let

$$
\widetilde{W}=\frac{W}{\|(W, F)\|_{R_{m}^{*}}}, \quad \widetilde{F}=\frac{F}{\mid(W, F) \|_{R_{m}^{*}}} .
$$

Then

$$
\begin{aligned}
& \|(\widetilde{W}, \widetilde{F})\|_{R} \leq 1 \text { for all } R<\frac{M}{|z|^{1 / 2}}, \\
& \|(\widetilde{W}, \widetilde{F})\|_{R_{m}^{*}}=1 .
\end{aligned}
$$


By (14.23), if $M|z|^{-1 / 2} \leq r \leq 1$ then

$$
\begin{aligned}
|\widetilde{W}| & \leq \frac{C}{|z| r^{3 / 2}} \frac{1}{\|(W, F)\|_{R_{m}^{*}}}=\frac{C}{|z|^{1-\epsilon / 2} r^{3 / 2}} \frac{1}{|z|^{\epsilon / 2}\|(W, F)\|_{R_{m}^{*}}} \\
& \leq \frac{C}{|z|^{1-\epsilon / 2} r^{3 / 2}} \quad(\text { by }(14.26)) .
\end{aligned}
$$

Without loss of generality we may assume that

$$
R_{m}^{*}\left|z_{m}\right|^{1 / 2} \rightarrow c>0 .
$$

Indeed, otherwise we have (for a subsequence) $R_{m}^{*}\left|z_{m}\right|^{1 / 2} \rightarrow 0$, and we can proceed as in the case (8.6) (c) to derive a contradiction to (14.28).

From $(14.27),(14.30)$ it follows that

$$
R_{k}^{2}\left(f_{S_{k}}\left|\nabla^{2} \widetilde{W}\right|^{2}\right)^{1 / 2} \leq \frac{C}{g\left(R_{k}, z\right)} \leq C\left(\frac{1}{|z|^{1 / 2}}\right)^{-2 \epsilon} R_{k}^{\frac{1}{2}+\epsilon} \text { if } R_{k}<\frac{M}{|z|^{1 / 2}} .
$$

Since $W(x, 0)=\partial_{y} W(x, 0)=0$ if $x>0$, we can apply the Poincaré inequality to deduce that

$$
|\widetilde{W}| \leq C\left(\frac{1}{|z|^{1 / 2}}\right)^{-2 \epsilon} r^{\frac{1}{2}+\epsilon} \text { if } r<\frac{M}{|z|^{1 / 2}} .
$$

Recall that $\widetilde{W}=\widetilde{W}_{m}(x, y)$ and $\widetilde{F}=\widetilde{F}_{m}(x)$, and introduce the functions

$$
\hat{W}_{m}(x, y)=\widetilde{W}_{m}\left(R_{m}^{*} x, R_{m}^{*} y\right), \hat{F}_{m}(x)=\widetilde{F}_{m}\left(R_{m}^{*} x\right) .
$$

From (14.29), (14.31) we see that

$$
\begin{gathered}
\left|\hat{W}_{m}(x, y)\right| \leq \frac{C\left|z_{m}\right|^{\frac{1}{4}+\frac{\epsilon}{2}}}{r^{3 / 2}} \text { if } 1<r<M_{m}\left|z_{m}\right|^{1 / 2}, \\
\left|\hat{W}_{m}(x, y)\right| \leq C\left|z_{m}\right|^{\frac{1}{4}+\frac{\epsilon}{2}} r^{\frac{1}{2}+\epsilon} \text { if } 0<r<1,
\end{gathered}
$$

for some $M_{m}$ such that $M_{m} \rightarrow M / c$ ( $c$ as in (14.30)) if $m \rightarrow \infty$. The functions $\hat{W}_{m} /\left|z_{m}\right|^{\frac{1}{4}+\frac{\epsilon}{2}}$ then converge to a function $\varphi$ which satisfies

$$
c \omega \Delta \varphi-\Delta^{2} \varphi=0 \text { in } \mathbb{R}_{+}^{2} \quad\left(\omega=\lim _{m \rightarrow \infty} \frac{z_{m}}{\left|z_{m}\right|}\right),
$$

with the boundary conditions (8.42)-(8.44) and

$$
|\varphi(x, y)| \leq \begin{cases}C r^{-3 / 2} & \text { if } r>1 \\ C r^{\frac{1}{2}+\epsilon} & \text { if } r<1 .\end{cases}
$$

By Theorem 1.1 of [7] we conclude that $\varphi \equiv 0$.

We can now use truncations, as in $\S 6$, to deduce that the left-hand side of (14.28) converges to zero as $m \rightarrow \infty$, which is a contradiction.

Having completed the proof of (14.25), we can now easily complete the proof of Lemma 14.7. Indeed, from (14.25) we get, by the Poincaré inequality,

$$
\left|W_{m}\right| \leq \frac{C}{\left|z_{m}\right|^{\epsilon / 2}} \frac{1}{g\left(r, z_{m}\right)} \leq \frac{C}{\left|z_{m}\right|^{\epsilon / 2}}\left(\frac{1}{\left|z_{m}\right|^{1 / 2}}\right)^{-2 \epsilon} r^{\frac{1}{2}+\epsilon}
$$


if $r<M /\left|z_{m}\right|^{1 / 2}$, so that the function

$$
\widetilde{W}_{m}(x, y)=W_{m}\left(\rho_{m} x, \rho_{m} y\right) \quad\left(\rho_{m}=\frac{1}{\left|z_{m}\right|^{1 / 2}}\right)
$$

satisfies

$$
\left|\widetilde{W}_{m}\right| \leq C\left|z_{m}\right|^{-1 / 4} r^{\frac{1}{2}+\epsilon} \quad \text { if } r<M
$$

On the other hand, from (14.23) we easily deduce that

$$
\left|\widetilde{W}_{m}\right| \leq C\left|z_{m}\right|^{-1 / 4} r^{\frac{1}{2}-\epsilon} \quad \text { if } M<r<\left|z_{m}\right|^{1 / 2} .
$$

Applying to $\varphi=\lim _{m \rightarrow \infty}\left(\left|z_{m}\right|^{1 / 4} \widetilde{W}_{m}\right)$ the same Liouville theorem as before (Theorem 1.1 of [7]), we conclude that $\varphi \equiv 0$, so that

$$
\left|z_{m}\right|^{1 / 4} \widetilde{W}_{m} \rightarrow 0 \quad \text { uniformly for } N_{1} \leq r \leq N_{2}
$$

if $m \rightarrow \infty$, and this is precisely the assertion (14.24).

In the next lemma we assume that (5.22) holds, so that

$$
\left\|Q_{m}\right\|_{X} \ll \frac{1}{\left|z_{m}\right|^{\epsilon / 2}|\log | z_{m}||} .
$$

Lemma 14.8. If $\left(W_{m}, F_{m}, Q_{m}\right)$ satisfy (14.17) and (14.32) (and $R_{k_{m}} \approx 1,\left|z_{m}\right| \rightarrow$ $\infty)$ then, for any $M>0$,

$$
\sup _{M\left|z_{m}\right|^{-1 / 2} \leq r \leq 1}\left[\left|z_{m}\right| r^{3 / 2}\left|W_{m}\right|\right] \rightarrow 0 \quad \text { if } m \rightarrow \infty .
$$

Since, by (6.16), (6.19),

$$
\lim _{m \rightarrow \infty} r^{3 / 2} z_{m} W_{m}=A \sin \frac{3 \theta}{2} \quad \text { if } r \approx 1, \delta_{0} \leq \theta \leq \pi \quad\left(\delta_{0}>0\right),
$$

we then conclude that $A=0$, and Lemma 14.6 follows.

Lemma 14.7 tells us roughly that $\left|z_{m}\right| r^{3 / 2}\left|W_{m}\right|$ becomes $o(1)$ if $r$ is decreased to $\approx\left|z_{m}\right|^{-1 / 2}$. Lemma 14.8 says that this "smallness" is maintained as we go back and increase $r$ to $r=1$. To prove it we shall work with averages of $W_{m}$ and derive for them an ODE, which will then be carefully studied in Lemma 15.1.

We introduce the average

$$
\varphi(r)=\int_{0}^{\pi} h_{0}(\theta, \epsilon) W(r, \theta) d \theta=\int_{0}^{\pi} h(\theta, r) W(r, \theta),
$$

where

$$
W=W_{m}, \quad z=z_{m}, \quad \epsilon=\frac{1}{r|z|^{1 / 2}}, \quad h_{0}(\theta, \epsilon)=h(\theta, r)
$$

$\varphi$ depends on $m$, i.e., $\varphi=\varphi_{m}$, but to simplify the notation we shall usually drop the index $m$.

The function $h$ is chosen to satisfy

$$
\epsilon^{2} h_{\theta \theta \theta \vartheta}=h_{\theta \vartheta}+\frac{9}{4} h, \quad 0<\theta<\pi,
$$

and

$$
h(0, r)=h_{\vartheta}(0, r)=h_{\theta}(\pi, r)=0 .
$$


Note that if $\epsilon=0$ then $\sin (3 \theta / 2)$ is a solution of (14.35) with $h(0, r)=h_{\vartheta}(\pi, r)=0$. As $\epsilon \rightarrow 0$ any solution of (14.35)-(14.36) develops a singular layer at $\vartheta=0$.

In Section 15 we shall construct $h$ explicitly and establish some estimates for its derivatives with respect to $\vartheta$ and $r$.

Setting

$$
\mathcal{L}=\frac{1}{r} \frac{\partial}{\partial r}\left(r \frac{\partial}{\partial r}\right)
$$

we compute

$$
\int_{0}^{\pi} \mathcal{L} W \cdot h d \vartheta=\mathcal{L}\left(\int_{0}^{\pi} h W d \vartheta\right)-\int_{0}^{\pi}(\mathcal{L} h) \cdot W d \vartheta-2 \int_{0}^{\pi} \frac{\partial h}{\partial r} \frac{\partial W}{\partial r} d \vartheta
$$

and

$$
\begin{aligned}
\int_{0}^{\pi} W_{\vartheta \vartheta} h d \vartheta & =\left[W_{\vartheta} h-W h_{\vartheta}\right]_{0}^{\pi}+\int_{0}^{\pi} W h_{\vartheta \vartheta} d \vartheta \\
& =h(\pi, r) W_{\vartheta}(r, \pi)+\int_{0}^{\pi} W h_{\vartheta \vartheta} d \vartheta
\end{aligned}
$$

by (14.36). Hence

$$
\int_{0}^{\pi} \Delta W \cdot h d \vartheta=\mathcal{L} \varphi+S_{1}(r)
$$

where

$$
\begin{gathered}
S_{1}(r)=\frac{h(\pi, r)}{r^{2}} W_{\vartheta}(r, \pi)+\frac{1}{r^{2}} \int_{0}^{\pi} W(r, \vartheta) h_{\theta \vartheta}(\theta) d \theta-\int_{0}^{\pi} Q_{1}(r, \vartheta) d \vartheta \\
Q_{1}=(\mathcal{L} h) \cdot W+2 \frac{\partial h}{\partial r} \frac{\partial W}{\partial r} .
\end{gathered}
$$

Next,

$$
\int_{0}^{\pi} \Delta^{2} W \cdot h d \vartheta=\int_{0}^{\pi} \mathcal{L}(\Delta W) \cdot h d \vartheta+\frac{1}{r^{2}} \int_{0}^{\pi} \frac{\partial^{2}}{\partial \vartheta^{2}}(\Delta W) \cdot h d \vartheta
$$

and

$$
\int_{0}^{\pi} \frac{\partial^{2}}{\partial \vartheta^{2}}(\Delta W) \cdot h d \vartheta=h(\pi, r) \frac{\partial}{\partial \vartheta} \Delta W(r, \pi)+\int_{0}^{\pi} \Delta W \cdot h_{\vartheta \vartheta} d \vartheta
$$

Since

$$
\mathcal{L}(\Delta W \cdot h)=\mathcal{L}(\Delta W) \cdot h+2 \frac{\partial}{\partial r} \Delta W \cdot \frac{\partial h}{\partial r}+\Delta W \cdot \mathcal{L}(h),
$$

we also have

$$
\int_{0}^{\pi} \mathcal{L}(\Delta W) \cdot h d \vartheta=\mathcal{L}\left(\int_{0}^{\pi} \Delta W \cdot h d \vartheta\right)-\int_{0}^{\pi} Q_{2}(r, \vartheta) d \vartheta
$$


where

$$
Q_{2}(r, \vartheta)=2 \frac{\partial \Delta W}{\partial r} \frac{\partial h}{\partial r}+\Delta W \cdot \mathcal{L}(h)
$$

We conclude that

$$
\begin{aligned}
\int_{0}^{\pi} \Delta^{2} W \cdot h & =\mathcal{L}\left(\int_{0}^{\pi} \Delta W \cdot h d \vartheta\right)-\int_{0}^{\pi} Q_{2} d \vartheta \\
& +\frac{1}{r^{2}}\left\{h(\pi) \frac{\partial}{\partial \vartheta} \Delta W(r, \pi)+\int_{0}^{\pi} \Delta W \cdot h_{\vartheta \vartheta} d \vartheta\right\} .
\end{aligned}
$$

Next, by (14.37)

$$
\mathcal{L}\left(\int_{0}^{\pi} \Delta W \cdot h\right)=\mathcal{L}^{2}(\varphi)+\mathcal{L}\left(S_{1}(r)\right) .
$$

Also

$$
\begin{aligned}
\int_{0}^{\pi} \Delta & W \cdot h_{\vartheta \vartheta}=\int_{0}^{\pi} \mathcal{L}(W) h_{\vartheta \vartheta}+\frac{1}{r^{2}} \int_{0}^{\pi} W_{\vartheta \vartheta} h_{\vartheta \vartheta} \\
= & \int_{0}^{\pi} \mathcal{L}(W) h_{\vartheta \vartheta}+\frac{1}{r^{2}}\left[W_{\vartheta}(r, \pi) h_{\vartheta \vartheta}(\pi, r)-W(r, \pi) h_{\vartheta \vartheta \vartheta}(\pi, r)\right] \\
& +\frac{1}{r^{2}} \int_{0}^{\pi} W h_{\vartheta \vartheta \vartheta \vartheta} .
\end{aligned}
$$

Using the last two relations in (14.41), we get an expression for $\int_{0}^{\pi} \Delta^{2} W \cdot h$, and subtracting from it $z \int_{0}^{\pi} \Delta W \cdot h$ (which is computed by (14.37) and (14.38)), we obtain

$$
\begin{array}{rl}
z \int_{0}^{\pi} \Delta W & h-\int_{0}^{\pi} \Delta^{2} W \cdot h \\
= & z\left[\mathcal{L}(\varphi)-\int_{0}^{\pi} Q_{1}+\frac{h(\pi, r)}{r^{2}} W_{\vartheta}(r, \pi)+\frac{1}{r^{2}} \int_{0}^{\pi} W h_{\vartheta \vartheta}\right] \\
& -\mathcal{L}^{2}(\varphi)-\mathcal{L}\left(S_{1}(r)\right) \\
& +\int_{0}^{\pi} Q_{2}-\frac{1}{r^{2}} h(\pi, r) \frac{\partial}{\partial \vartheta}(\Delta W)(r, \pi)-\frac{1}{r^{2}} \int_{0}^{\pi} \mathcal{L}(W) h_{\vartheta \vartheta} \\
& -\frac{1}{r^{4}}\left[W_{\vartheta}(r, \pi) h_{\vartheta \vartheta}(\pi, r)-W(r, \pi) h_{\vartheta \vartheta \vartheta}(\pi, r)\right]-\frac{1}{r^{4}} \int_{0}^{\pi} W h_{\vartheta \vartheta \vartheta \vartheta} .
\end{array}
$$


Clearly

$$
\begin{aligned}
& \frac{1}{r} \frac{\partial W}{\partial \vartheta}(r, \pi)=-\frac{\partial W}{\partial y} \\
& \frac{1}{r} \frac{\partial(\Delta W)}{\partial \vartheta}(r, \pi)=-\frac{\partial}{\partial y}(\Delta W)=-\frac{\partial^{3} W}{\partial x^{2} \partial y}-\frac{\partial^{3} W}{\partial y^{3}}
\end{aligned}
$$

whence

$$
\begin{aligned}
& \frac{1}{r^{2}} z h(\pi, r) W_{\vartheta}(r, \pi)-\frac{1}{r^{2}} h(\pi, r) \frac{\partial}{\partial \theta}(\Delta W)(r, \pi) \\
& \quad=-\frac{h(\pi, r)}{r}\left[2 \frac{\partial^{3} W}{\partial x^{2} \partial y}+\sigma \frac{\partial^{3} F}{\partial x^{3}}-b(x)\right] \quad(x=-r)
\end{aligned}
$$

by the boundary condition (2.5) for $W$. Using also (14.35), we get from (14.42)

$$
\begin{aligned}
& \int_{0}^{\pi} \mu h d \vartheta=z \int_{0}^{\pi} \Delta W \cdot h d \vartheta-\int_{0}^{\pi} \Delta^{2} W \cdot h d \vartheta \\
& =z \mathcal{L}(\varphi)-\frac{9 z}{4 r^{2}} \varphi-z \int_{0}^{\pi} Q_{1}(r, \vartheta) d \theta-\mathcal{L}^{2}(\varphi)-\mathcal{L}\left(S_{1}(r)\right) \\
& +\int_{0}^{\pi} Q_{2}(r, \vartheta) d \vartheta-\frac{1}{r^{2}} \int_{0}^{\pi} \mathcal{L}(W) h_{\vartheta \vartheta} d \vartheta \\
& -\frac{1}{r^{4}}\left[W_{\vartheta}(r, \pi) h_{\theta \vartheta}(\pi, r)-W(r, \pi) h_{\theta \vartheta \vartheta}(\pi, r)\right] \\
& -\frac{h(\pi, r)}{r}\left[2 \frac{\partial^{3} W}{\partial x^{2} \partial y}(r, \pi)+\sigma \frac{\partial^{3} F}{\partial x^{3}}-b(x)\right]
\end{aligned}
$$

We rewrite this equation in the form

$$
z\left(\mathcal{L}(\varphi)-\frac{9}{4 r^{2}} \varphi\right)=k(r, z) \equiv k(r)
$$

and set

$$
f(r, z)=z \varphi
$$

Then

$$
f_{r r}+\frac{1}{r} f_{r}-\frac{9}{4 r^{2}} f=k(r)
$$


where

$$
\begin{aligned}
& k=\sum_{j=1}^{11} k_{j}, \\
& k_{1}=\int_{0}^{2 \pi} \mu h d \vartheta, \quad k_{2}=-\frac{h(\pi, r)}{r} b(x), \quad k_{3}=\frac{h(\pi, r)}{r} \sigma \partial_{x}^{3} F(x), \\
& k_{4}=\frac{1}{r^{4}} W_{\theta}(r, \pi) h_{\vartheta \vartheta}(\pi, r), \quad k_{5}=-\frac{1}{r^{4}} W(r, \pi) h_{\vartheta \vartheta \vartheta}(\pi, r), \\
& k_{6}=\frac{h(\pi, r)}{r} 2 \frac{\partial^{3} W(r, \pi)}{\partial x^{2} \partial y}, \quad k_{7}=-\int_{0}^{\pi} Q_{2}(r, \vartheta) d \vartheta, k_{8}=z \int_{0}^{\pi} Q_{1}(r, \vartheta) d \vartheta, \\
& k_{9}=\frac{1}{r^{2}} \int_{0}^{\pi} \mathcal{L}(W) h_{\theta \vartheta} d \theta, \quad k_{10}=\mathcal{L}^{2}(\varphi), k_{11}=\mathcal{L}\left(S_{1}(r)\right) .
\end{aligned}
$$

We already know from $\S 6$ that

$$
r^{3 / 2} z_{m} W_{m} \rightarrow A \sin \frac{3 \theta}{2}+o(1)
$$

uniformly in compact subsets of $D$; further, the first $r$-derivative converges in the average sense (with respect to $\vartheta$ ) because

$$
\left|z_{m}\right|\left(f_{S_{m}^{(1)}}\left|\nabla^{2} W_{m}\right|^{2}\right)^{1 / 2} \text { and } \sup _{k} d_{k n}\left|z_{m}\right|\left(f_{Q_{k n}}\left|\nabla^{2} W_{m}\right|^{2}\right)^{1 / 2}
$$

are uniformly bounded. Consequently, if $A \neq 0$ then, for any $r_{2}>r_{1}>0$,

$$
\begin{aligned}
f(r) & =\frac{A_{m}}{r^{3 / 2}}(1+o(1)), \\
f^{\prime}(r) & =-\frac{3}{2} \frac{A_{m}}{r^{5 / 2}}(1+o(1))
\end{aligned}
$$

if $0<r_{1} \leq r \leq r_{2}<\infty$, where $A_{m} \rightarrow A$ if $m \rightarrow \infty$.

Observe that $r^{ \pm 3 / 2}$ are solutions of the homogeneous equation (14.46). Hence, by the variation of parameters formula,

$$
f_{0}(r) \equiv \frac{1}{3} r^{3 / 2} \int_{r_{0}}^{r} s^{-1 / 2} k(s) d s-\frac{1}{3} r^{-3 / 2} \int_{r_{0}}^{r} s^{5 / 2} k(s) d s+a r^{-3 / 2}+b r^{3 / 2}
$$

is the general solution of (14.46). We fix $r_{0}$ positive and small enough and try to choose $a$ and $b$ such that

$$
f_{0}\left(r_{0}\right)=f\left(r_{0}\right) \text { and } f_{0}^{\prime}\left(r_{0}\right)=f^{\prime}\left(r_{0}\right)
$$

i.e.,

$$
\begin{aligned}
& a r_{0}^{-3 / 2}+b r_{0}^{3 / 2}=f\left(r_{0}\right) \\
& -\frac{3}{2} a r_{0}^{-5 / 2}+\frac{3}{2} b r_{0}^{1 / 2}=f^{\prime}\left(r_{0}\right)
\end{aligned}
$$


thus we shall have $f_{0}(r) \equiv f(r)$. Substituting $f\left(r_{0}\right), f^{\prime}\left(r_{0}\right)$ from (14.48), we deduce that the last system of equations can be solved with $a \neq 0$ and $a=O(1), b=O(1)$, and

$$
\begin{aligned}
f(r) & =\frac{1}{3} r^{3 / 2} \int_{r_{0}}^{r} s^{-1 / 2} k(s) d s-\frac{1}{3} r^{-3 / 2} \int_{r_{0}}^{r} s^{5 / 2} k(s) d s \\
& +a r^{-3 / 2}+b r^{3 / 2} \text { for all } 0<r<r_{0}, \\
& a=a_{m} \rightarrow a_{*} \neq 0, b=b_{m} \rightarrow b_{*} \text { as } m \rightarrow \infty .
\end{aligned}
$$

Introduce

$$
c^{*}=\sup _{M /|z|^{1 / 2} \leq r \leq 1}\left\{\sup _{0 \leq \theta \leq \pi}\left[|z| r^{3 / 2}|W(r, \vartheta)|\right]+|z| r^{3 / 2}|F(r)|\right\} .
$$

Since $A \neq 0, c^{*}=c_{m}^{*}$ stays uniformly away from zero, say $c_{m}^{*} \geq c_{0}>0$.

Completion of the proof of Lemma 14.8. Denote by $r=R^{*}=R_{m}^{*}$ the value of $r$ where the "sup" is achieved in (14.50). Consider first the case where

$$
|z| r^{3 / 2}|F(r)| \leq C \sup _{0 \leq \vartheta \leq \pi}\left[|z| r^{3 / 2}|W(r, \vartheta)|\right] \text { at } r=R^{*},
$$

where $R^{*}=R_{m}^{*}, z=z_{m}, W=W_{m}$. Then by applying Lemma 14.7 to $W / c^{*}$ we deduce that

$$
R_{m}^{*} \gg \frac{M}{\left|z_{m}\right|^{1 / 2}} \text {. }
$$

Introduce

$$
\widetilde{W}(x, y)=W(R x, R y), \quad \widetilde{F}(x)=F(R x) \text { for } R|z| \gg 1 .
$$

Then

$$
\begin{aligned}
& R^{2} z \Delta \widetilde{W}-\Delta^{2} \widetilde{W}=\widetilde{\mu} \text { in } \mathbb{R}_{+}^{2}, \\
& R z \widetilde{F}+\beta_{0} \widetilde{F}_{x}-\widetilde{W}_{x}=\widetilde{a} \text { if } y=0,-\infty<x<0, \text { etc. }
\end{aligned}
$$

We apply Lemma 14.3(ii) with $\epsilon$ replaced by $R$ and $z$ replaced by $R z$. Taking $D_{0}$ and $D_{1}$ to be concentric circles of radii $\frac{1}{4}$ and $\frac{1}{2}$ respectively, and with center $(-1,0)$, we get

$$
\begin{aligned}
& R^{2}|z|\left\|\nabla^{2} \widetilde{W}\right\|_{L^{2}\left(D_{0}^{+}\right)}+\left\|\nabla^{4} \widetilde{W}\right\|_{L^{2}\left(D_{0}^{+}\right)}+\left\|D^{7 / 2} \widetilde{F}\right\|_{L^{2}\left(\partial D_{0}^{+} \cap \mathbb{R}\right)} \\
& +(R|z|)^{1 / 2}\left\|D_{x}^{3} F\right\|_{L^{2}\left(\partial D_{0}^{+} \cap \mathbb{R}\right)}+R|z|\left\|D_{x}^{5 / 2} F\right\|_{L^{2}\left(\partial D_{0}^{+} \cap \mathbb{R}\right)} \\
& \leq C R^{2}|z| \sup _{D_{1}^{+}}|\widetilde{W}|+C \sup _{\partial D_{1}^{+} \cap \mathbb{R}}|\widetilde{F}|+C\|\widetilde{Q}\|_{0, D_{1}^{+}} .
\end{aligned}
$$

From the definition of $c^{*}$ in (14.50) we get

$$
|\widetilde{W}(x, y)|=|W(R x, R y)| \leq \frac{c^{*}}{|z| R^{3 / 2}} \text { in } D_{1}^{+},
$$

provided

$$
\frac{2 M}{|z|^{1 / 2}}<R<\frac{1}{2} .
$$


The same bound holds for $\widetilde{F}$. Since further, by the definition of $\|Q\|_{X}$ and $g(R, z)$,

$$
\|\widetilde{Q}\|_{0, D_{1}^{+}} \leq C R^{1 / 2} R^{\epsilon}\|Q\|_{X}
$$

we see that the right-hand side of (14.53) is bounded by

$$
R^{1 / 2}\left(C c^{*}+C\|Q\|_{X} R^{\epsilon}\right), \text { or by } C R^{1 / 2} c^{*}
$$

by (14.32). Therefore (14.53) yields

$$
\begin{aligned}
& |z| R^{\frac{3}{2}+2}\left(f_{S_{R}^{(1)}}\left|\nabla^{2} W\right|^{2}\right)^{1 / 2} \leq C c^{*}, \\
& R^{\frac{3}{2}+2}\left(f_{S_{R}^{(1)}}\left|\nabla^{4} W\right|^{2}\right)^{1 / 2} \leq C c^{*}, \\
& R^{\frac{3}{2}+1}\left(f_{J_{R}}\left|D_{x}^{7 / 2} F\right|^{2}\right)^{1 / 2} \leq C c^{*}, \\
& |z| R^{\frac{3}{2}+1}\left(f_{J_{R}}\left|D_{x}^{5 / 2} F\right|^{2}\right)^{1 / 2} \leq C c^{*} ;
\end{aligned}
$$

here $S_{R}^{(1)}$ means $S_{k}^{(1)}$ with $R_{k} \approx R$, and $J_{R}$ is the corresponding $J_{k}$. By (14.50)

$$
|z| R^{3 / 2}\left(f_{S_{R}^{(1)}}|W|^{2}\right)^{1 / 2} \leq c^{*},
$$

and then, by interpolation,

$$
|z| R^{\frac{3}{2}+1}\left(f_{S_{R}^{(1)}}|\nabla W|^{2}\right)^{1 / 2} \leq C c^{*} .
$$

Similarly, by Lemma 14.4,

$$
|z| R^{3 / 2}\left(f_{Q_{k n}}|W|^{2}\right)^{1 / 2} \leq C c^{*} d_{k n}
$$


(here we make full use of (14.32), i.e., including the $\log \left|z_{m}\right|$ factor), and by Lemma 14.5 and interpolation we then get

$$
\begin{aligned}
& |z| R^{\frac{3}{2}+\frac{1}{2}}\left(f_{Q_{k n}}|\nabla W|^{2}\right)^{1 / 2} \leq C c^{*}, \\
& |z| R^{\frac{3}{2}+1} d_{k n}\left(f_{Q_{k n}}\left|\nabla^{2} W\right|^{2}\right)^{1 / 2} \leq C c^{*}, \\
& R^{\frac{3}{2}-1} d_{k n}^{3}\left(f_{Q_{k n}}\left|\nabla^{4} W\right|^{2}\right)^{1 / 2} \leq C c^{*},
\end{aligned}
$$

where $S_{R}^{(2)}$ is $S_{k}^{(2)}$ with $R_{k} \approx R$, and the $Q_{k n}$ form a partition of $S_{R}^{(2)}$ into rectangles.

Having proved (14.55)-(14.57) for any $R$ which satisfies (14.54), we can see upon recalling (14.51) that we are in a situation similar to that of

$$
R_{k_{m}} \gg\left|z_{m}\right|^{-1 / 2}
$$

studied in earlier sections. Indeed, we are in a situation similar to case (8.6)(a). It will suffice to consider the case where the $W$ 's dominate the $(W, F)$-norm (if the $F$ 's dominate the $(W, F)$-norm then we proceed precisely as in case (8.6)(a) in Section $9)$. We then have

$$
z_{m} W_{m} \approx \frac{\hat{A}}{r^{3 / 2}} \sin \frac{3 \vartheta}{2} \quad \text { at } r=R_{m}^{*}
$$

where $\hat{A}$ is a constant independent of $m$. Since by (14.51)

$$
\sup _{0 \leq \vartheta \leq \pi}\left[\left|z_{m}\right| r^{3 / 2}\left|W_{m}(r, 0)\right|\right] \approx \rho c^{*} \quad \text { at } r=R_{m}^{*},
$$

where $\rho$ is a constant independent of $m$, it follows that

$$
\hat{A} \approx \frac{2 \rho c^{*}}{\pi}
$$

so that

$$
z_{m} W_{m} \approx \frac{2 \rho}{\pi} \frac{c^{*}}{r^{3 / 2}} \sin \frac{3 \vartheta}{2} \quad \text { at } r=R_{m}^{*}
$$

To continue with the proof of Lemma 14.8 we need the following result.

Lemma 14.9. Set

$$
f_{j}(r)=\frac{1}{3} r^{3 / 2} \int_{r_{0}}^{r} s^{-1 / 2} k_{j}(s) d s, \quad \widetilde{f}_{j}(r)=\frac{1}{3} r^{-3 / 2} \int_{r_{0}}^{r} s^{5 / 2} k_{j}(s) d s .
$$

Then, for any $M>0$,

$$
\left|f_{j}(r)\right|+\left|\widetilde{f}_{j}(r)\right| \leq \frac{C c_{*}}{r^{3 / 2}} \frac{1}{\left(r|z|^{1 / 2}\right)^{\delta}} \quad \text { if } \frac{M}{|z|^{1 / 2}} \leq r \leq 1
$$

for $1 \leq j \leq 11$ and some $\delta>0$. 
Suppose the lemma is true. Then we can complete the proof of Lemma 14.8 as follows:

By Lemma 14.9 and (14.49) we see that, at $r=R_{m}^{*}, a_{*} r^{-3 / 2}$ is the dominant term of $f(r)$, i.e.,

$$
f(r) \approx \frac{a_{*}}{r^{3 / 2}}
$$

at $r=R_{m}^{*}$. By comparison with (14.59) it follows that $a_{*}=\rho c^{*}$. Further, by Lemma 14.9, the relation (14.60) continues to hold for smaller $r$ 's up to $r=$ $M /\left|z_{m}\right|^{1 / 2}$ if $M$ is a sufficiently large positive constant (independent of $m$ ). Consequently

$$
\sup _{0 \leq \vartheta \leq \pi}\left|z_{m} W_{m}(r, \vartheta)\right| \geq \frac{1}{2} \frac{\rho c^{*}}{r^{3 / 2}} \text { if } r=\frac{M}{\left|z_{m}\right|^{1 / 2}} .
$$

If we start the previous analysis with such an $M$ (in the definition (14.50) of $c^{*}$ ), then we also have

$$
\left|z_{m} W_{m}(r, \vartheta)\right| \leq \frac{C c^{*}}{r^{3 / 2}} \quad \text { if } \frac{M}{\left|z_{m}\right|^{1 / 2}} \leq r \leq 1 .
$$

The last two inequalities are in contradiction to the assertion of Lemma 14.7.

So far we have assumed that (14.51) is satisfied. It therefore remains to consider the case where

$$
|z| r^{3 / 2}|F(r)| \gg \sup _{0 \leq \theta \leq \pi}\left[|z| r^{3 / 2}|W(r, \vartheta)|\right] \text { at } r=R_{m}^{*}, \text { for } z=z_{m}, W=W_{m} .
$$

We shall first consider the subcase where

$$
\frac{2 M}{\left|z_{m}\right|^{1 / 2}}<R_{m}^{*}<\frac{1}{2}
$$

and introduce

$$
\widetilde{W}(x, y)=W\left(R_{m}^{*} x, R_{m}^{*} y\right), \quad \widetilde{F}(x)=F\left(R_{m}^{*} x\right) .
$$

We apply Lemma 14.3 with $D_{0}, D_{1}$ as above to appropriate truncations $\hat{W}=$ $\widetilde{W} \xi(x, y), \hat{F}=\widetilde{F} \xi(x, 0)$ and obtain (14.53) for $\hat{W}, \hat{F}$, and then, by Sobolev's inequality, we get

$$
\begin{aligned}
& R^{2}|z| \sup _{D_{0}^{+}}|\widetilde{W}|+|z| R \sup |\widetilde{F}| \\
& \quad \leq C R^{2}|z| \sup _{D_{1}^{+}}|\widetilde{W}|+C \sup _{\partial D_{1}^{+} \cap \mathbb{R}}|\widetilde{F}|+C\|\widetilde{Q}\|_{0, D_{1}^{+}}
\end{aligned}
$$

with $R=R_{m}^{*}$ and slightly different $D_{0}, D_{1}$; the detailed proof of (14.63) is given at the end of this section. Using (14.61) we then get

$$
|z| R^{5 / 2} \sup _{D_{0}^{+}}|\widetilde{W}|+c^{*} \leq C|z| R^{5 / 2} \sup _{D_{1}^{+}}|\widetilde{W}|+\frac{C c^{*}}{|z|}+\frac{C}{R^{1 / 2}}\|\widetilde{Q}\|_{0, D_{1}^{+}}+o(1)
$$

and the term with $\widetilde{Q}$ is $o(1)$, by (5.11). It follows (by (14.61)) that $c^{*}=o(1)$, a contradiction.

Next we consider the case

$$
\frac{M}{\left|z_{m}\right|^{1 / 2}} \leq R_{m}^{*} \leq \frac{2 M}{\left|z_{m}\right|^{1 / 2}} .
$$


We cannot repeat the previous argument, since $D_{1}$ goes outside the region where the supremum $c^{*}$ is taken and, consequently, Lemma 14.3 cannot be used in the same way. Proceeding differently, we recall that $\|(W, F)\|_{Y}=1$. Since we are in the case where $|z|^{1 / 2}|F(0)|$ is not a dominating term in the norm $\|(W, F)\|_{Y}$, it follows that

$$
|F(0)| \leq \frac{C}{|z|^{1 / 2}}
$$

and thus

$$
R|z||F(0)| \leq C \quad \text { if } \frac{M}{|z|^{1 / 2}} \leq R \leq \frac{2 M}{|z|^{1 / 2}}
$$

By the definition of $\|(W, F)\|_{Y}$ (see (5.6)-(5.9)) we have

$$
R^{\frac{1}{2}+\epsilon}|z|\left(f_{J_{k}}|F-F(0)|^{2}\right)^{1 / 2} \leq C
$$

and

$$
R^{-\frac{1}{2}+\epsilon}\|W\|_{L^{\infty}\left(S_{k}\right)} \leq C|\log R|
$$

for $R$ in the above range; here $J_{k}$ and $S_{k}$ have diameters $\approx R$. It follows that

$$
\left(f_{J_{k}}|F|^{2}\right)^{1 / 2} \leq \frac{C}{R|z|} \text { and }\|W\|_{L^{\infty}\left(S_{k}\right)} \leq \frac{C|\log R|}{R^{-\frac{1}{2}+\epsilon}} .
$$

Applying (14.6) of Lemma 14.3 to $\left(W\left(R_{m}^{*} x, R_{m}^{*} y\right), F\left(R_{m}^{*} x\right)\right)$ with $\epsilon$ and $z$ replaced by $R_{m}^{*}$ and $R_{m}^{*} z$ and using (14.32), we find (cf. (14.53) and the derivation of (14.63) below) that

$$
|z| R|F(R)| \leq \frac{C}{R|z|}+C R^{2}|z| \frac{|\log R|}{R^{-\frac{1}{2}+\epsilon}}
$$

at $R=R_{m}^{*}$. Hence

$$
|z| R^{3 / 2}|F(R)| \leq C R^{1-\epsilon}|\log R|
$$

and again $c^{*}=o(1)$, which is a contradiction.

Finally it remains to consider the case (14.61) with $\frac{1}{2} \leq R_{m}^{*}<1$. Since $A \neq 0$, the right-hand side of (14.61) is $\geq$ const. $>0$ at $r=R_{m}^{*}$, and so

$$
\left|z_{m}\right|\left|F\left(R_{m}^{*}\right)\right| \rightarrow \infty \text { if } m \rightarrow \infty \text {. }
$$

On the other hand, the previous argument ((14.61) with $M\left|z_{m}\right|^{-1 / 2} \leq R_{m}^{*} \leq$ $\left.2 M\left|z_{m}\right|^{-1 / 2}\right)$ shows that

$$
\left|z_{m}\right| R_{m}^{*}\left|F\left(R_{m}^{*}\right)\right| \leq \frac{C}{R_{m}^{*}\left|z_{m}\right|}+C\left|z_{m}\right| \sup |W|
$$

where the "sup" is taken over $\frac{1}{4}<r<2$. Since the right-hand side is bounded, we get

$$
\left|z_{m}\right|\left|F\left(R_{m}^{*}\right)\right| \leq C
$$

a contradiction. 
In the next section we shall prove Lemma 14.9 , after which we shall briefly complete the proof of Lemma 6.1 and also indicate the proof of Lemma 8.1.

Proof of (14.63). If we choose $\xi$ such that $\partial_{y}^{j} \xi(x, 0)=0(1 \leq j \leq 3)$, then $(\hat{W}, \hat{F})$ satisfies

$$
R^{2} z \Delta \hat{W}-\Delta^{2} \hat{W}=\xi \widetilde{\mu}+\left(R^{2} z \partial \widetilde{W}+\partial^{3} W\right) \partial \xi+\cdots
$$

with

$$
\hat{W}(x, 0)=\partial_{y} \hat{W}(x, 0)=0 \text { if } x>0,
$$

and

$$
\begin{gathered}
z R \hat{F}+\beta_{0} \hat{F}_{x}-\hat{W}_{x}=\xi \widetilde{a}+\xi_{x}\left(\beta_{0} \widetilde{F}-\widetilde{W}\right), \\
3 \partial_{x}^{2} \partial_{y} \hat{W}+R^{2} z \hat{W}_{y}+\partial_{y}^{2} \hat{W}-\partial^{3} \hat{F}=\xi \widetilde{b}+\partial_{x} \partial_{y} \widetilde{W} \cdot \xi_{x}+3 \partial_{y} \widetilde{W} \cdot \xi_{x x}, \\
\hat{W}_{x x}-\hat{W}_{y y}=\xi \widetilde{c}+2 \partial_{x} \widetilde{W} \cdot \xi_{x}+\widetilde{W} \xi_{x x}
\end{gathered}
$$

if $y=0, x<0$.

Notice that the source contains terms like $R^{2} z \partial \widetilde{W}, \partial^{3} \widetilde{W}, F, R^{2} z \widetilde{W}, \partial^{2} \widetilde{W}, \partial^{2} \widetilde{F}$ and lower order derivatives, but not $R z \widetilde{F}$. We can now follow the procedure used in the proof of Lemma 14.2 to estimate derivatives successively. For instance, in the first step we obtain estimates for

$$
R^{2}|z|\left(\int_{\mathbb{R}_{+}^{2}}|\nabla \hat{W}|^{2}\right)^{1 / 2}, \quad\left(\int_{-\infty}^{0}|\partial \hat{F}|^{2}\right)^{1 / 2}
$$

in terms of

$$
R^{2}|z| \sup _{D_{1}^{+}}|\widetilde{W}|, \quad \sup _{\partial D_{1}^{+} \cap \mathbb{R}}|\widetilde{F}| .
$$

Thus, although $\partial^{2} \widetilde{F}$ is originally bounded only in $H^{-2}$ (since we originally have bounds only on $\sup |\widetilde{W}|$ and $\sup |\widetilde{F}|)$, we now get an estimate on $\partial \hat{F}$ in $H^{1}$.

We continue with three more iterations, and finally conclude that

$$
\begin{gathered}
R^{2}|z|\left\|\nabla^{2} \hat{W}\right\|_{L^{2}\left(\mathbb{R}_{+}^{2}\right)}+\left\|\nabla^{4} \hat{W}\right\|_{L^{2}\left(\mathbb{R}_{+}^{2}\right)}+\left\|D^{7 / 2} \hat{F}\right\|_{L^{2}(\mathbb{R})} \\
+(R|z|)^{1 / 2}\left\|D^{3} \hat{F}\right\|_{L^{2}(\mathbb{R})}+R|z|\left\|D^{5 / 2} \hat{F}\right\|_{L^{2}(\mathbb{R})} \\
\leq C R^{2}|z| \sup _{D_{1}^{+}}|\widetilde{W}|+C \sup _{\partial D_{1}^{+} \cap \mathbb{R}}|\widetilde{F}|+C\|\widetilde{Q}\|_{0, D_{1}^{+}}
\end{gathered}
$$

which is essentially (14.53) for $(\hat{W}, \hat{F})$. Actually we need to choose a different cutoff function $\xi$ for each of the four iterations, but this does not change the final estimate.

Since $\hat{F}$ and $\hat{W}$ are compactly supported,

$$
\begin{aligned}
\|\hat{F}\|_{L^{\infty}(\mathbb{R})} & \leq C\left\|D^{5 / 2} \hat{F}\right\|_{L^{2}(\mathbb{R})}, \\
\|\hat{W}\|_{L^{\infty}\left(\mathbb{R}_{+}^{2}\right)} & \leq C\left\|\nabla^{2} \hat{W}\right\|_{L^{2}(\mathbb{R})} .
\end{aligned}
$$

Noting that $\hat{W}=\widetilde{W}, \hat{F}=\widetilde{F}$ in $D_{0}^{+}$and on $\partial D_{0}^{+} \cap \mathbb{R}$ respectively, we get (14.63). 
15. Completion of proof of Lemma 6.1; proof of Lemma 8.1

We begin the proof of Lemma 14.9 by first constructing a solution $h$ to (14.35), (14.36) having suitable behavior.

The general solution of (14.35) has the form

$$
h_{0}(\theta, \epsilon)=A_{\epsilon} e^{r_{1}^{+} \vartheta}+B_{\epsilon} e^{r_{1}^{-} \vartheta}+C_{\epsilon} e^{r_{2}^{+} \vartheta}+D_{\epsilon} e^{r_{2}^{-} \vartheta}
$$

where

$$
\begin{aligned}
& r_{1}^{+}=\left(\frac{1+\left(1+9 \epsilon^{2}\right)^{1 / 2}}{2 \epsilon^{2}}\right)^{1 / 2}=\frac{1}{\epsilon}\left(1+O\left(\epsilon^{2}\right)\right), \text { a positive real number, } \\
& r_{2}^{r}=-r_{1}^{+}=-\frac{1}{\epsilon}\left(1+O\left(\epsilon^{2}\right)\right), \\
& r_{2}^{+}=\left(\frac{1-\left(1+9 \epsilon^{2}\right)^{1 / 2}}{2 \epsilon^{2}}\right)^{1 / 2}=\frac{3 i}{2}\left(1+O\left(\epsilon^{2}\right)\right), \text { pure imaginary, } \\
& r_{2}^{-}=-r_{2}^{+}=-\frac{3 i}{2}\left(1+O\left(\epsilon^{2}\right)\right) .
\end{aligned}
$$

Setting for brevity $h_{0}(\vartheta)=h_{0}(\vartheta, \epsilon)$ and

$$
\omega_{\epsilon}=\left|r_{2}^{+}\right|=\frac{3}{2}\left(1+O\left(\epsilon^{2}\right)\right), \quad \lambda_{\epsilon}=r_{1}^{+}=\frac{1}{\epsilon}\left(1+O\left(\epsilon^{2}\right)\right),
$$

we can rewrite $h_{0}(\theta)$ in the form

$$
h_{0}(\theta)=A_{\epsilon} \sin \left(\omega_{\epsilon} \theta\right)+B_{\epsilon} \cos \left(\omega_{\epsilon} \theta\right)+C_{\epsilon} \sinh \left(\lambda_{\epsilon} \theta\right)+D_{\epsilon} \cosh \left(\lambda_{\epsilon} \vartheta\right) .
$$

The boundary conditions $h_{0}(0)=0, h_{0}^{\prime}(0)=0$ together with the choice $A_{\epsilon}=1$ give $C_{\epsilon}=-\omega_{\epsilon} / \lambda_{\epsilon}$ and $D_{\epsilon}=-B_{\epsilon}$, and the condition $h_{0}(\pi)=0$ then becomes

$$
B_{\epsilon}=\frac{\frac{\omega_{\epsilon}}{\lambda_{\epsilon}} \sinh \left(\lambda_{\epsilon} \pi\right)-\sin \left(\omega_{\epsilon} \pi\right)}{\cos \left(\omega_{\epsilon} \pi\right)-\cosh \left(\lambda_{\epsilon} \pi\right)} \text {. }
$$

Note that

$$
\frac{\omega_{\epsilon}}{\lambda_{\epsilon}}=\frac{3}{2} \epsilon\left(1+O\left(\epsilon^{2}\right)\right)
$$

and

$$
B_{\epsilon}=-\frac{\omega_{\epsilon}}{\lambda_{\epsilon}}\left(1+O\left(e^{-2 \lambda_{\epsilon} \pi}\right)\right)=\frac{3}{2} \epsilon\left(1+O\left(\epsilon^{2}\right)\right) .
$$

Using the relation $\sinh x-\cosh x=-e^{-x}$, we can then write

$$
h_{0}(\vartheta, \epsilon)=\sin \left(\omega_{\epsilon} \vartheta\right)+\frac{\omega_{\epsilon}}{\lambda_{\epsilon}}\left(e^{-2 \lambda_{\epsilon} \vartheta}-\cos \left(\omega_{\epsilon} \vartheta\right)\right)+\rho_{\epsilon},
$$

where $\rho_{\epsilon}$ is exponentially small together with its derivatives. Thus

$$
h_{0}(\vartheta, \epsilon) \approx \sin \frac{3 \vartheta}{2}+\frac{3 \epsilon}{2}\left(e^{-\frac{2 \vartheta}{\epsilon}}-1\right),
$$

where the second term is a boundary layer at $\vartheta=0$.

Lemma 15.1. The function $h(\vartheta, r) \equiv h_{0}(\vartheta, \epsilon)$ satisfies (15.1) with $\rho_{\epsilon}$ exponentially small together with its derivatives in $(\vartheta, \epsilon)$; consequently,

$$
\begin{aligned}
& \frac{\partial^{j} h}{\partial r^{j}}=O\left(\frac{\epsilon}{r^{j}}\right), \frac{\partial^{j+1} h}{\partial r^{j} \partial \vartheta}=O\left(\frac{1}{r^{j}}\right) \quad \text { for } j \geq 1, \\
& \frac{\partial h}{\partial \vartheta}=O(1), \quad \text { for } 0 \leq \vartheta \leq \pi
\end{aligned}
$$


and, for any $\delta>0$,

$$
\frac{\partial^{k} h}{\partial \vartheta^{k}}=O(1) \quad \text { if } \delta \leq \vartheta \leq \pi
$$

We shall need the following interpolation results:

Lemma 15.2. Let $Q$ be a bounded domain in $\mathbb{R}^{2}$ with $C^{2}$ boundary. Then, for any function $v$ in $H^{3}(Q)$,

$$
\sup _{Q}|v| \leq C\left(\int_{Q}|v|^{2}\right)^{1 / 2}+C\left(\int_{Q}|\nabla v|^{2}\right)^{\alpha / 2}\left(\int_{Q}\left(|\nabla v|^{2}+\left|\nabla^{3} v\right|^{2}\right)\right)^{\beta / 2}
$$

and for any function $v$ in $H^{2}(Q)$

$$
\sup _{Q}|v| \leq C\left(\int_{Q}|v|^{2}\right){ }^{1 / 2}+C\left(\int_{Q}|v|^{2}\right) \alpha / 2\left(\int_{Q}\left(|v|^{2}+\left|\nabla^{2} v\right|^{2}\right)\right)^{\beta / 2}
$$

for any $\frac{1}{2}<\beta<1, \alpha=1-\beta$, where $C$ is a constant depending only on $Q, \beta$.

Remark 15.1. The lemma holds also if $Q$ is a rectangle (and therefore also if $Q$ is a finite union of rectangles) since $v$ can then be extended as an $H^{3}$ (or $H^{2}$ ) function into a smooth domain which contains $Q$, without increasing the $H^{3}$ (or $H^{2}$ ) norm by more than a multiplicative constant.

Proof. By Sobolev's inequality

$$
\sup _{Q}|v| \leq C\left(\int_{Q}|v|^{2}\right)^{1 / 2}+C\left(\int_{Q}|\nabla v|^{p}\right)^{1 / p} \quad \forall p>2 .
$$

If we now use the interpolation inequalities [4, Chap. 1 , Sec. 9]

$$
\left(\int_{Q}|\nabla v|^{p}\right)^{1 / p} \leq C\left(\int_{Q}|v|^{2}\right)^{\alpha / 2}\left(\int_{Q}\left(|v|^{2}+\left|\nabla^{2} v\right|^{2}\right)\right)^{\beta / 2}
$$

and

$$
\left(\int_{Q}|\nabla v|^{p}\right)^{1 / p} \leq C\left(\int_{Q}|\nabla v|\right)^{\alpha / 2}\left(\int_{Q}|\nabla v|^{2}+\left|\nabla^{3} v\right|^{2}\right)^{\beta / 2}
$$

where $\beta=1-1 / p$ to estimate the right-hand side, then the inequalities (15.5), (15.6) follow.

Set $\widetilde{W}(x, y)=W(r x, r y)$ for any small $r$. If we rewrite $(14.55),(14.56)$ for $\widetilde{W}$ and then apply (15.5) to $v=\nabla \widetilde{W}$ we obtain, after going back to $\nabla W$,

$$
\sup _{S_{r}^{(1)}}|\nabla W| \leq \frac{C c^{*}}{|z| r^{5 / 2}}\left(r^{2}|z|\right)^{\beta} .
$$


In the same way, by (14.55) and (15.6),

$$
\sup _{S_{r}^{(1)}}\left|\nabla^{2} W\right| \leq \frac{C c^{*}}{r^{3 / 2}} \frac{1}{\left(r^{2}|z|\right)^{1-\beta}} .
$$

Similarly, if we set $\widetilde{W}(x, y)=W\left(\rho_{k n} x, \rho_{k n} y\right)$, where $\rho_{k n}$ is the diameter of $Q_{k n}$, and use (14.57), we obtain (after using also the relations $\rho_{k n} \leq C r, \rho_{k n} \leq C d_{k n}$ )

$$
\begin{gathered}
\sup _{Q_{k n}}|\nabla W| \leq \frac{C c^{*}}{|z| r^{3 / 2} d_{k n}}\left(r^{2}|z|\right)^{\beta}, \\
\sup _{Q_{k n}}\left|\nabla^{2} W\right| \leq \frac{C c^{*}}{r^{1 / 2} d_{k n}} \frac{1}{\left(r^{2}|z|\right)^{1-\beta}} .
\end{gathered}
$$

We now proceed to estimate $\widetilde{f}_{j}$ and $f_{j}$ for $j=1, \ldots, 11$.

From the definitions of $g(R, z)$ and $\|Q\|_{X}$ and the estimate (14.20) we see that if $r \gg|z|^{-1 / 2}$ then, in average,

$$
|\mu| \leq \frac{C}{|z|^{\epsilon / 2}} \frac{r^{\frac{1}{2}-\epsilon}}{r^{4}} \text { in } S_{r}^{(1)}
$$

and

$$
|\mu| \leq \frac{C}{|z|^{\epsilon / 2}} \frac{r^{\frac{1}{2}-\epsilon}}{r^{3} d_{k n}} \quad \text { in } Q_{k n} \quad\left(\bigcup_{n} Q_{k n}=S_{r}^{(2)}\right),
$$

where $S_{r}^{(i)}=S_{k}^{(i)}$ with $R_{k} \approx r$. Since $|h(\vartheta, r)| \leq C \vartheta, d_{k n} \sim y$ and $\vartheta \sim y / r$ if $x>0$, we get

$$
\left|k_{1}\right| \leq \frac{C}{|z|^{\epsilon / 2}} \frac{r^{\frac{1}{2}-\epsilon}}{r^{3}}
$$

in $r$-average, i.e., averaging on intervals of length $r / 2$ about $r$. Hence

$$
\left|\tilde{f}_{1}\right| \leq C r^{-3 / 2} \int_{r}^{r_{0}} s^{5 / 2} \frac{d s}{|z|^{\epsilon / 2} s^{\frac{7}{2}+\epsilon}} \leq \frac{C}{r^{3 / 2}} \frac{1}{\left(|z|^{1 / 2} r\right)^{\epsilon}} .
$$

The same estimate is similarly derived for $f_{1}$. Similarly we also establish the bound

$$
\frac{C}{r^{3 / 2}} \frac{1}{\left(|z|^{1 / 2} r\right)^{1+\epsilon}}
$$

for $\widetilde{f}_{2}$ and $f_{2}$.

Next, by interpolation and (14.55),

$$
\begin{aligned}
\left(f_{J_{r}}\left|D_{x}^{3} F\right|^{2}\right)^{1 / 2} & \leq C\left(f_{J_{r}}\left|D_{x}^{7 / 2} F\right|^{2}\right)^{1 / 4}\left(f_{J_{r}}\left|D_{x}^{5 / 2} F\right|^{2}\right)^{1 / 4} \\
& \leq \frac{C c^{*}}{r^{5 / 2}|z|^{1 / 2}} .
\end{aligned}
$$

Hence for any $r<r_{i}<r_{i+1}<r_{0}$

$$
f_{r_{i}}^{r_{i+1}}\left|D_{x}^{3} F\right|=\left(f_{r_{i}}^{r_{i+1}}\left|D_{x} F\right|^{2}\right)^{1 / 2} \leq \frac{C c^{*}}{r_{i}^{7 / 2}|z|^{1 / 2}}\left(r_{i+1}-r_{i}\right) .
$$


Using this estimate, we deduce that

$$
\begin{aligned}
\left|\tilde{f}_{3}\right| & \leq C r^{-3 / 2} \int_{r}^{r_{0}} s^{3 / 2}\left|D_{x}^{3} F\right| d s \leq C r^{-3 / 2} c^{*} \sum_{r<s_{i}<r_{0}} \frac{s_{i+1}-s_{i}}{s_{i}^{2}|z|^{1 / 2}} \\
& \leq \frac{C c^{*}}{r^{3 / 2}} \frac{1}{r|z|^{1 / 2}} .
\end{aligned}
$$

The same bound holds for $f_{4}$.

$\tilde{f}_{5}$ and $f_{5}$ are estimated in the same way, using the fact that

$$
r^{3 / 2}|z||W(r, \vartheta)| \leq C c^{*} .
$$

To estimate $\widetilde{f}_{6}$ we use integration by parts:

$$
\begin{aligned}
\widetilde{f}_{6} & =C_{0} r^{-3 / 2} \int_{r}^{r_{0}} \frac{s^{5 / 2}}{s} \frac{\partial^{3} W(s, \pi)}{\partial s^{2} \partial y} d s \\
& =C_{0} r^{-2 / 3}\left[s^{3 / 2} \frac{\partial^{2} W}{\partial s \partial y}\right]_{r}^{r_{0}}-\frac{3}{2} C_{0} r^{-3 / 2} \int_{r}^{r_{0}} s^{1 / 2} \frac{\partial^{2} W(s, \pi)}{\partial s \partial y} \equiv I_{1}-I_{2} .
\end{aligned}
$$

By (15.8),

$$
\left|I_{1}\right| \leq C r^{-3 / 2} \frac{r^{3 / 2} c^{*}}{r^{3 / 2}\left(r^{2}|z|\right)^{1-\beta}}=\frac{C c^{*}}{r^{3 / 2}\left(r^{2}|z|\right)^{1-\beta}} .
$$

We can also estimate $I_{2}$ by the same bound, so that

$$
\left|\widetilde{f}_{6}\right| \leq \frac{C c^{*}}{r^{3 / 2}\left(r^{2}|z|\right)^{1-\beta}} .
$$

Similarly we can estimate $f_{6}$ by the same bound.

To estimate $\widetilde{f}_{7}$, recall that

$$
k_{7}=-\int_{0}^{\pi} Q_{2}(r, \vartheta) d \vartheta, \quad Q_{2}=2 \frac{\partial \Delta W}{\partial r} \frac{\partial h}{\partial r}+\Delta W \cdot \mathcal{L}(h) .
$$

We begin with the term corresponding to $2 \frac{\partial \Delta W}{\partial r} \frac{\partial h}{\partial r}$. Thus we have to estimate

$$
\begin{aligned}
& \frac{2}{3} r^{-3 / 2} \int_{r}^{r_{0}} d s \int_{0}^{\pi} s^{5 / 2} \frac{\partial \Delta W}{\partial s} \frac{\partial h}{\partial s} d \vartheta \\
& =\frac{2}{3} r^{-3 / 2}\left[\int_{0}^{\pi} s^{5 / 2} \Delta W \frac{\partial h}{\partial s} d \vartheta\right]_{r}^{r_{0}}-\frac{2}{3} r^{-3 / 2} \int_{r}^{r_{0}} d s \int_{0}^{\pi} \Delta W \frac{\partial}{\partial s}\left(s^{5 / 2} \frac{\partial h}{\partial s}\right) d \vartheta \\
& \equiv J_{1}-J_{2} .
\end{aligned}
$$

We first evaluate the parts $J_{i}^{\prime}$ of $J_{i}$ where the $\vartheta$-integration is over the interval $0<\vartheta<\frac{\pi}{2}$; the points $(s, \vartheta)$ then belong to the domains $S_{k}^{(2)}$, so that $(15.10)$ can 
be used in each $\vartheta$-arc $\Lambda_{k n}$ which belongs to $Q_{k n}$. Using (15.3) we obtain

$$
\begin{aligned}
\left|J_{1}^{\prime}\right| & \leq \frac{C c^{*}}{r^{3 / 2}} \sum \int_{\Lambda_{k n}} \frac{r^{5 / 2}}{r^{1 / 2} d_{k n}} \frac{1}{\left(r^{2}|z|\right)^{1-\beta}} \frac{\epsilon}{r} d \vartheta \\
& =\frac{C c^{*}}{r^{3 / 2}} \frac{r \epsilon}{\left(r^{2}|z|\right)^{1-\beta}} \sum \int_{\Lambda_{k n}} \frac{d \vartheta}{y},
\end{aligned}
$$

where $d_{k n} \approx y$ and $y$ varies in the interval $|z|^{-1 / 2} \leq y \leq r$. Substituting

$$
\vartheta=\frac{y}{r}, \quad d \vartheta=\frac{d y}{r},
$$

we find that

$$
\sum \int_{\Lambda_{k n}} \frac{d \vartheta}{y}=\frac{1}{r} \int_{|z|^{-1 / 2}}^{r} \frac{d y}{y}=\frac{1}{r} \log \left(r|z|^{1 / 2}\right)
$$

and, since $\epsilon=\left(r|z|^{1 / 2}\right)^{-1} \ll 1$,

$$
\left|J_{1}^{\prime}\right| \leq \frac{C c^{*}}{r^{3 / 2}\left(r^{2}|z|\right)^{1-\beta}} .
$$

Next, by (15.3)

$$
\left|\frac{\partial}{\partial s}\left(s^{5 / 2} \frac{\partial h}{\partial s}\right)\right| \leq C s^{1 / 2} \epsilon
$$

Hence, by (15.10),

$$
\begin{aligned}
\left|J_{2}^{\prime}\right| & \leq \frac{C}{r^{3 / 2}} \int_{r}^{r_{0}} d s \sum \int_{\Lambda_{k n}}|\Delta W| s^{1 / 2} \epsilon d \vartheta \\
& \leq \frac{C c^{*}}{r^{3 / 2}} \int_{r}^{r_{0}} \frac{s^{1 / 2} \epsilon}{s^{1 / 2}\left(s^{2}|z|\right)^{1-\beta}} d s \sum \int_{\Lambda_{k n}} \frac{d \vartheta}{d_{k n}} \quad\left(d_{k n} \approx y, \epsilon=\left(s|z|^{1 / 2}\right)^{-1}\right) \\
& \leq \frac{C c^{*}}{r^{3 / 2}} \int_{r}^{r_{0}} \frac{d s}{\left(s^{2}|z|\right)^{1-\beta} s},
\end{aligned}
$$

where (15.13) and the fact that $\epsilon \ll 1$ were used. It follows that

$$
\left|J_{2}^{\prime}\right| \leq \frac{C c^{*}}{r^{3 / 2}\left(r^{2}|z|\right)^{1-\beta}} .
$$

The same bounds can be obtained (more simply) for the interval $\frac{\pi}{2}<\vartheta<\pi$ by using (15.8) instead of (15.10).

Finally, to estimate the term in $\tilde{f}_{7}$ which comes from $\Delta W \cdot \mathcal{L}(h)$ (see $(15.11)$ ), we proceed essentially as in the derivation of the estimate of $J_{2}$.

$f_{7}$ is estimated in a similar way.

We now turn to $\widetilde{f}_{8}, f_{8}$, recalling that

$$
k_{8}=z Q_{1}=\mathcal{L}(h) \cdot z W+2 \frac{\partial h}{\partial r} z \frac{\partial W}{\partial r} .
$$


Since

$$
|\mathcal{L}(h)| \leq \frac{C \epsilon}{s^{2}}=\frac{C}{s^{3}|z|^{1 / 2}},
$$

the contribution to $\widetilde{f}_{8}$ coming from $\mathcal{L}(h) \cdot z W$ is bounded by

$$
C r^{-3 / 2} \int_{r}^{r_{0}} \frac{s^{5 / 2}}{s^{3}|z|^{1 / 2}} \frac{c^{*}}{s^{3 / 2}} d s=\frac{C c^{*}}{r^{3 / 2}\left(r^{2}|z|\right)^{1 / 2}} .
$$

The contribution coming from $2 \partial_{r} h \cdot z \partial_{r} W$ is evaluated by first using integration by parts:

$$
\begin{aligned}
r^{-3 / 2} & \int_{r}^{r_{0}} \int_{0}^{\pi} s^{5 / 2} \frac{\partial h}{\partial s} z \frac{\partial W}{\partial s} d \vartheta d s \\
= & r^{-3 / 2} \int_{0}^{\pi}\left[s^{5 / 2} \frac{\partial h}{\partial s} z W\right]_{r}^{r_{0}} d \vartheta-r^{3 / 2} \int_{r}^{r_{0}} \int_{0}^{\pi} \frac{\partial}{\partial s}\left(s^{5 / 2} \frac{\partial h}{\partial s}\right) z W d \vartheta d s \equiv L_{1}-L_{2} .
\end{aligned}
$$

Since $|\partial h / \partial s| \leq C \epsilon / s$,

$$
\left|L_{1}\right| \leq C \epsilon \frac{c^{*}}{r^{3 / 2}}=\frac{C c^{*}}{r^{3 / 2}} \frac{1}{r|z|^{1 / 2}} .
$$

By (15.14) we also have

$$
\begin{aligned}
\left|L_{2}\right| & \leq C r^{-3 / 2} c^{*} \int_{r}^{r_{0}} \frac{s^{1 / 2} \epsilon}{s^{3 / 2}} d s \quad\left(\epsilon=\left(s|z|^{1 / 2}\right)^{-1}\right) \\
& \leq \frac{C c^{*}}{r^{3 / 2}} \frac{1}{r|z|^{1 / 2}} .
\end{aligned}
$$

Thus, altogether,

$$
\left|\widetilde{f}_{8}\right| \leq \frac{C c^{*}}{r^{3 / 2}} \frac{1}{r|z|^{1 / 2}} .
$$

The same bound is similarly derived for $f_{8}$.

To estimate $\widetilde{f}_{9}$ and $f_{9}$ we first use integration by parts to write

$$
k_{9}=-\frac{1}{r^{2}} \int_{0}^{\pi} \frac{\partial}{\partial \vartheta} \mathcal{L}(W) \cdot \frac{\partial h}{\partial \vartheta} d \vartheta
$$

Then, by integration by parts in $s$,

$$
\begin{aligned}
\widetilde{f}_{9} & =-\frac{1}{3} r^{-3 / 2}\left[\int_{0}^{\pi}\left(\frac{s^{5 / 2}}{s^{2}} \frac{1}{s}\right)\left(s \frac{\partial^{2} W}{\partial s \partial \vartheta}\right) \frac{\partial h}{\partial \vartheta} d \vartheta\right]_{r}^{r_{0}} \\
& +\frac{1}{3} r^{-3 / 2} \int_{r}^{r_{0}} d s \int_{0}^{\pi} d \vartheta \frac{\partial}{\partial s}\left(s^{-1 / 2} \frac{\partial h}{\partial \vartheta}\right) \cdot\left(s \frac{\partial^{2} W}{\partial s \partial \vartheta}\right) \equiv-M_{1}+M_{2} .
\end{aligned}
$$


We first estimate the parts $M_{i}^{\prime}$ of $M_{i}$ where the $\vartheta$-integration is over the interval $0<\vartheta<\frac{\pi}{2}$. Since

$$
\left|s \frac{\partial^{2} W}{\partial s \partial \vartheta}\right| \leq s^{2}\left|\nabla^{2} W\right|
$$

using (15.10) we obtain

$$
\left|M_{1}^{\prime}\right| \leq \frac{C c_{*}}{r^{1 / 2}} \frac{1}{\left(r^{2}|z|\right)^{1-\beta}} \sum \int_{\Lambda_{k n}} \frac{1}{d_{k n}}\left|\frac{\partial h}{\partial \vartheta}\right| d \vartheta .
$$

Noting that $d_{k n} \approx y, \partial h / \partial \vartheta=O(1)$ and substituting $\vartheta=y / r, d \theta=(d y) / r$, we find, upon using (15.13), that the last integral is bounded by $C r^{-1} \log \left(r|z|^{1 / 2}\right)$. Consequently,

$$
\left|M_{1}^{\prime}\right| \leq \frac{C c^{*}}{r^{3 / 2}} \frac{1}{\left(r^{2}|z|\right)^{1-\beta^{\prime}}} \quad \text { for any } \beta<\beta^{\prime}<1 .
$$

Next, using (15.3) and (15.10) we get

$$
\left|M_{2}^{\prime}\right| \leq \frac{C}{r^{3 / 2}} \int_{r}^{r_{0}} \frac{1}{s^{3 / 2}} \frac{s^{2} c^{*}}{s^{1 / 2}\left(s^{2}|z|\right)^{1-\beta}} d s \sum \int_{\Lambda_{k n}} \frac{d \vartheta}{d_{k n}} .
$$

By (15.13), the last integral is bounded by

$$
\frac{1}{s} \log \left(s|z|^{1 / 2}\right)
$$

and therefore

$$
\left|M_{2}^{\prime}\right| \leq \frac{C c^{*}}{r^{3 / 2}} \int_{r}^{r_{0}} \frac{d s}{s\left(s^{2}|z|\right)^{1-\beta^{\prime}}} \leq \frac{C c^{*}}{r^{3 / 2}} \frac{1}{\left(r^{2}|z|\right)^{1-\beta^{\prime}}}
$$

for any $\beta<\beta^{\prime}<1$.

The same estimates can be obtained (more simply) for the interval $\frac{\pi}{2}<\vartheta<\pi$, and so

$$
\left|\widetilde{f}_{9}\right| \leq \frac{C c^{*}}{r^{3 / 2}} \frac{1}{\left(r^{2}|z|\right)^{1-\beta^{\prime}}} .
$$

Similarly we can estimate $f_{9}$ by the same bound.

To estimate $\widetilde{f}_{10}$ we note that

$$
\begin{aligned}
k_{10}=\mathcal{L}^{2}(\varphi) & =\frac{1}{r} \frac{\partial}{\partial r}\left(r \frac{\partial}{\partial r} \mathcal{L}(\varphi)\right) \\
& =\int_{0}^{\pi} \frac{\partial^{4} W}{\partial r^{4}} h(\vartheta) d \vartheta+\sum_{j=0}^{3} \int_{j=0}^{\pi} \frac{\partial^{j} W}{\partial r^{j}} h_{j}(\vartheta, r) d \vartheta \\
& \equiv K+\sum_{j=0}^{3} K_{j},
\end{aligned}
$$

where $h_{j}$ are products of $r$-derivatives of $h$. It will be enough to estimate the term $\widetilde{f}_{10}$ corresponding to $K$; the terms corresponding to the $K_{j}$ can be estimated in the same way (or a bit more simply in the case of $K_{0}, K_{1}$, and $K_{2}$ ). 
By integration by parts we can write

$$
\begin{aligned}
\widetilde{f}_{10} & =\frac{1}{3} r^{-3 / 2} \int_{r}^{r_{0}} d s \int_{0}^{\pi} s^{5 / 2} \frac{\partial^{4} W}{\partial s^{4}} h d \vartheta=\frac{1}{3} r^{-3 / 2}\left[\int_{0}^{\pi} s^{5 / 2} h \frac{\partial^{3} W}{\partial s^{3}} d \vartheta\right]_{r}^{r_{0}} \\
& -\frac{1}{3} r^{-3 / 2} \int_{r}^{r_{0}} d s \int_{0}^{\pi} \frac{\partial}{\partial s}\left(s^{5 / 2} h\right) \cdot \frac{\partial^{3} W}{\partial s^{3}} d \vartheta \equiv N_{1}-N_{2} .
\end{aligned}
$$

We proceed to estimate the terms $N_{i}^{\prime}$ where the $\vartheta$-integration is over $0<\vartheta<\frac{\pi}{2}$. By interpolation [9, p. 49]

$$
\left(f_{Q_{k n}}\left|\nabla^{7 / 2} W\right|^{2}\right)^{1 / 2} \leq C\left(f_{Q_{k n}}\left|\nabla^{2} W\right|^{2}\right)^{1 / 8}\left(f_{Q_{k n}}\left|\nabla^{4} W\right|^{2}\right)^{3 / 8} .
$$

Using (14.57) we get

$$
\left(f_{Q_{k n}}\left|\nabla^{7 / 2} W\right|^{2}\right)^{1 / 2} \leq \frac{C c^{*}}{|z|^{1 / 4} r d_{k n}^{5 / 2}}
$$

The same bound can be similarly established for $\left.\left(f_{Q_{k n}}\right)\left|\nabla^{3} W\right|^{2}\right)^{1 / 2}$.

Hence, if $\ell_{k n}$ is any circular arc or a line segment in $Q_{k n}$ such that

$$
\text { length of } \ell_{k n} \equiv\left|\ell_{k n}\right| \geq c_{0} \rho_{k n},
$$

where $\rho_{k n}$ is the diameter of $Q_{k n}$ and $c_{0}$ is a positive constant, then, by the trace theorem,

$$
\left(f_{\ell_{k n}}\left|\nabla^{3} W\right|^{2}\right)^{1 / 2} \leq \frac{C c^{*} \rho_{k n}^{1 / 2}}{|z|^{1 / 4} r d_{k n}^{5 / 2}} \leq \frac{C c^{*}}{|z|^{1 / 4} r d_{k n}^{2}} \quad\left(\text { since } \rho_{k n} \leq C d_{k n}\right) .
$$

It follows that

$$
f_{\Lambda_{k n}}\left|\nabla^{3} W\right| d \vartheta \leq C\left(f_{\Lambda_{k n}}\left|\nabla^{3} W\right|^{2}\right)^{1 / 2} \leq \frac{C c^{*}}{|z|^{1 / 4} r d_{k n}^{2}},
$$

and, since $|h(\vartheta, r)| \leq C \vartheta$,

$$
\left|N_{1}^{\prime}\right| \leq \frac{C c^{*}}{|z|^{1 / 4}} \sum \int_{\Lambda_{k n}} \frac{\vartheta d \vartheta}{d_{k n}^{2}} .
$$

Substituting $d_{k n} \approx y, \vartheta=y / r, d \vartheta=(d y) / r$, we get

$$
\left|N_{1}^{\prime}\right| \leq \frac{C c^{*}}{r^{3 / 2}} \frac{1}{\left(r|z|^{1 / 2}\right)^{1 / 2}}\left|\log \left(r|z|^{1 / 2}\right)\right| \leq \frac{C c^{*}}{r^{3 / 2}} \frac{1}{\left(r|z|^{1 / 2}\right)^{\frac{1}{2}-\gamma}}
$$

for any small $\gamma>0$. Similarly, since

$$
\left|\frac{\partial}{\partial s}\left(s^{5 / 2} h(\vartheta, s)\right)\right| \leq C s^{3 / 2} \vartheta
$$


we get

$$
\begin{aligned}
\left|N_{2}^{\prime}\right| & \leq \frac{C c^{*}}{r^{3 / 2}} \int_{r}^{r_{0}} \frac{s^{3 / 2}}{|z|^{1 / 4} s}\left[\sum \int_{\Lambda_{k n}} \frac{\vartheta d \vartheta}{d_{k n}^{2}}\right] d s \\
& \leq \frac{C c^{*}}{r^{3 / 2}|z|^{1 / 4}} \int_{r}^{r_{0}} \frac{s^{3 / 2}}{s^{3}}\left|\log \left(s|z|^{1 / 2}\right)\right| d s=\frac{C c^{*}}{r^{3 / 2}} \frac{1}{\left(r|z|^{1 / 2}\right)^{\frac{1}{2}-\gamma}}
\end{aligned}
$$

for any small $\gamma>0$. The parts of $N_{1}, N_{2}$ with $\frac{\pi}{2}<\vartheta<\pi$ can be estimated, more simply, using (14.55) instead of (14.57). We conclude that

$$
\left|\widetilde{f}_{10}\right| \leq \frac{C c^{*}}{r^{3 / 2}} \frac{1}{\left(r|z|^{1 / 2}\right)^{\frac{1}{2}-\gamma}}
$$

and the same bound can be obtained for all the other terms that make up $\tilde{f}_{10}$. Similarly one can estimate $f_{10}$.

We next consider $\widetilde{f}_{11}, f_{11}$, recalling that

$$
k_{11}=\mathcal{L}\left(S_{1}(r)\right), S_{1}(r) \text { as in (14.38). }
$$

The terms corresponding to $\mathcal{L}(W(r, \pi))$ can be estimated as in the case of $k_{6}$. The term corresponding to

$$
\mathcal{L}\left(\int_{0}^{\pi} W(r, \theta) h_{\vartheta \vartheta} d \vartheta\right)
$$

can be estimated quite easily using pointwise estimates on $W, \partial_{r} W$ and $\partial_{r}^{2} W$, noting that we have good bounds on the $r$-derivatives of $h$. All the estimates thus obtained are of the form (14.59).

Completion of the proof of Lemma 6.1. Having proved Lemma 14.1, the proof of Lemma 14.6 is now complete. Thus $A=0$, and it remains to show that

$$
B \equiv 0 \text {. }
$$

Here we shall require the estimate

$$
\left\|Q_{m}\right\|_{X} \ll \frac{C}{\left|z_{m}\right|^{\frac{\epsilon}{2}+\frac{1}{2}}|\log | z_{m}||} .
$$

The proof of (15.16) is similar to that of Lemma 14.6, so we shall only describe it briefly.

First we need to replace Lemma 14.7 by the following lemma.

Lemma 15.3. Suppose

$$
\left|W_{m}\right| \leq \frac{C}{\left|z_{m}\right| r^{1 / 2}} \text { if } \frac{M}{\left|z_{m}\right|^{1 / 2}} \leq r \leq 1
$$

for some positive constants $M, C$. Then, for any constants $N_{2}>N_{1}>0$,

$$
\left|W_{m}\right|=o\left(\frac{1}{\left|z_{m}\right| r^{1 / 2}}\right) \text { for } \frac{N_{1}}{\left|z_{m}\right|^{1 / 2}} \leq r \leq \frac{N_{2}}{\left|z_{m}\right|^{1 / 2}}
$$

as $m \rightarrow \infty$. 
Proof. The proof is based on the following estimate (cf. (14.25)):

$$
\|(W, F)\|_{R} \leq \frac{C}{|z|^{\frac{\epsilon}{2}+\frac{1}{2}}} \text { for all } R<\frac{M}{|z|^{1 / 2}} .
$$

We establish this bound by contradiction: If (15.20) is not true then (with the same notation as in the proof of (14.25)) we derive the inequalities

$$
|\widetilde{W}| \leq \frac{C}{|z|^{1-\frac{\epsilon}{2}-\frac{1}{2}} r^{1 / 2}} \quad(\text { cf. }(14.29))
$$

and

$$
\left|\widetilde{W}_{m}(x, y)\right| \leq \frac{C\left|z_{m}\right|^{\frac{1}{4}+\frac{\epsilon}{2}}}{r^{1 / 2}} \text { if } 1<r<M_{m}\left|z_{m}\right|^{1 / 2}
$$

Also

$$
\left|\widetilde{W}_{m}(x, y)\right| \leq C\left|z_{m}\right|^{\frac{1}{4}+\frac{\epsilon}{2}} r^{\frac{1}{2}+\epsilon} \text { if } 0<r<1,
$$

and we then conclude that the sequence $\hat{W}_{m} /\left|z_{m}\right|^{\frac{1}{4}+\frac{\epsilon}{2}}$ converges to a function $\varphi$ which is identically zero by $[7$, Theorem 1.1$]$. We can then proceed to derive a contradiction by using the same argument as in Lemma 14.7.

Having proved (15.20) we deduce, by the Poincaré inequality, that the functions

$$
\widetilde{W}_{m}(x, y)=W_{m}\left(\rho_{m} x, \rho_{m} y\right) \quad\left(\rho_{m}=\frac{1}{\left|z_{m}\right|^{1 / 2}}\right)
$$

satisfy

$$
\left|\widetilde{W}_{m}(x, y)\right| \leq C\left|z_{m}\right|^{-\frac{3}{4}} r^{\frac{1}{2}+\epsilon} \quad \text { if } r<M
$$

On the other hand, (15.18) yields

$$
\left|\widetilde{W}_{m}(x, y)\right| \leq C\left|z_{m}\right|^{-\frac{3}{4}} r^{\frac{1}{2}-\epsilon} \quad \text { if } M \leq r \leq M\left|z_{m}\right|^{1 / 2},
$$

and these two estimates lead (by means of [7, Theorem 1.1]) to the assertion (15.19).

Next we establish an analogue of Lemma 14.9:

If (14.17) and (15.17) hold, then

$$
\sup _{M\left|z_{m}\right|^{-1 / 2} \leq r \leq 1}\left[\left|z_{m}\right| r^{1 / 2}\left|W_{m}\right|\right] \rightarrow 0 \quad \text { if } m \rightarrow \infty .
$$

Once this is proved, the assertion (15.16) follows.

Proof of (15.21). We define the function $h$ a little differently than before, namely, $h$ satisfies the differential equation

$$
\epsilon^{2} h_{\theta \vartheta \vartheta \vartheta}=h_{\theta \vartheta}+\frac{1}{2} h, \quad 0<\vartheta<\pi,
$$

and the boundary condition (14.36).

Analogously to (14.50) we define

$$
c^{*}=\sup _{M /|z|^{1 / 2} \leq r \leq 1} \sup _{0 \leq \vartheta \leq \pi}\left[|z| r^{1 / 2}|W(r, \theta)|+|z| r^{1 / 2}|F(r)|\right] .
$$


Assuming $B \neq 0$ we deduce that $c^{*} \equiv c_{m}^{*}$ stays away from 0 , uniformly in $m$. We proceed to derive analogously to (14.55) and (14.56) the estimates

$$
\begin{aligned}
|z| R^{\frac{1}{2}+1}\left(f_{S_{R}^{(1)}}|\nabla W|^{2}\right)^{1 / 2} \leq C c^{*}, \\
|z| R^{\frac{1}{2}+2}\left(f_{S_{R}^{(1)}}\left|\nabla^{2} W\right|^{2}\right)^{1 / 2} \leq C c^{*}, \\
R^{\frac{1}{2}+2}\left(f_{S_{R}^{(1)}}\left|\nabla^{4} W\right|^{2}\right)^{1 / 2} \leq C c^{*}, \\
R^{\frac{1}{2}+\frac{3}{2}}\left(f_{J_{R}}\left|D_{x}^{7 / 2} F\right|^{2}\right)^{1 / 2} \leq C c^{*}, \\
|z| R^{\frac{1}{2}+\frac{3}{2}}\left(f_{J_{R}}\left|D_{x}^{5 / 2} F\right|^{2}\right)^{1 / 2} \leq C c^{*},
\end{aligned}
$$

and, analogously to (14.57), the estimates

$$
\begin{aligned}
& |z| R^{\frac{1}{2}+\frac{1}{2}}\left(f_{Q_{k n}}|\nabla W|^{2}\right)^{1 / 2} \leq C c^{*}, \\
& |z| R^{\frac{1}{2}+1} d_{k n}\left(f_{Q_{k n}}\left|\nabla^{2} W\right|^{2}\right)^{1 / 2} \leq C c^{*}, \\
& R^{\frac{1}{2}-1} d_{k n}^{3}\left(f_{Q_{k n}}\left|\nabla^{4} W\right|^{2}\right)^{1 / 2} \leq C c^{*} .
\end{aligned}
$$

The relations (14.43), (14.44) remain the same with one minor change:

Consequently,

$$
\frac{9}{4 r^{2}} \varphi \text { is replaced by } \frac{1}{4 r^{2}} \varphi \text {. }
$$

$$
f_{r r}+\frac{1}{r} f_{r}-\frac{1}{4 r^{2}} f=k(r)
$$

with the same $k(r)$ as before. We can represent $f$ in the form

$$
f(r)=\frac{1}{2} r^{1 / 2} \int_{r_{0}}^{r} s^{1 / 2} k(s) d s-\frac{1}{2} r^{-1 / 2} \int_{r_{0}}^{r} s^{3 / 2} k(s) d s+a r^{-1 / 2}+b r^{1 / 2},
$$

where $a \equiv a_{m}, b \equiv b_{m}$ are uniformly bounded constants, and $a_{m}$ stays uniformly away from zero (as $m \rightarrow \infty$ ). 
The proof of Lemma 14.9 can now be extended (using (15.23), (15.24)) to conclude that (14.59) holds with

$$
\frac{C c^{*}}{r^{3 / 2}} \text { replaced by } \frac{C c^{*}}{r^{1 / 2}},
$$

and this leads to a contradiction, as before, which completes the proof of (15.21).

Proof of Lemma 8.1. Lemma 6.1 deals with the case $R_{k_{m}} \approx 1$, whereas Lemma 8.1 deals with the case

$$
R_{k_{m}} \rightarrow 0, \text { while } R_{k_{m}}\left|z_{m}\right|^{1 / 2} \gg 1 .
$$

If we rescale by introducing

$$
W_{m}^{*}(x, y)=W\left(R_{m} x, R_{m} y\right), \quad F_{m}^{*}(x)=F_{m}\left(R_{m} x\right),
$$

then the proof of Lemma 6.1 can be applied with minor changes.

Remark 15.2. One can also prove Lemma 5.1 without the factor $\log |z|$ : Indeed, we may proceed as before except that we shall now have a weaker estimate on $|\nabla W|$ in (14.57) and (15.24), namely,

$$
\frac{|z| R^{\alpha}}{\left|\log d_{k n}\right|}\left(f_{Q_{k n}}|\nabla W|^{2}\right)^{1 / 2} \leq C c^{*},
$$

where $\alpha=\frac{3}{2}$ and $\alpha=\frac{1}{2}$ respectively. This weaker estimate, however, does not change any of the subsequent estimates. As a consequence, Lemma 11.2 and the existence results in Sections 12,13 also remain valid when the factor $\log (|z|+1)$ is dropped.

\section{ACKNOWLEDGEMENT}

The first author is partially supported by National Science Foundation DMS \# 94-01251. The second author is partially supported by DGICYT Grant PB-0438 and by the Institute for Mathematics and its Applications.

\section{REFERENCES}

[1] S. Agmon, A. Douglis and L. Nirenberg, Estimates near the boundary for solutions of elliptic partial differential equations satisfying general boundary conditions, J. Comm. Pure App. Math., 12 (1959), 623-727. MR 23:A2610

[2] S. Agmon, A. Douglis and L. Nirenberg, Estimates near the boundary for solutions of elliptic partial differential equations satisfying general boundary conditions, II, Comm. Pure Appl. Math., 17 (1964), 35-92. MR 28:5252

[3] G. Duvaut and J.L. Lions, Les inéquations en méchanique et en physique, Dunod, Paris, 1972. MR 57:4778

[4] A. Friedman, Partial differential equations, Holt, Rinehart and Winston, New York 1969. MR 31:6062

[5] A. Friedman and J.J.L. Velázquez, The analysis of coating flows near the contact line, J. Diff. Eqs., 119 (1995), 137-208. MR 96b:35168

[6] A. Friedman and J.J.L. Velázquez, The analysis of coating flows in a strip, J. Diff. Eqs., 121 (1995), 134-182. MR 96i:76032

[7] A. Friedman and J.J.L. Velazquez, Liouville type theorems for fourth order elliptic equations in a half plane, Trans. Amer. Math. Soc., 349 (1997), 2537-2603. 
[8] P.D. Lax, A Phragmén-Lindelöf theorem in harmonic analysis and its application to some questions in the theory of elliptic equations, Comm. Pure Appl. Math., 10 (1957), 361-389. MR 20:229

[9] J.J. Lions and E. Magenes, Problèmes aux limites non homogénes et applications, vol. 1, Dunod, Paris, 1968. MR 40:512

Institute for Mathematics and its Applications, University of Minnesota, MinneapoLIS, MinNESOTA 55455

Departamento de Matematica Aplicada, Universidad Complutense, Facultad de Matematicas, 28040 Madrid, Spain 